\title{
Prosodic Features for a Maximum Entropy Language Model
}

\author{
Oscar Chan \\ This thesis is presented for the degree of \\ Doctor of Philosophy \\ of \\ The University of Western Australia
}

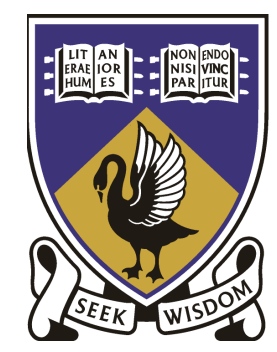

School of Electrical, Electronic and Computer Engineering

The University of Western Australia

July 2008 


\section{Abstract}

A statistical language model attempts to characterise the patterns present in a natural language as a probability distribution defined over word sequences. Typically, they are trained using word co-occurrence statistics from a large sample of text. In some language modelling applications, such as automatic speech recognition (ASR), the availability of acoustic data provides an additional source of knowledge. This contains, amongst other things, the melodic and rhythmic aspects of speech referred to as prosody. Although prosody has been found to be an important factor in human speech recognition, its use in ASR has been limited.

The goal of this research is to investigate how prosodic information can be employed to improve the language modelling component of a continuous speech recognition system. Because prosodic features are largely suprasegmental, operating over units larger than the phonetic segment, the language model is an appropriate place to incorporate such information. The prosodic features and standard language model features are combined under the maximum entropy framework, which provides an elegant solution to modelling information obtained from multiple, differing knowledge sources. We derive features for the model based on perceptually transcribed Tones and Break Indices (ToBI) labels, and analyse their contribution to the word recognition task.

While ToBI has a solid foundation in linguistic theory, the need for human transcribers conflicts with the statistical model's requirement for a large quantity of training data. We therefore also examine the applicability of features which can be automatically extracted from the speech signal. We develop representations of an utterance's prosodic context using fundamental frequency, energy and duration features, which can be directly incorporated into the model without the need for manual labelling. Dimensionality reduction techniques are also explored with the aim of reducing the computational costs associated with training a maximum entropy model. Experiments on a prosodically transcribed corpus show that small but statistically significant reductions to perplexity and word error rates can be obtained by using both manually transcribed and automatically extracted features. 


\section{Acknowledgements}

First of all, I would like to express my deepest gratitude to my supervisor, Roberto Togneri. It was he who first sparked my interest in speech and language processing. His expert guidance and advice, as well as his encouragement during the more difficult times, have been invaluable. This work would not have been possible without him.

Special thanks is due to John Henderson, for those few but insightful discussions on prosody. His ideas and suggestions have been very helpful, and were a major factor in the formulation of this thesis. I am grateful to Ramachandran Chandrasekhar, for generously providing his LaTeX thesis templates. Thanks also go to the staff members in the School of Electrical, Electronic and Computer Engineering, especially Linda.

I wish to extend my appreciation to my friends and colleagues in CIIPS - Aik Ming, Marco, Serajul, Daniel and Weiqun. It has been a great pleasure working together with them, and the many stimulating discussions we have had, technical or otherwise, have made this experience a very enjoyable and rewarding one.

I would also like to acknowledge the Australian Postgraduate Awards programme and the Australasian Speech Science Technology Association for providing financial assistance during my candidature.

Finally, I wish to thank my family and friends for all the support they have provided over the years. 


\section{Contents}

Abstract iii

Acknowledgements $\quad$ v

Contents $\quad$ X

$\begin{array}{ll}\text { List of Figures } & \text { xii }\end{array}$

\begin{tabular}{l|l} 
List of Tables & xiv \\
\hline
\end{tabular}

1 Introduction $\quad 1$

1.1 Research Objectives . . . . . . . . . . . . . . . 2

1.2 Document Overview ............................ 3

2 Language Modelling for ASR 5

2.1 Language Modelling . . . . . . . . . . . . . . . 6

2.1.1 N-gram models . . . . . . . . . . . . . . . . 7

2.1.2 Smoothing . . . . . . . . . . . . . . . 7

2.1.3 $N$-gram extensions and other models . . . . . . . . . . . 11

2.1.4 Decision trees . . . . . . . . . . . . . . . . . 15

2.2 Automatic Speech Recognition . . . . . . . . . . . . . 16

2.2.1 Feature extraction . . . . . . . . . . . . 17

2.2 .2 Acoustic modelling . . . . . . . . . . . . . . . . . . 18

2.3 Decoding . . . . . . . . . . . . . . . . . . . . . 20

2.3.1 Viterbi decoding . . . . . . . . . . . . . 21

2.3.2 Stack decoding . . . . . . . . . . . . . . . . . 22

2.3 .3 Multi-pass decoding . . . . . . . . . . . . . . . 23

2.4 Evaluation . . . . . . . . . . . . . . . . . . 26

2.4.1 ASR evaluation . . . . . . . . . . . . . . 26

2.4.2 Statistical significance . . . . . . . . . . . . . 27 


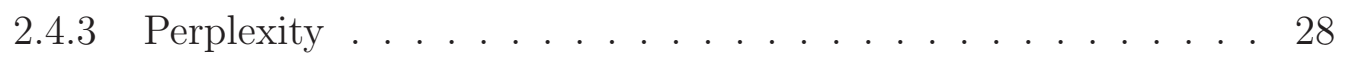

3 Prosody 31

3.1 Prosodic Features . . . . . . . . . . . . . . . . . 32

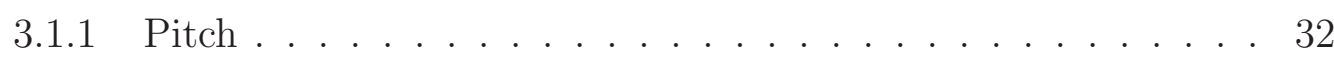

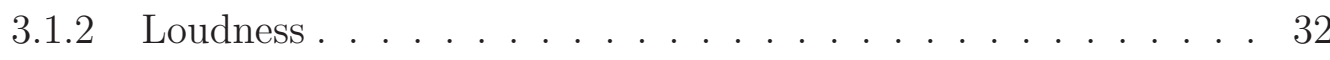

3.1 .3 Length . . . . . . . . . . . . . . . . . 33

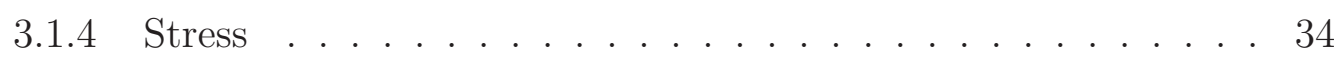

3.1.5 Tone ............................ 34

3.1 .6 Intonation . . . . . . . . . . . . . . . . 34

3.2 Functions of Prosody . . . . . . . . . . . . . . . . . 34

3.2.1 Roles in human communication . . . . . . . . . . . . 35

3.2.2 Cross-linguistic differences . . . . . . . . . . . . . . 39

3.3 Modelling Prosody . . . . . . . . . . . . . . . . . . 40

3.3.1 Pierrehumbert's model ............... . . . 41

3.3.2 Tones and Break Indices . . . . . . . . . . . . . . . . . . 42

3.3.3 Alternate modelling approaches ... . . . . . . . . 46

3.4 Prosody in ASR . . . . . . . . . . . . . . . . . 47

3.5 Prosodic Knowledge for Language Modelling . . . . . . . . . . . . . . 52

4 Maximum Entropy $\quad 55$

4.1 Maximum Entropy Principle . . . . . . . . . . . . . . . . 55

4.2 Maximum Entropy Models . . . . . . . . . . . . . . . . 57 57

4.2.1 Representing knowledge ............... . . 57

4.2 .2 Conditional models . . . . . . . . . . . . . . . 5 57

4.2 .3 Minimum divergence . . . . . . . . . . . . . 60

4.3 Parameter Estimation _. . . . . . . . . . . . . . . . 61

4.3.1 Iterative scaling . . . . . . . . . . . . . . 61 61

4.3.2 Gradient based methods . . . . . . . . . . . . . 63

4.4 Smoothing . . . . . . . . . . . . . . . . 64 64

4.4 .1 Feature selection . . . . . . . . . . . . . . 64

4.4 Regularisation . . . . . . . . . . . . . . 65

4.5 Combining Knowledge Sources . . . . . . . . . . . . . . . . . 68

5 Language Modelling with ToBI Features $\quad 71$

5.1 Corpus . . . . . . . . . . . . . . . . . 72

5.2 Evaluating Prosodic Features . . . . . . . . . . . . . 74

5.3 Language Modelling . . . . . . . . . . . . . . . . 78 


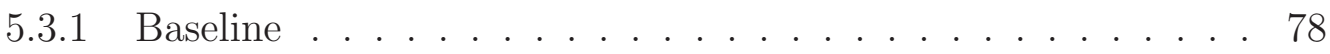

5.3 .2 ToBI features . . . . . . . . . . . . . . . . 78

5.3 .3 Perplexity results . . . . . . . . . . . . . . . 80

5.4 Speech Recognition . . . . . . . . . . . . . . . . . 83

5.4.1 Multi-pass decoding . . . . . . . . . . . . . . 84

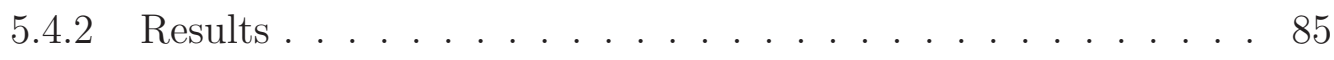

5.5 Conclusion . . . . . . . . . . . . . . . . . . 90

6 Automatic Feature Extraction $\quad 91$

6.1 Aims . . . . . . . . . . . . . . . . . . . 92

6.2 Prosodic Feature Extraction . . . . . . . . . . . . . . . 92

6.2.1 Duration . . . . . . . . . . . . . . . 93

6.2.2 Fundamental frequency . . . . . . . . . . . . . . 98

6.2 .3 Energy . . . . . . . . . . . . . . . . . 101

6.3 Feature Analysis . . . . . . . . . . . . . . . . . 102

6.3 .1 Duration . . . . . . . . . . . . . . . . 102

6.3.2 F0 and RMS energy . . . . . . . . . . . . . . 104

6.4 Summary . . . . . . . . . . . . . . . . . . . . . . . 108

7 Language Modelling with Raw Features $\quad 111$

7.1 Handling Non-Binary Features . . . . . . . . . . . . . . . . . . . . 112

7.1.1 Real-valued features . . . . . . . . . . . . . . . 112

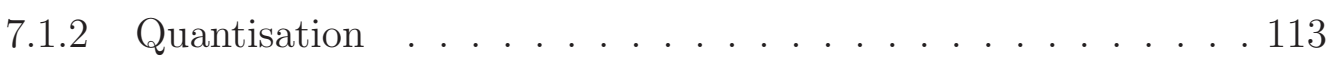

7.2 Dimensionality Reduction . . . . . . . . . . . . . . . 114

7.2.1 Linear dimensionality deduction . . . . . . . . . . . . . 114

7.2.2 Locality Preserving Projections . . . . . . . . . . . . . . 116

7.3 Prosody Labelling . . . . . . . . . . . . . . . . . . . . . . . . . 117

7.3.1 Baseline model . . . . . . . . . . . . . . . . . . 118

7.3.2 Prosodic model features . . . . . . . . . . . . . . . 118

7.3.3 Results . . . . . . . . . . . . . . . . . 119

7.3.4 Language modelling results . . . . . . . . . . . . . . . . 121

7.4 Direct Modelling . . . . . . . . . . . . . . . . . . . . . 122

7.4.1 Motivation . . . . . . . . . . . . . . 122

7.4.2 Word clustering in prosody space . . . . . . . . . . . . 123

7.4.3 Modelling prosodic history . . . . . . . . . . . 126

7.4.4 Results . . . . . . . . . . . . . . . . . 127

7.5 Noise Robustness . . . . . . . . . . . . . . . . . . . . . 130

7.5.1 Sources of noise . . . . . . . . . . . . . . 131 


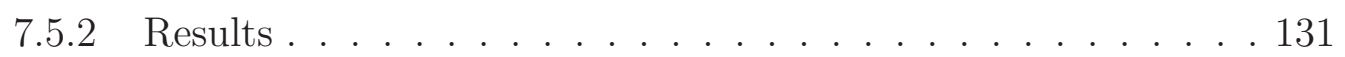

7.6 Conclusion . . . . . . . . . . . . . . . . . . . 134

\begin{tabular}{ll|l}
8 & Conclusion & 137 \\
\hline
\end{tabular}

8.1 Summary . . . . . . . . . . . . . . . . . . . . 137

8.2 Future Research . . . . . . . . . . . . . . . . . . . . . . . . 139

8.2.1 Alternate Corpus . . . . . . . . . . . . . . . . . . 140

8.2 .2 Improved Prosody Modelling . . . . . . . . . . . . . . . . . . 140

8.2 .3 System Integration . . . . . . . . . . . . . . . . . . 140

$\begin{array}{ll}\text { A Penn Treebank Tag Set } & 143\end{array}$

\begin{tabular}{l|l} 
Bibliography & 160 \\
\hline
\end{tabular} 


\section{List of Figures}

2.1 Back-off paths of a factored language model. . . . . . . . . . . . . . . 13

2.2 Structure of a speech recogniser. . . . . . . . . . . . . . . . 17

2.3 HMM generation of an observation sequence. . . . . . . . . . . . 19

2.4 Trellis for of Viterbi decoding. . . . . . . . . . . . . . . . 21

2.5 Generation of hypotheses for multi-pass decoding. . . . . . . . . . . 24

2.6 An $N$-best list. . . . . . . . . . . . . . . . . . . . . . 25

2.7 A fragment of a word hypothesis graph. . . . . . . . . . 26

3.1 Finite-state grammar of allowable tone sequences. . . . . . . . . . . 41

3.2 A ToBI transcription of an utterance. . . . . . . . . . . . . . . 45

5.1 Distribution of pitch accents and break indices in the training partition of the RNC data. . . . . . . . . . . . . . . . . . . 73

5.2 Oracle accuracies of $N$-best lists for $1 \leq N \leq 100$. . . . . . . . . . 85

5.3 Histogram of the rank of the oracle hypothesis before and after reranking by LM-ABS. . . . . . . . . . . . . . . . . . . . . . . . . 89

6.1 Speaker-dependent duration PDFs for the word state. . . . . . . . 96

6.2 Post-processing of an F0 contour. . . . . . . . . . . . . . . . . 99

6.3 F0 features derived from the automatically extracted pitch contour. . 100

6.4 Distribution of normalised word durations for the four prosodic classes.102

6.5 Distribution of normalised durations for accented phrase-final words, separated by POS. . . . . . . . . . . . . . . . . 103

6.6 A typical rise-fall F0 pattern occurring during the word reaches, and the (dotted) least squares regression line fitted over the region. . . . . 106

7.1 A language model rescoring system using intermediate labelling. . . . 121

7.2 A language model rescoring system using direct prosodic features. . . 124

7.3 Projections of word cluster centroids onto a 3-dimensional LPP space. 126

7.4 Modelling of two types of noise sources in the speech signal. . . . . . 131 
7.5 F0 contours extracted from signals corrupted with additive noise at

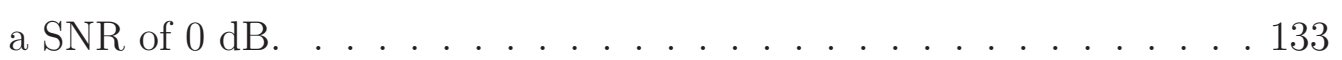

7.6 F0 contour extracted from a signal in the presence of reverberation with a $\mathrm{RT}_{60}$ of $0.5 \mathrm{~s} . \ldots \ldots \ldots$. . . . . . . . . . . . . . . . . . . . . 


\section{List of Tables}

3.1 The relationships between the acoustic and perceptual realisations of prosody, and their major linguistic functions. . . . . . . . . . . . . 39

5.1 Mutual information between a word and its features over the training set. . . . . . . . . . . . . . . . . . . . . . . . . .

5.2 Perplexities of language models using features comprising length $m$ sequences of ToBI labels. . . . . . . . . . . . . . . . . 81

5.3 Distribution of pitch accents and break indices in the training set. . . 83

5.4 Recognition accuracies of language models using ToBI features. . . . 86

5.5 Misrecognitions of three systems according to error type. . . . . . . . 88

6.1 Average KL distances between word duration distributions for three different normalisation schemes. . . . . . . . . . . . . . . 95

6.2 Means and standard deviations (in brackets) of z-score normalised phone durations for the three phonemes in the word state. . . . . . . 97

6.3 Means (and standard deviations) of raw and normalised word durations for each of the four prosodic classes. . . . . . . . . . . . . 102

6.4 Means (and standard deviations) of voicing, F0 and RMS energy features for the four combinations of pitch accent and break index. . . 105

6.5 Means (and standard deviations) of quantised F0 and RMS energy features for the four combinations of pitch accent and break index. . . 107

6.6 Relationship between prosodic events and automatically extracted features. Entries for which a feature is useful for determining the prosodic class are marked as either $\mathrm{H}$ (indicating the mean feature value is higher than average) or L (lower than average). . . . . . . . . 109

7.1 Pitch accent and break index classification accuracies using prosodic features and LPP dimensionality reduction. . . . . . . . . . . . . . 119

7.2 Cosine similarities between words and cluster centroids. . . . . . . . 125 
7.3 Perplexities and word recognition accuracies of models using cosine similarity features. . . . . . . . . . . . . . . . . . . . 128

7.4 Pitch accent and break index classification accuracies using prosodic features and LPP dimensionality reduction. . . . . . . . . . . . 130

7.5 Word accuracies of LM-D8 when using prosodic features extracted from data corrupted by additive noise. . . . . . . . . . . . . . . . 132

7.6 Word accuracies of LM-D8 when using prosodic features extracted from data corrupted by reverberant noise. . . . . . . . . . . . . . 134 


\section{Chapter 1}

\section{Introduction}

Spoken languages contain a wide range of information. While speech is used primarily to convey a sequence of words, the speech signal contains more than just the acoustic properties defining the phonemic segments. There is paralinguistic information which includes speaker-specific information such as their gender, are and emotions. There is also prosody, which bears linguistic content pertaining to the message being conveyed. The term prosody is broadly used to refer to the melodic and rhythmic aspects of speech, but there is less agreement on a more precise definition. Cutler et al. [1] identifies a continuum of interpretations. At one end, prosody is defined as the abstract phonological structure of speech, without consideration of its realisation. At the other extreme, no distinction is made between the phonological structure and its acoustic realisation, and the term prosody has been used synonymously with prosodic features. A more comprehensive definition is also given, which takes into account both the phonetic and phonological aspects of prosody: "the linguistic structure which determines the suprasegmental properties of utterances". Prosodic features are considered to be suprasegmental because they operate on domains larger than the phonemic segment. These range from the smallest domain of the syllable, up through words, phrases, utterances and paragraphs, to the largest domain of the discourse. Unlike speech segments, which can largely be characterised in terms of absolute acoustic parameters, prosodic features tend to be meaningful only with reference to a time series $[2,3]$. Prosodic features are also inherent in speech segments, and these are sometimes included in the definition of prosody as microprosodic features.

The complex nature of prosody has been responsible, at least in part, for its lack of use in automatic speech processing. Despite being an important aspect of human-to-human communication, its precise functions are difficult to quantify and model. Automatic speech processing systems tend to have a modular design, with 
each component being responsible for a specific task. In contrast, prosody has a multitude of functions including, but not limited to, conveying emotion, guiding dialogue structure [4], resolving ambiguity [5], demarcating word and phrase boundaries [6], and in some languages, distinguishing between phonetically similar sounds. All these functions are realised as variations in the acoustic parameters of fundamental frequency, energy and duration, and this makes if difficult, if not impossible, to isolate the phonological factors governing their changes.

There is also some disparity between the traditional lines of prosody research and the requirements of speech processing systems. While there is a large body of research covering many aspects of prosody, much of it is not directly applicable to automated systems. These systems, taking advantage of recent advances in computing power, simply treat the speech processing task as a more general statistical pattern matching problem. Inputs to such systems are typically statistics gathered by observing a large data sample. To be effective, these statistics need to be both general and reliable. Highly specific knowledge about prosodic behaviour, or knowledge that is situational, therefore tends to be of lesser value to an automatic speech processing system.

Despite these difficulties, though, there are many potential uses for prosody in speech systems. At the lowest level, prosody can help word recognition [7] either directly, or by aiding correct segmentation of continuous speech. The close relationship between prosodic and syntactic structure [8] makes it a a good candidate for use in keyword spotting and speech understanding applications. Correct prosody is vital aspect of natural sounding speech [9], and a speech synthesis system must take this into account. The ultimate goal, of course, is to use prosody in all aspects of speech processing - from guiding dialogue choices through to recognising speech and generating responses. While this may be a long-term goal, the topic has garnered substantial interest, and much progress has been made in recent years.

\subsection{Research Objectives}

The goal of this research is to further the understanding of prosody in the context of automatic speech processing. The focus, in particular, is on the use of suprasegmental features to assist in continuous speech recognition. More specifically, the objectives are to:

- Examine how prosody behaves at and above the word level, and how this behaviour is related to word identity and syntax. 
- Investigate prosodic realisations to obtain a set of representative acoustic parameters that are both highly correlated with prosodic events and can be extracted from the speech signal without human supervision.

- Formulate these parameters into features for a statistical language model that capture relationships between prosody and lexical information for the purposes of word prediction.

- Evaluate the models, both in isolation and when coupled to a speech recogniser, and examine the effects of introducing prosodic knowledge into the system.

\subsection{Document Overview}

The remainder of this dissertation is organised as follows. Chapter 2 will provide an introduction to the ASR problem. It will cover the operation of a typical speech recognition system, with particular emphasis on the role of language models in recognising continuous speech. It will also examine some difficulties involved with developing language models, and a number of alternatives to the standard $n$-gram model will be reviewed.

Chapter 3 will present an overview of the prosody of speech. It will outline some of the roles that prosody plays in human-to-human communication, as well as ways in which it has been used in automatic processing. Prosody is perceptual, and this chapter will review some methods of quantifying prosodic information.

The maximum entropy model lies at the core of this research, and it will be discussed in Chapter 4. The chapter will begin with an example demonstrating the principle of maximum entropy, and then present the general form of the model. Some of the advantages and disadvantages of this model will be examined. This chapter will describe how these models can be used for language modelling, and highlight the model's ability to combine diverse sources of knowledge under a consistent framework.

Chapter 5 will present an approach to developing language models using prosodic information. It will make use of of a prosodically transcribed corpus, and an investigation of the relationship between prosody, words and syntax will be performed. Following this, prosodic features for maximum entropy models will be derived, and the models evaluated using both information theoretic criteria and application in a speech recognition system.

In Chapter 6, the issue of automatically extracting prosodic features is considered. Because perceptual annotation of prosody is a difficult and time-consuming 
task, there are substantial benefits to being able to obtain this information in an unsupervised manner. This chapter will examine the relationship between two types of prosodic events, accents and boundaries, and various acoustic parameters. It will focus specifically on the behaviour of these parameters at the word level, as this is the natural unit of the language model.

In Chapter 7, the acoustic parameters obtained from the investigations of Chapter 6 are applied to language modelling tasks. Two approaches are described. The first makes use of some perceptual information to train prosodic labelling models. The second approach does not use any perceptual transcriptions, and trains language models directly on acoustic parameters. The performance of both approaches are examined. Finally, an investigation of the robustness of the prosodic features under a variety of noise conditions is conducted.

A summary of the findings are presented in Chapter 8. A number of further research directions will also be explored here. 


\section{Chapter 2}

\section{Language Modelling for ASR}

A natural language is a language used as a means of communication amongst humans. Unlike formal languages, which adhere to strict rules specifying their syntax, natural languages contain many complexities and ambiguities which make them difficult to define. The goal of language modelling is to develop a good approximation of a natural language by identifying and characterising patterns within the language.

Many natural language processing (NLP) tasks make use of a language modelling component to provide higher level knowledge. Acoustic models used in automatic speech recognition (ASR) systems generally model sub-word units such as phones, and are therefore unable to capture information at the linguistic level. For all but the most highly constrained ASR tasks, a language model providing this additional knowledge is a necessary component of the system. Another task in which language models are used is machine translation. Language structure can vary greatly in both syntax and morphology, and a machine translation system is required to have detailed models of both source and target languages. Yet more applications of language models include information retrieval, optical character recognition, handwriting recognition and others.

This chapter will examine the role of language modelling in the context of automatic speech recognition. It will begin with an overview of the language modelling problem, and introduce the widely used $n$-gram model. Issues pertaining to parameter estimation of $n$-gram models will also be examined in this section. The second section will provide an overview of the speech recognition process, which consists of four distinct tasks: feature extraction, acoustic modelling, language modelling and decoding. These tasks and their inter-operation will be briefly summarised in this section. In the third section, the task of combining acoustic and language models is considered. It will discuss search algorithms used in the decoding process, as well as rescoring techniques used in multi-pass decoding. The final section of 
this chapter will describe evaluation techniques for speech recognisers as well as introduce perplexity, a recogniser-independent metric for evaluating language model performance.

\subsection{Language Modelling}

Like many solutions to NLP tasks, a distinction can be drawn between knowledgebased and data-driven approaches to the statistical language modelling problem. Knowledge-based approaches attempt to directly apply human knowledge of linguistic structure to describe the language. A commonly used, and well known example is the Context Free Grammar, which defines a set of hierarchical rules representing syntactic constraints. The alternative data-driven approaches rely on automatic training of statistical models by observing a sample of data. These models enjoy a number of advantages over knowledge-based models, including:

- Simplicity: Unsupervised or partially supervised training algorithms for statistical language models can greatly decrease the amount of human effort required to develop them.

- Reusability: The training procedures for statistical models are very general, and can be applied to data from a variety of domains.

- Consistency: Learning parameters by observing empirical data ensures the model does not contain inconsistent information. Additionally, automatic training procedures are capable of identifying relevant patterns in the data which may not be readily apparent.

The main disadvantages to statistical models are:

- Data requirements: In order to accurately estimate model parameters, a large number of training samples are required. Creating this data can be costly and time-consuming.

- Limited knowledge representation: The knowledge which can be learnt by a statistical model is inherently restricted by the model's form. For example, a model which applies a Markovian assumption is incapable of modelling dependencies which extend beyond the available context. It can also be difficult to incorporate potentially useful prior knowledge into a statistical framework. 
With the increasing availability of large databases designed for NLP tasks, the data requirements of training statistical language models becomes easier to satisfy. Additionally, significant increases in computing power have allowed the use of sophisticated models which are capable of modelling more complex phenomena.

\subsubsection{N-gram models}

The probability of a length $m$ word sequence $\left(w_{1}, \ldots, w_{m}\right) \equiv w_{1}^{m}$ can be decomposed, via application of the chain rule, to:

$$
p\left(w_{1}^{m}\right)=\prod_{i=1}^{m} p\left(w_{i} \mid w_{1}^{i-1}\right)
$$

The purpose of a language model is to provide an estimate of $p\left(w_{i} \mid w_{1}^{i-1}\right)$. The potential number of parameters in a model of this form is $|V|^{m}$ where $|V|$ is the size of the vocabulary, so calculating such a model would be infeasible even for moderate $|V|$ and $m$. Therefore, some additional constraints are required in order to obtain a practicable model. The approach applied in $n$-gram language modelling is to use a Markov model approximation such that the identity of a word is conditional only on the previous $n-1$ words:

$$
p\left(w_{i} \mid w_{1}^{i-1}\right) \approx p\left(w_{i} \mid w_{i-n+1}^{i-1}\right)
$$

for some fixed $n$. Typical values of $n$ are one, two or three, and these are commonly referred to as unigram, bigram and trigram models respectively.

\subsubsection{Smoothing}

Maximum likelihood (ML) estimates of $n$-gram model probabilities, $p\left(w_{i} \mid w_{i-n+1}^{i-1}\right)$, can be obtained by observing word frequencies in a training corpus:

$$
p\left(w_{i} \mid w_{i-n+1}^{i-1}\right)=\frac{c\left(w_{i-n+1}^{i}\right)}{c\left(w_{i-n+1}^{i-1}\right)}
$$

where $c(\cdot)$ is the count of occurrences of a specified word sequence. In practice, however, it is unlikely that reliable estimates of all model parameters can be obtained from limited data resources. This is the data sparsity problem, and it is a pervasive issue in NLP tasks. Often, many events will not be seen in a given set of training data, and performing the ML estimation of Equation (2.3) will result in these events being assigned zero probability. Consequently probabilities of non-zero count events 
are overestimated, leading to over-fitting of the model.

Smoothing refers to techniques for redistributing a model's probability mass in order to cope with the effects of sparse data. All smoothing schemes employ some form of discounting to redistribute probability mass away from observed events to unobserved events. Some smoothing schemes also make use of lower order probability distributions in combination with discounting to more accurately model rare events. A number of well-established smoothing methods are described below.

\section{Additive smoothing}

The simplest form of smoothing, additive smoothing [10], increases the count of all events, both seen and unseen, by a constant value $k$. ML estimation is then performed using the modified counts, giving:

$$
p\left(w_{i} \mid w_{i-n+1}^{i-1}\right)=\frac{c\left(w_{i-n+1}^{i}\right)+k}{c\left(w_{i-n+1}^{i-1}\right)+k|V|}
$$

The performance of this method of smoothing is poor, as it tends to overestimate the probabilities of unseen events [11].

\section{Good-Turing smoothing}

In Good-Turing smoothing [12], the discount applied to an event is determined by its observed frequency in the training data. More specifically, the probability mass of events occurring with frequency $r+1$ is reallocated to $r$ count events. An event observed $r$ times in a sample of size $n$ will therefore have probability $\frac{r^{*}}{n}$, where the adjusted count, $r^{*}$, is defined as:

$$
r^{*}=(r+1) \frac{E\left(n_{r+1}\right)}{E\left(n_{r}\right)}
$$

$E\left(n_{r}\right)$ is an estimate of the number of events occurring exactly $r$ times and sometimes its empirical value in the training data, $n_{r}$, is used instead. This is a noisy measure, however, and it is quite common to have $n_{r}=0$ for large $r$. Therefore, the distribution of $n_{r}$ itself needs to be smoothed [12,13].

\section{Jelinek-Mercer smoothing}

The discounting techniques described above redistribute probability mass uniformly to unseen events, but this is not necessarily desirable. Intuitively, a common word is more likely to occur for a given context than a rare word, even if both were 
unobserved in the training data. This issue is addressed by Jelinek-Mercer smoothing [14], which interpolates a high order model with lower order models to improve the accuracy of its estimates. The smoothed $n$-gram model is defined as a linear interpolation of an ML-estimated $n$-gram, $p_{\mathrm{ML}}\left(w_{i} \mid w_{i-n+1}^{i-1}\right)$, with a smoothed $(n-1)$ gram $p_{\lambda}\left(w_{i-n+2}^{i-1}\right)$. This recursive definition can be stopped at either a unigram or a uniform model. The distribution of the smoothed $n$-gram model is:

$$
p_{\lambda}\left(w_{i} \mid w_{i-n+1}^{i-1}\right)=\lambda_{w_{i-n+1}^{i-1}} p_{\mathrm{ML}}\left(w_{i} \mid w_{i-n+1}^{i-1}\right)+\left(1-\lambda_{w_{i-n+1}^{i-1}}\right) p_{\lambda}\left(w_{i-n+2}^{i-1}\right)
$$

The interpolation weights, $\lambda_{w_{i-n+1}^{i-1}}$, are dependent on the context and can be estimated using a set of held-out data. Bahl et al. [15] suggested the use of deleted interpolation, in which the data is partitioned into a number of subsets used for training either the model or interpolation weights. These are iteratively rotated similarly to cross-validation, and the resulting weights averaged.

\section{Back-off smoothing}

Like the Jelinek-Mercer method, back-off smoothing uses model interpolation to deal with sparsity. The general form of a back-off model is:

$$
p_{\mathrm{bo}}\left(w_{i} \mid w_{i-n+1}^{i-1}\right)=\left\{\begin{array}{cl}
\alpha\left(w_{i} \mid w_{i-n+1}^{i-1}\right) & \text { if } c\left(w_{i-n+1}^{i}\right)>0 \\
\gamma\left(w_{i-n+1}^{i-1}\right) p_{\mathrm{bo}}\left(w_{i} \mid w_{i-n+2}^{i-1}\right) & \text { if } c\left(w_{i-n+1}^{i}\right)=0
\end{array}\right.
$$

where $\alpha\left(w_{i} \mid w_{i-n+1}^{i-1}\right)$ is a discounted higher order distribution, $p_{\mathrm{bo}}\left(w_{i} \mid w_{i-n+2}^{i-1}\right)$ is a lower order back-off distribution and $\gamma\left(w_{i-n+1}^{i-1}\right)$ is a normalisation term. Back-off models differ from Jelinek-Mercer smoothed models in that interpolation only takes place for zero count events. Otherwise, the discounted ML estimate is used.

A specific instance of the back-off method is Katz smoothing [16]. In this technique, counts of events having a frequency above some threshold $r_{t}$, are unmodified. Counts of the remaining observed events are discounted by a factor $d_{r}$, and this probability mass is redistributed to unseen events according to the distribution of the lower order model. The justification for the thresholding is that the distribution of frequently occurring events in the training data can be considered sufficiently accurate that the original ML estimates should be retained. This gives the following discounted distribution:

$$
\alpha\left(w_{i} \mid w_{i-n+1}^{i-1}\right)=\left\{\begin{array}{cl}
\frac{c\left(w_{i-n+1}^{i}\right)}{c\left(w_{i-n+1}^{i-1}\right)} & \text { if } c\left(w_{i-n+1}^{i}\right)>r_{t} \\
d_{r} \frac{c\left(w_{i-n+1}^{i}\right)}{c\left(w_{i-n+1}^{i-1}\right)} & \text { if } 0<c\left(w_{i-n+1}^{i}\right) \leq r_{t}
\end{array}\right.
$$


In Katz smoothing, $d_{r}$ is chosen such that the discounts are proportional to that of Good-Turing smoothing, resulting in:

$$
d_{r}=\frac{\frac{r^{*}}{r}-\frac{\left(r_{t}+1\right) n_{r_{t}+1}}{n_{1}}}{1-\frac{\left(r_{t}+1\right) n_{r_{t}+1}}{n_{1}}}
$$

Katz smoothing is widely used, and has been found to perform consistently well [17].

\section{Absolute discounting}

In absolute discounting [18], a constant $0 \leq D \leq 1$ is subtracted from the counts of all observed events. This is combined with linear interpolation to give:

$$
p\left(w_{i} \mid w_{i-n+1}^{i-1}\right)=\frac{\max \left\{c\left(w_{i-n+1}^{i}\right)-D, 0\right\}}{\sum_{w_{i}} c\left(w_{i-n+1}^{i}\right)}+\left(1-\lambda_{w_{i-n+1}^{i-1}}\right) p\left(w_{i} \mid w_{i-n+2}^{i-1}\right)
$$

where the weights are chosen to ensure the probabilities sum to one:

$$
1-\lambda_{w_{i-n+1}^{i-1}}=\frac{D}{\sum_{w_{i}} c\left(w_{i-n+1}^{i}\right)} N_{1+}\left(w_{i-n+1}^{i-1} \cdot\right)
$$

$N_{1+}\left(w_{i-n+1}^{i-1} \cdot\right)$ is the number of distinct words appearing after the sequence $w_{i-n+1}^{i-1}$, and the discount value $D$ is usually set to [18]:

$$
D=\frac{n_{1}}{n_{1}+2 n_{2}}
$$

where $n_{1}$ and $n_{2}$ are the number of higher-order $n$-grams observed one and two times respectively. Absolute discounting has been found to provide an accurate estimate of true event counts in testing data [17].

\section{Kneser-Ney smoothing}

An extension of absolute discounting, known as Kneser-Ney smoothing, was proposed in [19]. While it uses back-off interpolation of the form Equation (2.5), it differs from other interpolated smoothing techniques in its construction of the lower order distribution. Rather than being defined analogously to the higher order distribution, it is designed to satisfy the constraint that the marginals of the smoothed higher order distribution equal those observed in the training sample:

$$
\tilde{p}\left(w_{i} \mid w_{i-n+2}^{i-1}\right)=\sum_{w_{i-n+1}} p\left(w_{i} \mid w_{i-n+1}^{i-1}\right) p\left(w_{i-n+1} \mid w_{i-n+2}^{i-1}\right)
$$


with $\tilde{p}\left(w_{i} \mid w_{i-n+2}^{i-1}\right)$ being the empirical distribution. The solution satisfying this constraint is:

$$
p\left(w_{i} \mid w_{i-n+2}^{i-1}\right)=\frac{N_{1+}\left(\cdot w_{i}\right)}{\sum_{w_{i}} N_{1+}\left(\cdot w_{i}\right)}
$$

where $N_{1+}\left(\cdot w_{i}\right)$ is the number of distinct words preceding $w_{i}$. This approach is motivated by the idea that the absence of an $n$-gram event in the higher order distribution may be meaningful, and not necessarily a consequence of sparse data. Kneser and Ney [19] noted that in the Wall Street Journal corpus, the word dollars has a high unigram frequency; however, it only appears in a very limited context, namely after numbers and country names. It is claimed, then, that backing off to a lower order distribution for such words would result in an inappropriately high probability. The construction of the lower order distribution in this fashion has been found to be one of the major factors contributing to Kneser-Ney smoothing's good performance [17].

\section{Modified Kneser-Ney smoothing}

Based on observations from an evaluation of smoothing techniques, Chen and Goodman [17] proposed a modified version of Kneser-Ney smoothing. This variant of Kneser-Ney smoothing uses linear interpolation of the form of Equation (2.4) as opposed to the original back-off interpolation. Additionally, instead of using a single fixed discount $D$ as in Equation (2.6), the value of the discount is dependent on the frequency of the event to be discounted. Specifically, three discount parameters are used: $D_{1}, D_{2}$, and $D_{3+}$ for events with one, two and three or more counts respectively. The values of $D$ can either be optimised using held-out data, or approximated as:

$$
\begin{gathered}
D_{1}=1-2 Y \frac{n_{2}}{n_{1}} \\
D_{2}=2-3 Y \frac{n_{3}}{n_{2}} \\
D_{3+}=3-4 Y \frac{n_{4}}{n_{3}}
\end{gathered}
$$

where $Y=\frac{n_{1}}{n_{1}+2 n_{2}}$. Modified Kneser-Ney smoothing was shown to outperform a number of other smoothing algorithms under a wide range of conditions [17].

\subsection{3 $\quad N$-gram extensions and other models}

\section{Class $N$-gram models}

Traditional $\mathrm{N}$-gram models have seen widespread use because of their speed and simplicity. A major weakness, however, is that they have very little expressive power. Increasing the value of $n$ can improve their ability to model dependencies in 
the data, but this solution is heavily restricted by practical considerations.

Class-based $n$-grams [20] are an extension of standard $n$-gram models which cluster words into equivalence classes and explicitly model both word and class distributions. A class n-gram model has the form:

$$
p\left(w_{i} \mid w_{i-n+1}^{i-1}\right)=p\left(w_{i} \mid C_{i}\right) p\left(C_{i} \mid C_{i-n+1}^{i-1}\right)
$$

where $C_{i}$ is the class to which word $w_{i}$ belongs. Alternative formulations of the class-based model also include [21]:

$$
\begin{aligned}
& p\left(w_{i} \mid w_{i-n+1}^{i-1}\right)=p\left(C_{i} \mid w_{i-n+1}^{i-1}\right) p\left(w_{i} \mid w_{i-n+1}^{i-1} C_{i}\right) \\
& p\left(w_{i} \mid w_{i-n+1}^{i-1}\right)=p\left(w_{i} \mid C_{i-n+1}^{i-1}\right) \\
& p\left(w_{i} \mid w_{i-n+1}^{i-1}\right)=p\left(C_{i} \mid C_{i-n+1}^{i-1}\right) p\left(w_{i} \mid C_{i-n+1}^{i}\right)
\end{aligned}
$$

By modelling class contexts rather than word contexts, the parameter space, and therefore storage and computation requirements, of the model is reduced. Additionally, this lowers the model's susceptibility to data sparsity in comparison to word $n$-grams of the same order. Intuitive support for this approach can also be found. Different words can have similar meaning and these words will often be observed in similar circumstances. For example, it is not unreasonable to expect that the word Monday can appear in the same context as the word Tuesday. Grouping together such similar words can allow a model to more accurately predict an event based on its class membership, even if that event was not observed in training. The performance of this type of model is obviously dependent on the cluster mapping, and finding this is not a trivial task. Clustering words by semantics or syntax is an intuitive choice; however, this does not necessarily perform well in the statistical framework of a language model. More often, the clustering process is guided by information theoretic measures with respect to the data $[20,22]$.

\section{Factored models}

A factored language model represents each word as a vector of $K$ factors, $w_{i} \equiv$ $\left\{f_{i}^{1}, \ldots, f_{i}^{K}\right\}$, where a factor may be any property of a word, such as its stem, semantics, syntactic class or morphological class. The model provides a distribution over factors $p\left(f_{i}^{1: K} \mid f_{i-n+1: i-1}^{1: K}\right)$ much like an $n$-gram. In fact, an $n$-gram model can be considered to be a special case of a factored language model in which the surface form of a word is its only factor, while a class-based model is the equivalent of a factored model with both the word's form and its class as factors. An example of 


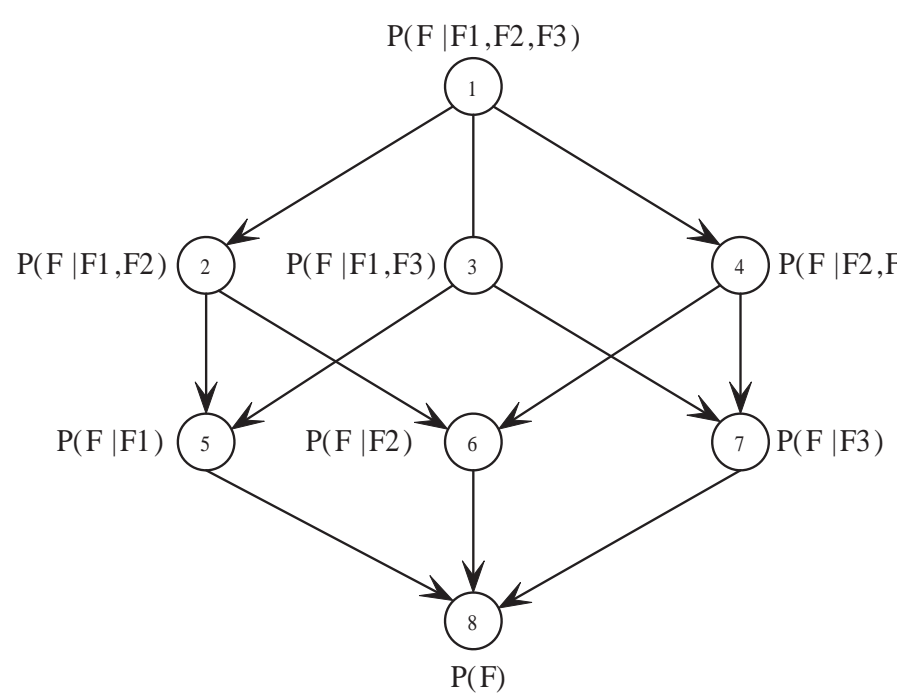

Figure 2.1: Back-off paths of a factored language model.

a factored language model is that of Kirchhoff et al. [23], which used the root, $r_{i}$, pattern, $p_{i}$, and morphological class, $m_{i}$, of word $w_{i}$ as factors in a factored trigram to model the morphologically complex Arabic language:

$$
\begin{aligned}
p\left(w_{i} \mid w_{i-1}, w_{i-2}\right)= & p\left(r_{i}, p_{i}, m_{i} \mid w_{i-1}, w_{i-2}\right) \\
= & p\left(r_{i} \mid p_{i}, m_{i}, w_{i-1}, w_{i-2}\right) \\
& p\left(p_{i} \mid m_{i}, w_{i-1}, w_{i-2}\right) \\
& p\left(m_{i} \mid w_{i-1}, w_{i-2}\right)
\end{aligned}
$$

As with class-based models, the reduction in the space of modelled parameters can lead to greater robustness against sparse data.

Factored language models perform back-off interpolation, and the formulation of the model allows great flexibility in this process. In a standard $n$-gram, the context used by the lower order distribution, as given in Equation (2.5), is simply the original context with the oldest word removed. This is a logical choice, as the predictive power of a word in the context will tend to decrease as its distance from the predicted word increases. In a factored language model, however, there can be multiple factors having the same distance from the target word. In addition, the heterogeneity of the factors means that discarding parent variables based on distance alone may be suboptimal. The set of all potential back-off paths can be described by a directed graph where each node specifies a different context, as shown in Figure 2.1 . 
The choice of back-off path can be decided in advance, or chosen dynamically based on statistical criteria. It is also possible to combine scores of multiple paths using Generalized Parallel Backoff [24]. The back-off equation, assuming two parents, is given by:

$$
p_{\mathrm{GBO}}=\left\{\begin{array}{cl}
\alpha\left(f \mid f_{1}, f_{2}\right) & \text { if } c\left(f, f_{1}, f_{2}\right)>\tau \\
\gamma\left(f_{1}, f_{2}\right) g\left(f, f_{1}, f_{2}\right) & \text { if otherwise }
\end{array}\right.
$$

where $\alpha\left(f \mid f_{1}, f_{2}\right)$ is a ML distribution discounted by any of the standard discounting techniques, $\gamma\left(f_{1}, f_{2}\right)$ is a normalising term and $g\left(f, f_{1}, f_{2}\right)$ is the back-off function. As the name suggests, this is a generalisation of the standard $n$-gram back-off of Equation (2.5), and the two are equivalent when the factors $\left(f, f_{1}, f_{2}\right)$ correspond to words $\left(w_{i}, w_{i-1}, w_{i-2}\right)$, and $g\left(f, f_{1}, f_{2}\right)=p\left(f \mid f_{1}\right)$. The function $g\left(f, f_{1}, f_{2}\right)$ specifies how back-off paths are selected and the way in which probabilities are estimated from these paths. An example $g$ would be to back-off to the node having the highest probability amongst all parent nodes. Alternatives include combining estimates across subsets of parents using mean or weighted mean functions [25]. A factored $n$-gram with $k$ factors per word has $2^{n k}$ subsets of conditioning factors, and a set of $m$ factors describes $m$ ! back-off paths. It is therefore not possible to determine the optimal model structure through an exhaustive search. Instead, genetic algorithms are used to rapidly converge on a solution. While this approach does not necessarily find the optimal solution, it has been demonstrated to outperform a manual search based on linguistic knowledge [26].

\section{Grammatical models}

A fundamental flaw of the $n$-gram modelling paradigm is its incorrect independence assumption. Assuming a word is dependent only on the previous two words, as done by the common trigram model, is obviously incorrect. $\mathrm{N}$-grams are also very knowledge deficient. Although they are used to model natural languages, the model merely treats words as a collection of symbols without giving consideration to the structure of the language. A class of language models which attempt to address these issues are grammatical language models. Grammatical language models attempt to incorporate linguistic knowledge into the model in the form of grammars. These grammars, often with foundations in linguistic research, provide a much richer view of natural languages than that which an $n$-gram can infer from local contextual information.

A simple, well known grammar is the Context Free Grammar (CFG). A CFG consists of a finite set of terminal and non-terminal symbols, and production rules 
governing the expansion of non-terminal symbols. Terminal symbols of the grammar are the words in the vocabulary, and non-terminals represent syntactic constituents. A sentence can then be described using a CFG by repeatedly expanding non-terminal symbols into one or more other terminal or non-terminal symbols, until only terminal symbols remains. This forms a hierarchical, tree-like structure where the leaf nodes of the tree correspond to the words of the sentence. A CFG can be extended to a Probabilistic Context Free Grammar (PCFG), in which a non-terminal can have multiple possible expansions described by a probability distribution. Training of a PCFG is performed using an annotated corpus, or treebank, and the Inside/Outside algorithm [27] is used to learn ML estimates of rule probabilities. While CFGs and PCFGs are most commonly used in parsing tasks, it is noted by Charniak [28] that many parsers compute a joint probability $p(\pi, s)$, where $\pi$ is the parse of sentence $s$. Language model probabilities $p(s)$ can then be calculated by marginalising over all legal parses:

$$
p(s)=\sum_{\pi} p(\pi, s)
$$

PCFGs provide a well structured way of incorporating linguistic knowledge into a language model, and a number of solutions have taken this approach $[29,30,31]$.

Another grammatical formalism which has been used in language modelling is the dependency grammar [32]. Rather than decomposing a sentence into a treelike structure, dependency grammars construct links between words having a headdependent relationship. The inclusion of this type information allows word prediction to be conditioned more intelligently than simply using a fixed length history, and has performance improvements have been demonstrated in speech recognition [33] and information retrieval [34] tasks.

\subsubsection{Decision trees}

A decision tree can be used as a language model [35] by partitioning the histories with a set of binary questions. Each leaf in the tree contains a probability distribution, and each internal node contains one arbitrary binary decision about the history. Trees are constructed by greedily choosing, at each node, the question that maximises some specified criteria about the resulting partitions. Given a complete tree, the probability of a word sequence can be determined by traversing the tree along a path specified by evaluations of the history. The result of the question contained at each node determines which child node to evaluate next, and this is repeated until a leaf node containing a final distribution is reached.

Decision tree models can avoid the very strict Markov assumption of $n$-grams, 
although some limitations on the history and set of potential questions, still need to be enforced in order to reduce computational costs. Another advantage of this approach over $n$-gram modelling is that the number of distributions is dependent on the training data rather than the vocabulary size or the number of classes, and can possibly describe it more accurately. However, the process of constructing the tree can be very computationally intensive.

\section{Maximum entropy models}

Maximum entropy models, also known as exponential or multinomial logistic regression models, were first suggested for use in language modelling in [36]. These models will be discussed in greater detail in Chapter 4.

\subsection{Automatic Speech Recognition}

Given a speech waveform represented by a sequence of observations $O=\left(o_{1}, \ldots, o_{t}\right)$, the task of an automatic speech recognition (ASR) system is to determine the word sequence $W=\left(w_{1}, \ldots, w_{n}\right)$, which is most likely to have generated the input signal. It is often referred to as the noisy channel model, as the speech waveform can be considered to be an encoding of the word sequence which has been passed through a noisy communications channel. The recogniser is then required to recover the original information from the noisy input. This process can be expressed probabilistically as:

$$
\hat{W}=\underset{W}{\arg \max } p(W \mid O)
$$

The space of the acoustic observations is very large, so $p(W \mid O)$ is difficult to estimate. However, Bayes' rule can be applied to obtain a more easily computable form of the expression:

$$
\begin{aligned}
\hat{W} & =\underset{W}{\arg \max } \frac{p(O \mid W) p(W)}{p(O)} \\
& =\underset{W}{\arg \max } p(O \mid W) p(W)
\end{aligned}
$$

The $p(O)$ term on the denominator is fixed for each maximisation, and can therefore be ignored without affecting the result. Equation (2.10) gives the basic problem a speech recogniser is required to solve. The process is typically decomposed into a number of sub-tasks, as depicted by Figure 2.2. 


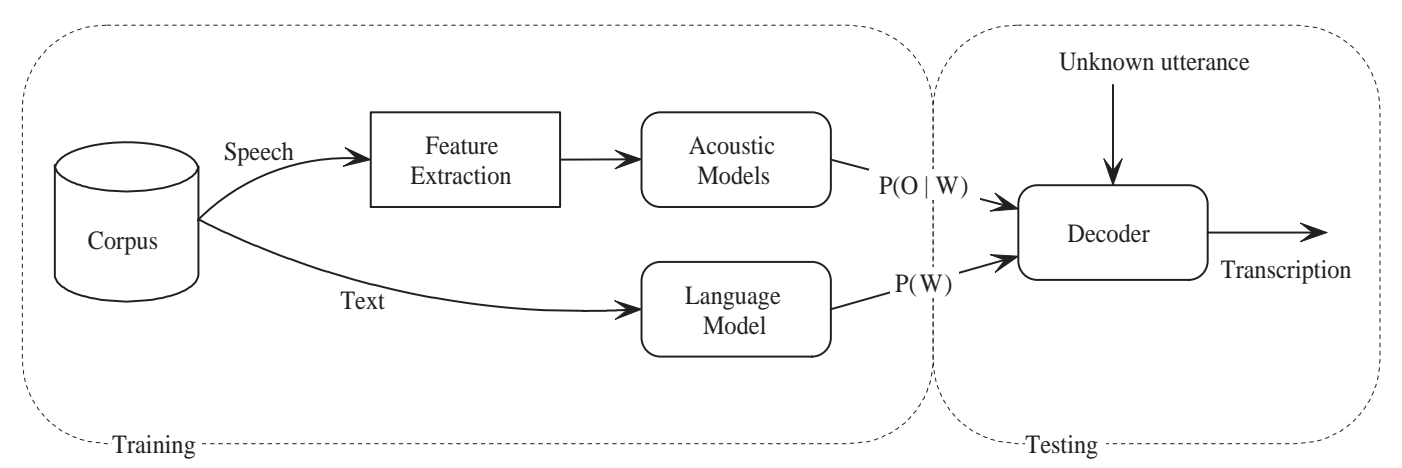

FiguRE 2.2: Structure of a speech recogniser.

The first stage of this process is feature extraction. The goal of this step is to transform the raw speech signal into a representation which is simpler to model and has high discriminatory properties. The transformed speech, represented as a sequence of features $O$, is then used to train acoustic models of linguistic units which provide estimates of $p(O \mid W) . \quad p(W)$ is estimated by the language model, which is trained using text transcriptions of the speech data. Finally, given the observations of an unknown utterance, the decoder searches for the sequence $W$ which maximises $p(O \mid W) p(W)$. Language models have been discussed in section 2.1, and the remaining components are described below.

\subsubsection{Feature extraction}

Speech waveforms exhibit a large amount of variability, the causes of which include phonetic identity, background noise, channel effects, speaker physiology and emotion. Therefore, it is undesirable to use the speech signal directly in a speech recogniser. Instead, feature extraction is performed in order to isolate those characteristics associated with phonetic identity while simultaneously eliminating variability caused by other effects.

The first step of any speech signal analysis is digitisation, which involves sampling the amplitude of the speech waveform at regular intervals using an analog to digital converter. Common sampling rates are $8 \mathrm{kHz}$ and $16 \mathrm{kHz}$. Each sample is stored with a fixed number of bits, so amplitude values are also quantised in this process.

The goal of feature extraction is to derive a set of parameters from the speech signal which describe the envelope of the power spectrum. Speech is considered to be short-time stationary, and the durations of acoustic events such as phones lie in the 10 - 100 ms range. Therefore, analysis of the signal is also done on a comparable time scale. The short-term spectra are calculated, typically every $10 \mathrm{~ms}$, by taking 
the Fourier transform of the signal multiplied by a window function with a width of 20 - 30 ms. A window function with tapered ends, such as the Hamming window, is commonly used.

There are many types of feature representations, such as linear predictive coding (LPC) [37], perceptual linear prediction (PLP) [38], and Mel-Frequency Cepstral Coefficients (MFCCs) [39]. The latter are perhaps the most widely used features in current speech recognition systems. The mel-frequency scale is a perceptually motivated scale which is approximately linear below $1 \mathrm{kHz}$ and logarithmic above 1 $\mathrm{kHz}$ :

$$
\operatorname{Mel}(f)=2595 \log _{10}\left(1+\frac{f}{700}\right)
$$

In MFCC feature extraction, the spectrum is mapped onto the mel-scale by applying a bank of band-pass filters uniformly spaced on the mel-scale. The logarithm of the squared magnitude of the filter-bank output is then taken to perform a range compression similar to that of human hearing. Finally, MFCC parameters are obtained by performing an inverse Fourier transform on the mel log amplitudes. Typically, only the first 12 MFCCs are used.

MFCCs are static features which characterise the spectral properties of a shorttime stationary segment of speech. The performance of a speech recognition can be improved by also including dynamic features representing the changes in the spectrum over time. A common way of computing these features is to take the first and second order time derivatives of the static features.

\subsubsection{Acoustic modelling}

Acoustic models are required to provide the likelihoods $p(O \mid W)$ of Equation (2.10). The high dimensionality of the observation sequence $O$ makes it prohibitive to estimate the distribution directly from the training corpus. The solution that has dominated current speech technology has been the use of the hidden Markov model (HMM) [40,37].

An HMM is a stochastic finite state automaton defined by two stochastic processes: a Markov chain, and an output random variable associated with each state. State changes occur at discrete time intervals, and transition probabilities are stationary. It is also assumed that the output generated on entry to each state is independent of both previous observations and the state history. Because the output of the HMM is probabilistic, the underlying state sequence for a given output sequence is effectively hidden from the observer, giving rise to the model's name. An $\operatorname{HMM} \lambda=(\mathbf{A}, \mathbf{B}, \Pi)$ can be specified by: 


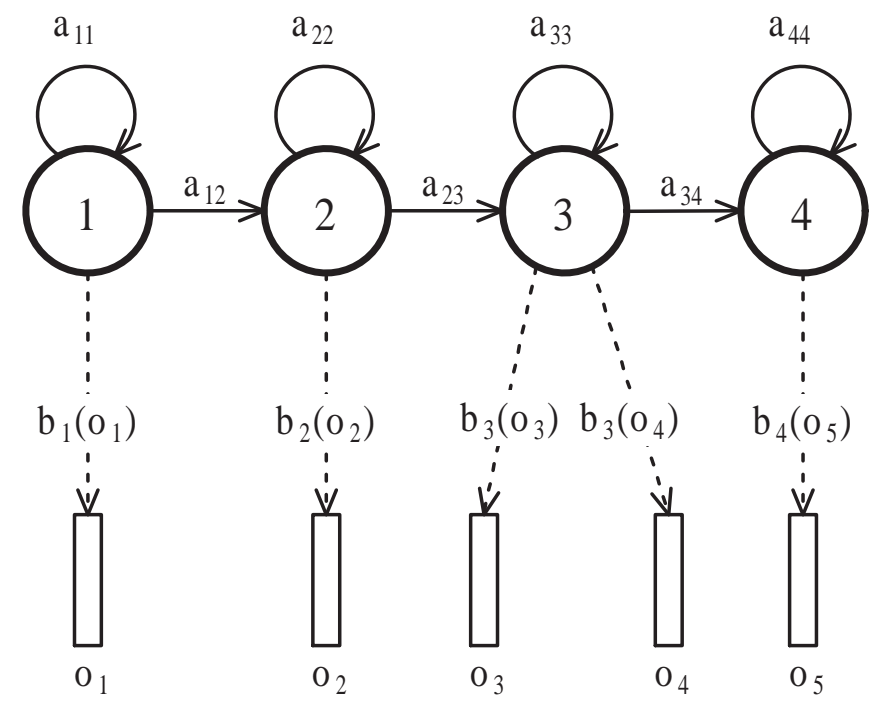

FiguRE 2.3: HMM generation of an observation sequence.

- A set of initial state distributions $\Pi=\left\{\pi_{i}\right\}$, where $\pi_{i}$ is the probability of being in state $i$ at time $t=0$.

- A set of transition probabilities $\mathbf{A}=\left\{a_{i j}\right\}$, where $a_{i j}$ is the probability of transitioning from state $i$ to state $j$.

- A set of output distributions $\mathbf{B}=\left\{b_{i}(o)\right\}$, where $b_{i}(o)$ is the probability of observation $o$ while in state $i$.

An example of a simple HMM is shown in Figure 2.3.

HMMs can be used to model speech as a piecewise stationary process. The possible values of the output distributions lie in the space of acoustic observations, so a sequence of observations $O=\left(o_{1}, \ldots, o_{T}\right)$ can be modelled as a sequence of HMM states $X=\left(x_{1}, \ldots, x_{T}\right)$ that emits $O$. The Markov chain is therefore used to model the temporal characteristics of speech, while the output random variable models the spectral characteristics. For highly constrained tasks, each HMM can be used to model a word, though in more general situations, the sub-word unit of the phone is used. Multiple phone models may then be concatenated to form a composite HMM representation of words or longer sequences.

HMMs for which the output probability distributions $b_{i}$ are defined on a discrete space are referred to as discrete HMMs. The outputs are drawn from a finite alphabet of symbols, with probabilities specified by the state's distribution function. In continuous HMMs, the distributions are defined on continuous spaces. These 
distributions are often constrained to be mixtures of a particular family of distributions, such as Gaussians, which have simple parameterisations. This alleviates some of the added computational costs associated with using continuous distributions. Semicontinuous HMMs [41] have also been proposed as a compromise, using a fixed set of mixture components and state-dependent output probabilities specifying the weights of these components.

Training an HMM involves estimating the parameters of the model given a set of observation sequences. This is done using the Baum-Welch re-estimation procedure [42], an instance of the Expectation Maximization (EM) algorithm [43]. Given an initial set of HMM parameters, the Baum-Welch algorithm iteratively calculates updated parameters and is guaranteed to converge on a locally optimal solution that maximises the likelihood of the training data.

\subsection{Decoding}

For an HMM with known parameters, $\lambda=(\mathbf{A}, \mathbf{B}, \Pi)$, the probability of generating an observation sequence $O$ by traversing through state sequence $X$ is:

$$
p(O, X \mid \lambda)=\prod_{t=1}^{T} a_{x(t) x(t+1)} b_{j}\left(o_{t}\right)
$$

The likelihood of the observation sequence $p(O \mid \lambda)$ can then be calculated by summing over all state sequences:

$$
p(O \mid \lambda)=\sum_{X}\left(\prod_{t=1}^{T} a_{x(t) x(t+1)} b_{j}\left(o_{t}\right)\right)
$$

Often, most of the probability is contained in a single state sequence [44]. Therefore, rather than performing the computationally expensive summation, Equation (2.11) can be approximated using only the most likely sequence:

$$
p(O \mid \lambda)=\max _{X} \prod_{t=1}^{T} a_{x(t) x(t+1)} b_{j}\left(o_{t}\right)
$$

If each HMM represents a single word $w$, then Equation (2.12) can be considered to be equivalent to $p(O \mid w)$. This likelihood, combined with the language model's $a$ priori probability, give the two terms required to solve the recognition problem of Equation (2.10). Since the space of all possible word sequences is very large, a direct computation is infeasible. An alternate solution to Equation (2.10) is to construct 


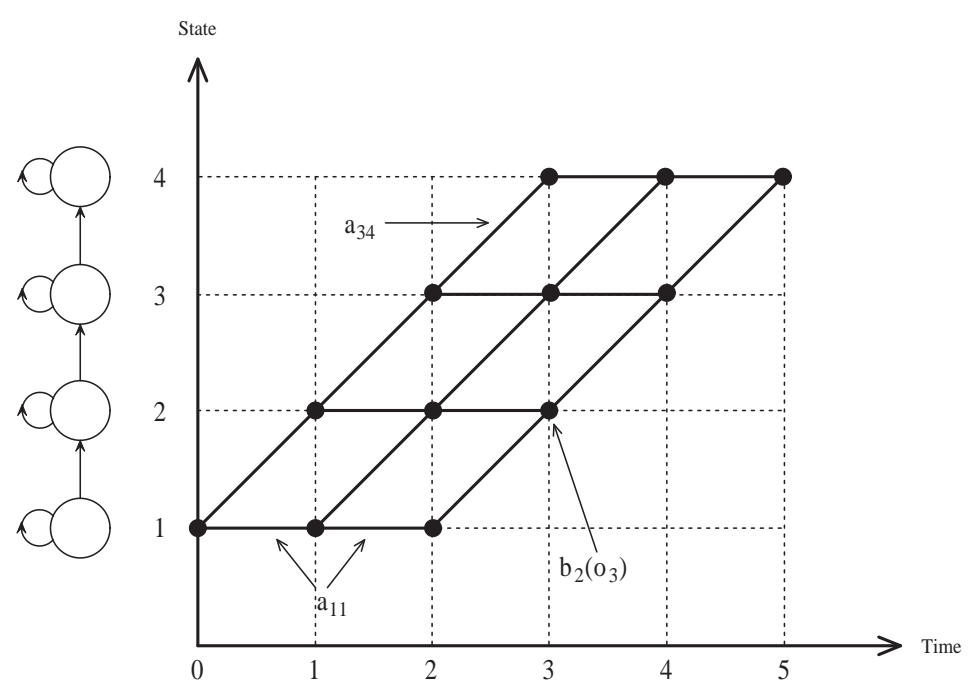

Figure 2.4: Trellis for of Viterbi decoding.

a network of HMMs by connecting word HMMs in sequence. If the transition probabilities between words are governed by a language model, then searching through the resulting composite HMM using Equation (2.12) gives $p(O \mid W) p(W)$.

\subsubsection{Viterbi decoding}

One way of efficiently calculating Equation (2.12) is with the Viterbi algorithm, a dynamic programming algorithm which assumes the optimal path to any intermediate state forms a part of the optimal path to the final state. The globally optimal path can then be calculated recursively as a concatenation of optimal sub-paths. If $\phi_{j}(t)$ is defined as the probability of being in state $j$ at time $t$ after observing sequence $\left(o_{1}, \ldots, o_{t}\right)$, and:

$$
\phi_{j}(1)=\pi_{j} b_{j}\left(o_{t}\right)
$$

then the Viterbi recursion is given by:

$$
\phi_{j}(t)=\max _{i}\left\{\phi_{i}(t-1) a_{i j}\right\} b_{j}\left(o_{t}\right)
$$

for $1<j<N$. The execution of this algorithm can be visualised as a traversal through a 2-dimensional matrix called a trellis, an example of which is shown in Figure 2.4.

The horizontal dimension represents time, and the vertical dimension represents HMM states. The trellis is traversed in a column-wise manner, starting from the left. At each time frame $t$, a new probability is calculated by Equation (2.3.1) using 
model transition probabilities, state-dependent output distributions and values in the adjacent column. This probability is then stored in the trellis node along with the identity of the previous state with the highest probability. The topology of HMMs used for speech recognition is generally constrained to have left-to-right order, so state transitions only occur in one direction. The probability of the optimal path is then:

$$
\phi_{N}(T)=\max _{i}\left\{\phi_{i}(T) a_{i N}\right\}
$$

and the state sequence can be recovered by tracing back through the states stored at each time frame.

If a network of HMMs is constructed from a set of individual word or phone models, and transitions between these models are determined by a bigram language model, then the Viterbi algorithm can be used to find the most likely sequence of words given an observation sequence. The size of the composite model, and therefore the search space, can become very large for large vocabularies. So, in practice, not all paths are expanded at each time frame. Instead, only those paths which have probabilities within some percentage of the most probable path are considered. A Viterbi search with this pruning technique is often referred to as beam search [45].

\subsubsection{Stack decoding}

The time-synchronous operation of the Viterbi decoder can be considered to be a breadth first search. An alternative decoding strategy is stack decoding [46], which performs best first search. Instead of extending all paths at each time, a best first search extends only the most likely path in an asynchronous manner.

The likelihood of a path will tend to decrease with increasing length, and the stack decoder needs to be able to compare paths of differing lengths. Therefore, the best path is not necessarily the one with the highest partial likelihood, but the one which is expected to have the highest total likelihood. The estimated total log likelihood $h^{*}(p)$ of partial path $p$ consists of two components. $f(p(t))$ is the exact log likelihood of $p$, and is analogous to $\phi_{j}(t)$ in Viterbi decoding. $g^{*}(p)$ is an estimate of the $\log$ likelihood of the remainder of $p$ if it were to be extended to completion. The estimated total log likelihood is then:

$$
h^{*}(p)=f(p)+g^{*}(p)
$$

The stack is used to maintain a list of partial paths, ordered by the path's estimated log likelihood. It is initialised with a null path, and the decoder loops 
over the following operations until a complete path is found:

1. Pop the highest scoring partial sequence off the stack.

2. For each potential next word:

(a) Extended the partial hypothesis with the candidate word, and calculate its likelihood. The new hypothesised path is then placed on the stack in a position determined by its score.

Extensions to the path are usually found using a fast match procedure [47], which is a computationally inexpensive method of narrowing down the potential next word candidates.

Stack decoding is admissible if $g^{*}(p)$ provides an upper bound of the true value of the remaining $\log$ likelihood $g(p)$. The solution found by the algorithm is optimal because the true likelihood of a path will always be less than the estimated likelihood. Therefore, any paths remaining after a solution has been found are guaranteed to have lower likelihood than that of the solution.

\subsubsection{Multi-pass decoding}

While the use of both the acoustic and language models in a single search pass is desirable, it can also present some problems. In the case of Viterbi decoding, this approach is only practical for $n$-grams of short length. Using larger $n$-gram models significantly increases the search space, and it rapidly becomes infeasible to calculate. It is also only optimal for bigrams. $N$-grams with $n>2$ violate the dynamic programming invariant assumed by the algorithm. Finally, it is not obvious how more sophisticated language models can be applied in this framework.

Although the stack decoder is capable of addressing these issues, the complexity is then shifted to finding an appropriate heuristic. Stack decoding is only guaranteed to find the optimal solution if the evaluation function is admissible. Furthermore, if the function does not provide a sufficiently tight bound, the decoder becomes very inefficient, degenerating to a breadth first search in the worst case.

The complexities involved in incorporating more advanced knowledge sources into the decoding process has given rise to multiple-pass strategies in which more sophisticated models are used to re-evaluate a set of hypotheses generated by an HMM/bigram decoder. These hypotheses are then successively re-evaluated using more sophisticated models, in a process referred to as rescoring (Figure 2.5). 


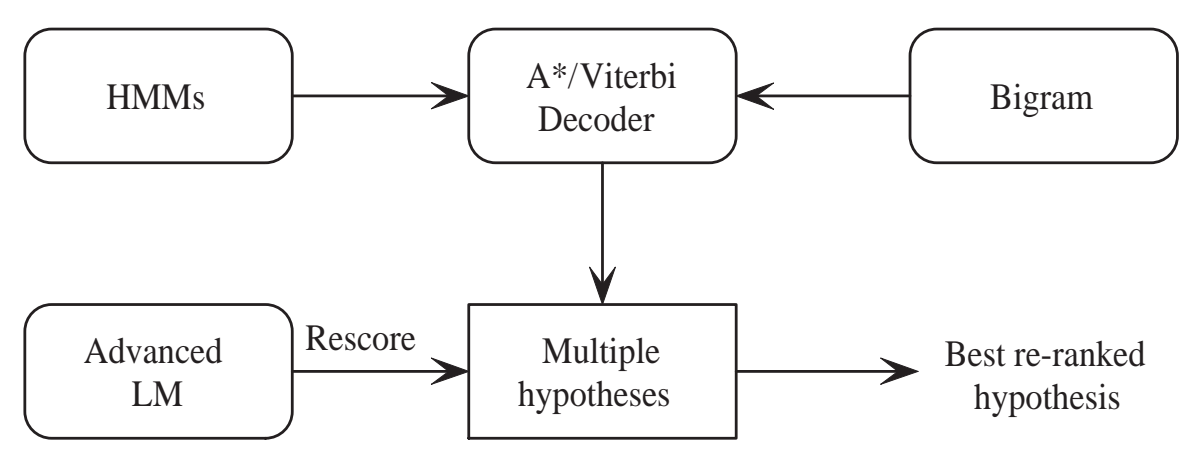

FiguRE 2.5: Generation of hypotheses for multi-pass decoding.

This approach has the advantage of greatly reduced computational complexity and improved scalability, as the more expensive knowledge sources are only applied to smaller search spaces. Separating the decoding process can also allow each component to be independently developed and optimised. Of course, the trade-off for this reduced complexity is the inability to bring all relevant knowledge to bear in the initial stage of decoding, which has the potential to propagate errors through to subsequent passes.

\section{N-best lists}

One possible interface between the different decoding passes is a ranked list of the best $N$ word sequences. This $N$-best list can be generated by a Viterbi decoder with some modifications [48]. The decoder is required to rank its output based on scores of word sequences, so the words associated with state sequences are also stored. Multiple paths are retained at each time frame, corresponding to different word histories, and if more than one path maps to the same word sequence, then their scores are summed. Up to $N$ paths are retained for the backtrace step of the algorithm, and an additional beam width can be introduced to prune paths based on word sequence probabilities.

The resulting $N$-best list contains the best $N$ hypotheses, each of which can be augmented with acoustic, language model, and other scores. A more advanced language model can then be used to calculate new language model scores, and the set of hypotheses re-ranked accordingly. This allows the language model component of the scores to be updated, while retaining the acoustic information calculated in the original pass. An example of an $N$-best list is shown in Figure 2.6. The $N$-best paradigm is useful because it decreases the search space significantly. Hence, subsequent rescoring, even with computationally expensive models can be performed 


\begin{tabular}{|lc|}
\hline 1. The latest limit index ... & -16782.5 \\
2. The latest of limit index ... & -16783.8 \\
3. The latest lemons index ... & -16788.8 \\
4. The latest limit in tax ... & -16789.5 \\
$\ldots$ & $\ldots$ \\
100. The latest of lemons index ... & -16808.9
\end{tabular}

Figure 2.6: An $N$-best list. Each sentence is annotated with a log probability score. Probabilities and time boundaries may also be specified for individual words.

quickly. Another benefit is that providing a set of complete hypotheses allows rescoring with long-distance models to be performed easily, without the need for complex decoding procedures.

\section{Word lattices}

The main drawback of $N$-best lists is that they only contain a small number of hypotheses. Reducing the search space so dramatically may result in the best hypothesis being eliminated from the list of likely candidates, thus restricting the potential improvements made by incorporating more advanced knowledge sources. Increasing $N$ allows for a larger set of hypotheses, but rescoring very large $N$-best lists is inefficient. This is particularly evident when dealing with long utterances, where competing hypotheses will often share many similar fragments. An alternative, compact representation of multiple hypotheses is provided by the word lattice. A word lattice is a directed, acyclic graph in which nodes represent words occurring at specific times, and arcs indicate allowable transitions between words. A hypothesis is then a path through this graph. The finer granularity of this representation allows lattices to provide much greater coverage than $N$-best lists without an equivalent increase in storage requirements.

A lattice can also be simplified into a word graph by merging paths which share the same word sequence. Timing and acoustic information are lost in this representation, so it is useful for rescoring with models employing linguistic knowledge only. An example of a word graph is shown in Figure 2.7.

Word lattices can be generated by a Viterbi decoder which retains information about which words end at each node during the search. The best path is extended as in standard decoding, and the additional information is used in the backtrace step to generate a lattice of hypotheses [49]. This process can be performed with minimal impact on the complexity of the decoding step. 


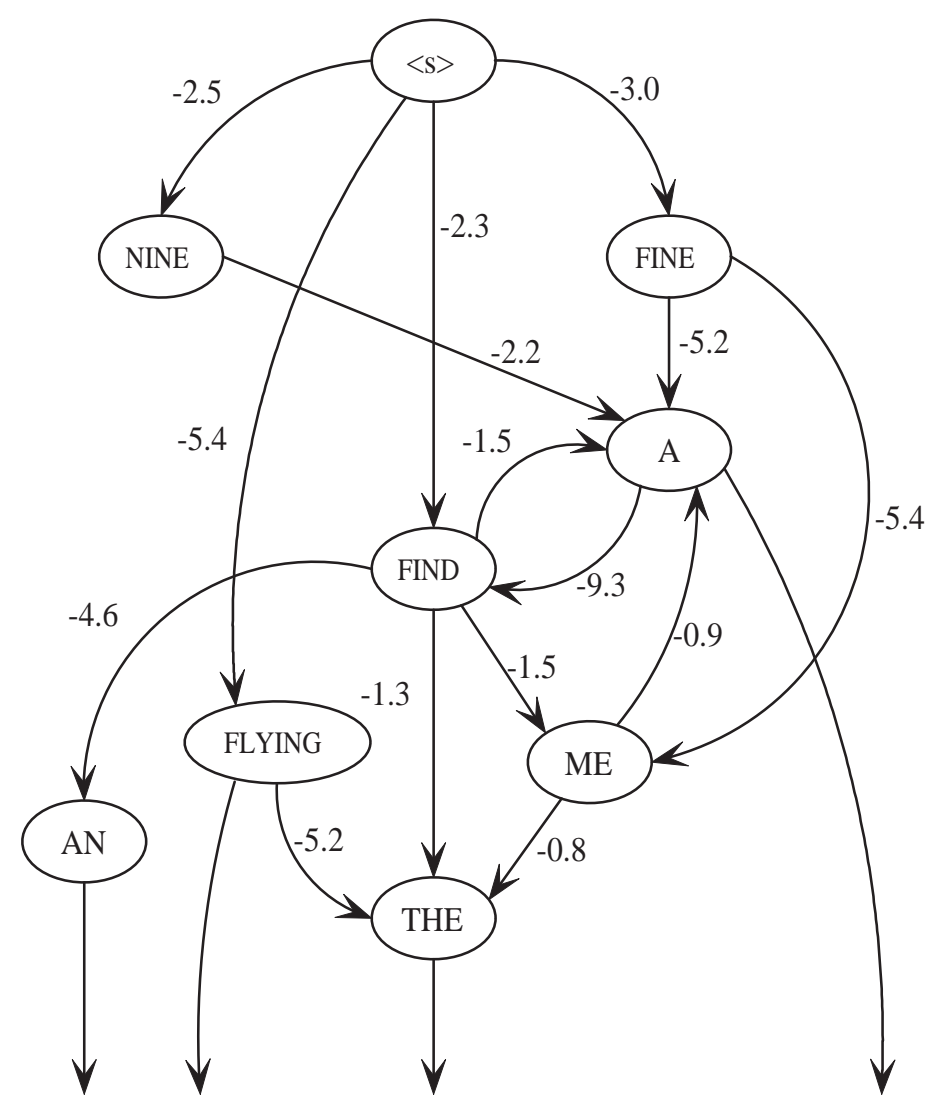

Figure 2.7: A fragment of a word hypothesis graph. Links are annotated with acoustic model (not shown) and language model log probabilities.

\subsection{Evaluation}

\subsubsection{ASR evaluation}

The performance of a speech recogniser can be measured by comparing the extent to which its output differs from a correct, reference transcription. A minimum edit distance algorithm [50] is first used to align the hypothesised transcription to the reference. Errors can then be categorised into three groups. Substitution (S) errors are the result of a word being incorrectly identified. Insertion (I) and deletion (D) errors occur when the output transcription either contains extraneous words or omits a correct word. An example of these types of errors is shown below:

reference: The trial is expected to last three to five weeks

hypothesis: The trial is expect is to last three five weeks error:

S I D


Given these counts, the Word Error Rate (WER) can be calculated as:

$$
\mathrm{WER}=\frac{D+S+I}{N} \times 100 \%
$$

where $N$ is the number of words in the reference transcription. Recogniser performance may also be reported in terms of word accuracy, which is simply 100-WER.

\subsubsection{Statistical significance}

When comparing the performance of two speech recognition algorithms, it is often useful to know if any improvements in accuracies are statistically significant. Particularly when dealing with small gains, it is important to determine whether or not the observable differences can be attributed to chance. Only when this possibility is sufficiently low may the conclusion be drawn that one algorithm is superior to the other.

One test which is appropriate for analysing results generated by continuous speech recognition systems is the Matched Pairs Sentence-Segment Word Error (MAPSSWE) Test [51, 52]. This is a parametric test which compares the number of errors made by two systems on the same data over a number of segments. These segments are required to satisfy the criterion that the errors in a given segment are statistically independent of the errors in any other segment. In the MAPSSWE test, this independence is approximated by selecting segments such that they are bounded on both sides by either two consecutive correctly recognised words, or a sentence boundary token.

If $Z_{i}$ is the difference in the number of observed errors for segment $i$ between the two systems under consideration, the estimate of the mean error in a segment is:

$$
\hat{\mu}_{z}=\sum_{i=1}^{n} \frac{Z_{i}}{n}
$$

for a sample of $n$ segments. The variance of the $Z_{i}$ 's is:

$$
\sigma_{z}^{2}=\frac{1}{n-1} \sum_{i=1}^{n}\left(Z_{i}-\mu_{z}\right)^{2}
$$

Normalising $\mu_{z}$ by its estimated standard deviation gives:

$$
W=\frac{\hat{\mu}_{z}}{\sigma_{z} \sqrt{n}}
$$


which, for large $n$, has an approximate normal distribution with unit variance [51]. The null hypothesis $\mathbf{H}_{\mathbf{0}}: \hat{\mu}_{z}=0$ postulates that $W$ has zero mean. It can be rejected if:

$$
2 \times p(Z \geq|w|) \leq \alpha
$$

where $Z$ is the standard normal distribution and $w$ is the realised value of $W$. Commonly reported values of $\alpha$ are $0.05,0.01$ and 0.001 .

\subsubsection{Perplexity}

If a language model is used for ASR, then the performance of the language model can also be evaluated in terms of WER so long as the other components remain fixed. However, it is often beneficial to be able to gauge the quality of a language model without the need for a recogniser, and this is necessary if the intended application of the language model is not speech recognition. One such measure is perplexity, a metric commonly used to compare language model performance.

Entropy, in the information theoretic sense, provides a measure of the average uncertainty associated with a random variable. The entropy of a discrete random variable $W$ is defined as:

$$
H(W)=-\sum_{w \in W} p(w) \log _{2} p(w)
$$

This can be interpreted as a lower bound on the number of bits required to encode the outcomes of $W$ given its distribution. For a Markov chain data source generating a sequence of such random variables, $w_{1}^{m}$, the per-symbol entropy, or entropy rate, $H$ of the source is given by:

$$
H=-\lim _{m \rightarrow \text { inf }} \frac{1}{m} \sum_{w_{1}^{m}} p\left(w_{1}^{m}\right) \log _{2} p\left(w_{1}^{m}\right)
$$

If the generation of words in a natural language is considered to be a Markov chain, then some additional assumptions can be made. Firstly, two occurrences of the same word may be separated by any number of words. Secondly, with enough text, it is possible to observe any word from any given starting point. These properties indicate that natural languages are both aperiodic and irreducible, and therefore ergodic. The entropy rate of an ergodic Markov chain reduces to:

$$
H=-\lim _{m \rightarrow \inf } \frac{1}{m} \log _{2} p\left(w_{1}^{m}\right)
$$


With a sufficiently large $m$, this can then be approximated by:

$$
\hat{H} \approx-\frac{1}{m} \log _{2} p\left(w_{1}^{m}\right)
$$

which provides an estimate of the entropy rate of the language.

A language model can only approximate of the true distribution of the language, so cross entropy is used to compare the two distributions. The per-word cross entropy of the true distribution $p(w)$ given the model's estimate, $\hat{p}(w)$ is defined as:

$$
H(p, \hat{p})=-\lim _{m \rightarrow \inf } \frac{1}{m} \log _{2} \hat{p}\left(w_{1}^{m}\right)
$$

By applying a similar argument to that used to obtain Equation $(2.15)$, this can be reduced to:

$$
H(p, \hat{p})=-\frac{1}{m} \log _{2} \hat{p}\left(w_{1}^{m}\right)
$$

This gives the per-word cross entropy in terms of the model $\hat{p}$ and an empirical sample, so it then becomes possible to use this to measure a model's quality. A property of the cross entropy is that:

$$
H(p) \leq H(p, \hat{p})
$$

As the model estimate $\hat{p}$ becomes closer to the true distribution, the cross entropy approaches the entropy of the language. Therefore, the better the model, the lower the cross entropy will be. Although it is possible to evaluate language models using cross entropy, it is more common to use the closely related measure of perplexity:

$$
P P=2^{H(p, \hat{p})}
$$

Perplexity has an intuitive interpretation as the average number of equiprobable choices the model encounters when presented with a sample of the language. 


\section{Chapter 3}

\section{Prosody}

A spoken language contains information beyond that which is available in its textual representation. A speaker can control properties of an utterance in order to convey additional information on semantics, syntax and pragmatics. Paralinguistic information, such as attitude, emotional state, age and gender, can also be communicated through speech. These types of information are largely signalled through variations in the prosodic features of pitch, loudness and length. While the precise relationship between prosodic features and their linguistic and paralinguistic functions has proven difficult to characterise, it is clear that prosodic information plays an important role in human comprehension of spoken language.

This chapter will present an introduction to prosody and examine its relevance to ASR. The first section will describe the perceptual and acoustic correlates of prosody, and note commonly used techniques for automatically extracting this information from a speech signal. In the second section, an overview of the functions of prosody will be provided, focusing in particular on the role prosody plays in human-tohuman communication. The high degree of variability in the use of prosody between languages and speakers will also be highlighted. The third section will describe some approaches to modelling prosody. It will cover Pierrehumbert's model of intonation and an annotation scheme based on the model. The applicability of the Tones and Break Indices annotation framework to automatic speech processing systems will be examined, and some alternatives discussed. The fourth section will investigate the ways in which prosody may be of use to ASR, and will include a review of previous work in this area. This chapter will conclude with a description of how prosodic knowledge can be incorporated into a language model for ASR, and a discussion of the advantages and disadvantages of doing so. 


\subsection{Prosodic Features}

The prosody of an utterance is often represented as a sequence of prosodic units at the linguistic level. These units are tone, intonation and stress, and can be expressed as variations in the perceptual attributes of pitch, loudness and length.

\subsubsection{Pitch}

Pitch is the perceived fundamental frequency of a sound. The excitation of a voiced speech sound is caused by vibration of the vocal folds. The glottis periodically opens and closes, and the reciprocal of this period is the fundamental frequency (F0). Speech sounds can also be produced when the vocal folds do not vibrate. These are unvoiced sounds, and are not associated with any F0. The perceived F0 may differ from the actual F0, as auditory pitch perception can be influenced by harmonic structure and amplitude [53].

Automatic detection of fundamental frequency contours is complicated by a number of factors. Speech is not truly periodic, and F0 can change with each glottal period. Voicing onset and offset may be irregular, and voiced intervals may be very short, leading to errors in voicing detection. Also, high energies in the harmonics can lead to frequency doublling or halving errors. A widely used pitch detection algorithm [54] is based on the normalised cross correlation function (NCCF). For a sampled signal $s(n), 0 \leq n \leq N-1$, the NCCF for a lag of $k$ is given by:

$$
\operatorname{NCCF}(k)=\frac{1}{\sqrt{e_{0} e_{k}}} \sum_{n=0}^{N-k} s(n) s(n+k)
$$

where

$$
e_{k}=\sum_{n=k}^{k+N-K} s^{2}(n)
$$

for $0 \leq k \leq K-1$, where $K$ is the maximum number of lags computed. If the signal $s(n)$ is periodic, then the NCCF is also periodic and exhibits peaks at the periodicity of the signal. The locations of the two largest peaks can then be used to determine the pitch period [55].

\subsubsection{Loudness}

Loudness is the perceived amplitude of a sound, and is approximately proportional to the logarithm of the intensity. Loudness is also affected by the signal's frequency. The human ear is sensitive to frequencies between $3000-4000 \mathrm{~Hz}$, so a signal 
lying in this range will be perceived to be louder than a signal with equal intensity outside this frequency range. Similarly, sounds with a frequency below $300 \mathrm{~Hz}$ will be perceived as being softer due to the ear's reduced sensitivity in this region.

Loudness metrics corresponding closely to perception have been proposed [56]. It is more common, though, to use short-time root mean square (RMS) amplitude as an estimate of loudness. For a frame $j$ containing $N$ samples, $a_{j 1 \ldots j N}$, the RMS amplitude is calculated as:

$$
R M S_{j}=\sqrt{\frac{1}{N} \sum_{i=1}^{N} a_{j i}}
$$

where $a_{j i}$ is the squared magnitude of the $i^{\text {th }}$ sample in frame $j$ :

$$
a_{j i}=|s(j N+i)|^{2}
$$

\subsubsection{Length}

Length encompasses a number of duration-based features. These include segment, syllable and pause durations, as well as speaking rate and rhythm.

Speaking rate can be calculated as the reciprocal of the average of the phone durations within a certain time interval [57]. In this calculation, the phone durations must be normalised in order to preclude the effects of phone intrinsic values [58]. Also, speaking rate is not linearly related to segmental durations. Durations are highly context dependent [59], and temporally compressing the segments of an utterance by a fixed value results in unnatural sounding speech. On average, consonants have higher duration variability than do vowels, particularly if the vowels are stressed.

Rhythm refers to the temporal organisation of strong syllables (containing full vowels) and weak syllables (containing reduced vowels) [60], and is largely perceptual. There are many factors which affect durations of speech sounds, and it can be difficult to isolate those sources of variability which determine the perception of rhythmic structure [61]. Examples of such rhythmic phenomena are compensatory shortening, in which stressed syllable durations decrease as the number of succeeding syllables increases, and final lengthening, where the durations of the final unstressed syllable in an utterance are significantly longer than those of other unstressed syllables. 


\subsubsection{Stress}

Stress is a combination of pitch, loudness and length effects. Some syllables are pronounced with greater prominence than other syllables in their context. These stressed syllables are characterised by higher fundamental frequency and amplitude, and longer durations [62], although some experiments have found the influence of F0 to be minimal [63]. Although stress always occurs on individual syllables, there can be multiple levels of stress. These can be associated with words or syntactic groups of words, termed word stress and phrasal stress, respectively.

\subsubsection{Tone}

Tones, or tone levels, refer to distinct functional patterns of pitch, typically at the syllabic level. The precise number of tones varies depending on the language. Mandarin Chinese, for example, has four tones (high, rising, low and falling), while Thai has an additional mid tone. In English, a distinction is made between only two tones, high and low.

\subsubsection{Intonation}

Patterns of controlled pitch variation over the longer prosodic domains, such as the phrase or sentence, are referred to as intonation. Pitch is affected by physiological factors such as age and gender. These influences are outside the control of the speaker and do not convey linguistic information. Therefore, it is the relative pitch movements that are important to intonation analysis, rather than absolute pitch values. A human listener can consider an intonation contour to be a successful imitation of another contour despite noticeable perceptual differences. Such perceptually equivalent [64] intonation patterns are assumed to have the same phonological structure and therefore convey the same linguistic information.

\subsection{Functions of Prosody}

Prosody conveys a diverse range of information. At the lexical level, it affects the acoustic realisations of phonemes and words. Prosody also influences higher level linguistic structures such as syntax and semantics, and provides paralinguistic information such as emotion. Some of the major linguistic functional roles of prosody include:

- Distinguishing between phonetically similar words (lexical distinction). 
- Aiding in structural processing.

- Providing discourse information.

Examples of how prosody performs these roles in human communication, and which features are involved in these processes, are described below.

\subsubsection{Roles in human communication}

The importance of prosody in human speech comprehension has been demonstrated in numerous studies. Experiments with nonsense syllables are often used in order to minimise the effects of subjects' prior knowledge. Under auditory presentation of nonsense syllables, prosody has been found to facilitate the ability to recall these sequences [65]. Confusions in recognition of nonsense bisyllables are more likely to occur between those bearing the same stress pattern [66]. Given a pair of these bisyllables, such as [bisev, jubim], listeners misrecognised them more frequently when presented with [bibim, jusev] as opposed to [bisev, jubim]. Similar behaviour can also be observed in the recognition of incorrectly stressed English words spoken by non-native speakers [67]. However, this incorrect stressing also results in segmental differences, and the possibility that the recognition errors are caused by segmental information alone cannot be ruled out [1].

Shadowing tasks, which require a listener to repeat words as they are heard, give an indication of the latency involved in human word recognition. Subjects' performances on shadowing of grammatical strings were shown to be aided by the presence of prosody [68]. In an experiment by Darwin [69], listeners were presented with a different speech stream in each ear, and asked to shadow the speech from the left or right ear only. At some point during the speech, the sources were switched. An example of this (taken from [70]) is shown below.

\begin{tabular}{l|l} 
L: an impression of grace and & is idiotic idea of almost there is cabbage \\
R: it was jumping in the tree & charm and a distinct suggestion of watchfulness
\end{tabular}

Listeners in this experiment demonstrated a tendency to reproduce speech from the incorrect stream if there was a prosodic discontinuity at the point when the streams were swapped. Significantly fewer errors were observed if the discontinuities were only semantic in nature, indicating that the retention of prosodic continuity is useful in speech perception. Similarly, disruptions to prosodic structure can result in degradation in word target recognition times [71] and semantic comprehension [72].

In experiments performed using spectrally rotated speech, humans were found 
to perform well on word recognition tests [73]. Cues to phonetic identity are predominantly spectral, so corrupting the spectra destroys phonetic information while retaining prosodic phenomena such as pitch, loudness and duration. Sentence recognition experiments showed subjects were also able to correctly recognise function words as well as retrieve syntactic information on speech corrupted in this way. The results of these experiments indicated listeners were able use whichever acoustic cues were available, as well as weight them appropriately according to their relative information content.

It has also been suggested that prosody interacts differently with closed-class and open-class words. A closed class is a class of words to which new terms cannot normally be added, such as prepositions, determiners and pronouns. The set of closed class words is relatively small, but these words occur frequently in natural languages. They are also acoustically low in salience, making them difficult to recognise in isolation. In contrast, open classes such as nouns and verbs readily admit new members. Open-class words tend to contain more information content, while closed-class words are generally associated with structural roles. Recognition of closed-class words has been found to be highly dependent on prosodic context, while recognition of open-class words exhibits less reliance [74].

Given a stream of continuous speech, successful word recognition requires the identification of word boundaries. Speech contains no direct equivalent of the punctuation and whitespace present in text, yet processing of speech requires a decomposition of the utterance into smaller units. In order to perform this segmentation, listeners must rely on acoustic cues which are not completely reliable [6]. Duration is one potential indicator of boundary location. There are durational differences between a syllable occurring as a monosyllabic word and the same syllable at the start of a polysyllabic word, which may be exploited to infer word boundaries. Rhythmic properties may also cue boundary locations. English listeners favour segmentation before strong syllables, and can more easily identify words with strong-weak syllabic patterns. For example, listeners recognised a given word, such as mint, more quickly if it preceded a monosyllabic suffix containing a weak vowel (esh) than if it preceded one with a full vowel (ayve) [75].

Prosody is also relevant in the segmentation of utterances into units above the word level. Like syntactic structure, phonological structure is typically regarded as being hierarchical, and the organisation of utterances into intermediate and full intonational phrases is cued by prosodic information. While prosodic structure is not isomorphic with syntactic structure, boundaries of intonational phrases often correspond to syntactic boundaries [76], and major syntactic boundaries can be ac- 
curately determined using only prosodic information [8]. Consequently, prosodic boundaries can be used to disambiguate some utterances which are otherwise syntactically ambiguous [6]. Acoustic cues of these prosodic boundaries include preboundary lengthening of segments, and pitch rise or fall-rise patterns. In English, low and medium strength boundaries are signalled primarily by durational features, while major boundaries are closely related to both pitch and duration correlates [77].

The role of prosody in grouping and segmentation of speech units has also been demonstrated in experiments using disrupted speech, such as an utterance containing a superimposed click. Listeners, after being presented with the disrupted utterance, were asked to recall the position in which the click occurred. If prosodic boundaries were consistent with syntactic boundaries, then the perception of disruptions tended to move toward these boundaries. When prosodic and syntactic structure conflicted, though, this effect was not observed [78], suggesting that prosody is used by listeners to assist with syntactic parsing.

Another possible contribution of prosody in structural processing is local ambiguity resolution. Syntactic ambiguities of an incomplete utterance may be resolved in an early processing step through the use of prosodic information. Consider the two endings to the following sentence:

$$
\text { The city council argued the mayor's position } \mid \begin{aligned}
& \text { forcefully. } \\
& \text { was incorrect. }
\end{aligned}
$$

With the first ending, the mayor's position is the direct object of argued, while with the second ending, it is the subject of a sentence complement. In experiments in which English listeners were presented with only the syntactically ambiguous fragment of such sentences, the listeners were able to determine which of the variants the fragment belonged to [79]. Similar experiments with sentence onsets found listeners were also able to decide whether the sentence contained no more words, three more words, or six or more remaining words [5]. Pitch and duration features of the last presented word were the primary cues employed by the subjects in these tasks [80].

At the higher levels of the spoken communication process, prosody plays a role in a number of discourse functions. As with lexical and syntactic functions, though, not all discourse functions are realised prosodically. Functions which are at least partly cued by prosody include conveying speaker attitudes, turn-taking, feedback and speech act signalling. Prosody is also used to give prominence to the salient information in a discourse [4]. The accentuation of a particular word is dependent on the importance of that word in the sentence, as well as its context, and these 
words are more easily processed by listeners. While the increased acoustic clarity of stressed syllables associated with accented words improves speed and efficiency of comprehension, it is not the sole reason for this effect. In an experiment using acoustically identical copies of an accented word in two different prosodic contexts, faster reaction times in recognition of the accented word were observed for the case when prosody was consistent with an accent in the target location [81]. While manipulation of either pitch or duration alone did not hamper this effect, consistency between the various prosodic factors appeared to be important [82].

The accent structure of an utterance is related to its semantics, and in particular, its focus. Whether an item represents given or new information in a discourse affects a speaker's decisions about accentuation and deaccentuation. Grammatical function and word position are also factors contributing to this choice [83]. The relationship between accentuation and new or given information is exploited by listeners in speech comprehension, particularly when recognition of segmental identity is obscured [84]. In these situations, accented words are more likely to be interpreted by listeners as new information.

Prosody can also provides cues to the identification of topics in discourse. Topics are often started high in the speaker's pitch range and show a rise in amplitude, while the end is associated with a compressed pitch range and amplitude fall [85]. Corpus-based studies using professionally read speech have found statistically significant correlations between discourse segmentation and prosodic features including pitch range, amplitude and timing [86]. These correlations were evident with both discourse elements obtained from the text only, as well as those obtained from a combination of text and speech.

Prosodic continuity is an important factor in speech processing. Conversely, disruptions to this continuity can cause difficulties in comprehension, even if the speech is otherwise fluent. Prosody may then act as an indicator of disfluencies such as hesitations and speech repairs. Disfluencies are characterised by an error, also known as a reparandum, followed by an editing phase, and a subsequent correction to the reparandum. They may occur for various reasons, including problems with lexical retrieval or speech planning. Hesitations are associated primarily with pausing and syllable lengthening [87]. While textual properties, such as the presence of word fragments [88], are good indicators of speech repairs, acoustic and prosodic cues to these events also exist. Silent pauses, especially, tend to be good indicators of interruptions, and there are durational differences between these types of pauses when compared to the pauses present in fluent speech [89]. Glottalisation at the end of the reparandum is also commonly observed [89], particularly in the case of vowel-final 


\begin{tabular}{lll}
\hline Acoustic Correlates & Perceptual Correlates & Functions \\
\hline \hline F0 level, f0 contour & $\begin{array}{l}\text { Pitch level, pitch contour, } \\
\text { tone, intonation, stress }\end{array}$ & $\begin{array}{l}\text { Lexical distinction, prominence, } \\
\text { focus, grouping into intonational } \\
\text { phrases, discourse segmentation }\end{array}$ \\
\hline Amplitude & Loudness, stress & Prominence, focus, segmentation \\
\hline $\begin{array}{l}\text { Segment duration, } \\
\begin{array}{l}\text { syllable duration, } \\
\text { silence }\end{array}\end{array}$ & $\begin{array}{l}\text { Rhythm, pause, stress } \\
\text { stress }\end{array}$ & $\begin{array}{l}\text { Prominence, focus, grouping, } \\
\text { segmentation, disfluencies }\end{array}$ \\
\hline
\end{tabular}

TABLE 3.1: The relationships between the acoustic and perceptual realisations of prosody, and their major linguistic functions.

fragments.

The relationships between the various aspects of prosody are complex and difficult to isolate. Each of the prosodic features of F0, amplitude and duration, provide cues to a number of linguistic functions. Similarly, the prosodically-dependent functions are influenced simultaneously by multiple features. An attempt to summarise the interaction of the acoustic and perceptual correlates of prosody, as well as their major prosodic functions is shown in Table 3.1.

\subsubsection{Cross-linguistic differences}

Prosody is present in all spoken languages; however, its functions are largely language dependent. While there is some evidence to support a universal mechanism for communicating certain types of emotion [90], the linguistic aspects of prosody vary greatly between languages.

One notable distinction is found in the use of tones. In some languages, called tonal languages, tones perform a lexical role. These languages contain words which consist of the same phonemic sequences but are distinguished by different tones. The tones therefore form part of a word's identity, and are necessary to determine its meaning. There are two types of tonal languages. In tone languages, each syllable is associated with its own tone. Most South-East Asian and African languages, such as Mandarin, Thai and Yoruba are tone languages. In pitch accent languages, such as Japanese and Swedish, words, rather than syllables, are associated with distinct tonal melodies. Because these pitch accents perform the same role as tones in tone languages, they therefore are not used to signal focus and information structure.

In contrast to tone languages, stress languages do not use tones directly in the lexicon. Some of these languages use stress, of which pitch is a component, to perform a similar role. In English, the noun and verb forms of certain words are 
distinguished by their stress pattern (e.g. SUBject vs subJECT). In other stress languages, stress placement is predictable, always occurring on the same syllable. In Finnish, the first syllable of a word is stressed, and in Polish, stress falls on the penultimate syllable. Stress is also used to indicate focus on words. For example, in the sentence The meeting is on MONDAY, not TUESDAY, stress effects are more pronounced on the words Monday and Tuesday than on meeting, even though the first syllable of meeting is also stressed.

Prosodic indicators of word boundaries are also language specific. Segmentation is performed using rhythmic cues; however, the nature of these cues differ depending on the language. For instance, the rhythmic structure of English is described in terms of stress (it is a stress-timed language), while French is syllable-timed and Japanese is based on a constituent of the syllable called a mora (usually a consonant-vowel pair). In addition, the use of segmentation cues is highly dependent on the listener. While the French language is optimally segmented based on syllable structure, Japanese listeners of French speech do not perform this [91]. Similarly, French listeners of Japanese speech showed no evidence of using mora-based segmentation [92].

Given the amount of variation in these fundamental aspects of prosody, it is unsurprising that the higher level linguistic functions of prosody also exhibit many cross-linguistic differences. Furthermore, different dialects of the same language can have considerable differences in how prosody is used to signal syntactic and semantic information. The production and interpretation of prosodic cues can even vary between different speakers [93]. This study will focus on one particular speaking style only. The database used here consists of professionally read American English speech, and will be described in more detail in Chapter 5.

\subsection{Modelling Prosody}

Differing motivations for studying prosody have resulted in a wide range of representations of prosodic information. Linguistic studies have focused on the phonological aspect of prosody, giving rise to models of phonological constituents. At the other end of the scale, the problem has been approached with regard to the acoustic and phonetic properties, which are often more directly applicable to the automatic processing of prosodic information. In general, though, a model of prosody should be capable of describing the full range of prosodic phenomena in terms of both phonological units and their acoustic realisations. 


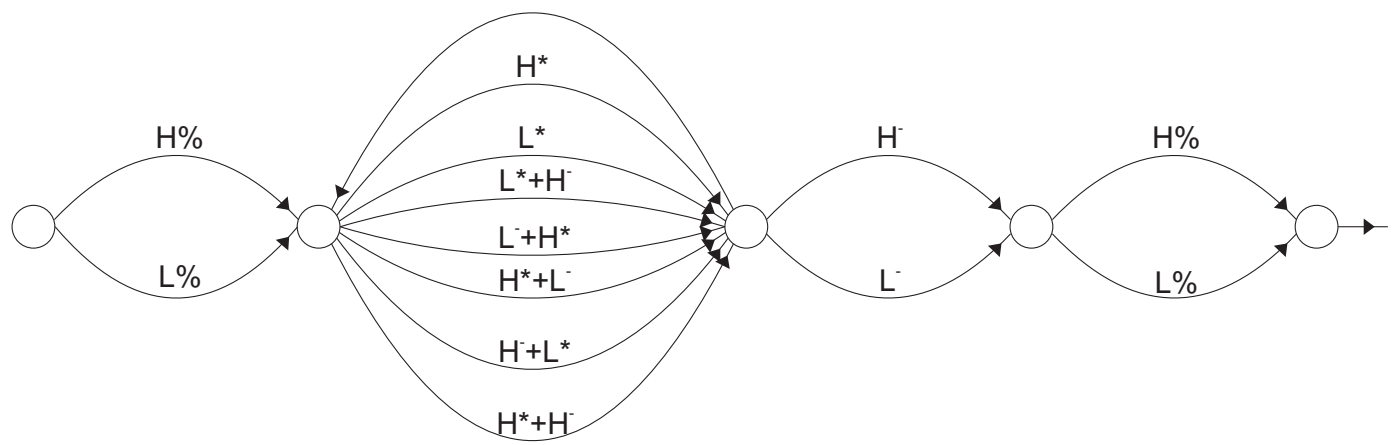

FiguRE 3.1: Finite-state grammar of allowable tone sequences.

\subsubsection{Pierrehumbert's model}

Much attention in prosodic studies has been devoted to analysis of the role of intonation. An influential work in intonational phonology has been that of Pierrehumbert [94]. Her proposed model of English intonation describes an intonation contour as a linear sequence of high $(\mathrm{H})$ and low (L) tones, where high and low are defined relative to the local pitch range. Two sets of rules are used to map these tones to F0 values. The tones themselves define context-dependent pitch targets. Global pitch declination can be encoded in this approach by lowering target pitch values at specific accents, a process referred to as downstep. The second set of rules are used to specify the interpolation between pairs of pitch targets based on a local region of the tonal sequence.

A distinction between Pierrehumbert's model and that of previous work [95] is the use of two tones rather than four, which Pierrehumbert argued contained too much ambiguity given the variability of pitch range [96]. The tones fall into three categories based on the domain on which they operate. Pitch accents, which may be either single tone $\left(\mathrm{H}^{*}, \mathrm{~L}^{*}\right)$ or bitonal $\left(\mathrm{H}^{*}+\mathrm{L}, \mathrm{H}+\mathrm{L}^{*}, \mathrm{~L}^{*}+\mathrm{H}, \mathrm{L}+\mathrm{H}^{*}, \mathrm{H}^{*}+\mathrm{H}\right)$, describe the tonal patterns of stressed syllables, where the ${ }^{*}$ indicates the placement of the accent. The onset and offset pitch of an intonational phrase are controlled by boundary tones (H\%, L\%) aligned with the edges of the phrase, and the pitch movement in the region between the last pitch accent and final boundary tone is specified by the phrase accent $(\mathrm{H}, \mathrm{L})$.

The sequential organisation of tones is defined by the finite-state grammar shown in Figure 3.1. For each intonational phrase, the grammar specifies legal tone sequences as comprising of an initial boundary tone, one or more pitch accents, and followed by a mandatory phrase accent and final boundary tone. Any tone sequence conforming to this structure is considered valid by the grammar, and all such se- 
quences are also well-formed in English according to Pierrehumbert. The model uses a metrical grid [95], which specifies the patterns of stressed and unstressed syllables, as well as the relative strengths of stressed syllables. A set of rules are also defined for aligning this metrical representation of the text with the tone sequence.

The Pierrehumbert model provides both a description of prosody at a phonological level and a mapping from phonology to F0 contours. However, it does not specify a mapping in the opposite direction. In fact, Pierrehumbert claims that such a mapping does not exist. This point has been criticised by Taylor [97], who believed the problem to lie with the interpolation rules which he considered too unprincipled. The lack of a F0 to phonology mapping poses problems for automatic processing, as F0 contours can often be the only available source of prosodic information. It is possible, though, to obtain a phonological representation from acoustic data by means other than inverting the original interpolation rules. Such approaches [98, 99, 100] rely on statistical methods to perform labelling based on acoustic similarities.

\subsubsection{Tones and Break Indices}

The ideas evinced in Pierrehumbert's model have been adapted into the Tones and Break Indices (ToBI) framework [101] for labelling prosodic phenomena. The development of ToBI was motivated by the need for a standardised system for transcribing prosody, and was targeted in particular at corpora development. While originally designed for American English, ToBI systems for other languages have also been developed. The English ToBI system attempts to capture two types of prosodic events in particular: the intonation patterns, and the rhythmic structure of stressed and unstressed words. Those events which are not suited to categorical representations are left out, as are events which can be reliably predicted, such as the location of stressed syllables within a word [102].

Labels for events are organised into four parallel tiers:

- Orthographic tier

- Tone tier

- Break index tier

- Miscellaneous tier

Each tier describes a specific stream of information, with the core prosodic analysis contained in the tone and break index tiers. All tiers are also temporally aligned with both the original utterance and its F0 contour. 
The orthographic tier contains the transcribed words of the utterance in standard English orthography. These words are marked at the end of their final segments. Comments and any phenomena which cannot be transcribed onto the tone or break index tiers are placed in the miscellaneous tier. Examples of events marked on this tier include disfluencies and audible breathing.

The tone tier contains the phonological analysis of the utterance's intonation contour. It corresponds closely to Pierrehumbert's representation, although there are some differences. ToBI defines an intermediate intonational phrase in addition to the full intonational phrase. Adopted from revisions of Pierrehumbert's model [103], the intermediate intonational phrase contains one or more pitch accents followed by a phrasal tone, which is distinct from the boundary tone. Downstepping is marked explicitly in ToBI (as ! $\mathrm{H}^{*}$ ), in contrast to Pierrehumbert's approach in which downstepping was triggered by certain tone sequences. The explicit annotation of downstepping eliminates the need for the $\mathrm{H}^{*}+\mathrm{L}$ downstep trigger; $\mathrm{H}^{*}+\mathrm{L}$ has been removed from ToBI's inventory of tones.

The full set of tone labels described by ToBI are [104]:

- L-, H- phrase accents, occurring at the boundaries of intermediate or full intonational phrases.

- L\%, H\% final boundary tones, occurring at full intonational phrase boundaries.

- $\% \mathbf{H}$ high initial boundary tone, indicating that a phrase begins high in the speaker's pitch range. Mid or low pitch range phrase onsets are assumed by default.

- $\mathbf{H}^{*}$ peak accent, representing a tone target on an accented syllable which is in the upper or middle part of the speaker's pitch range for the phrase.

- $\mathbf{L}^{*}$ low accent, representing a tone target which is in the lowest part of the speaker's pitch range.

- $\mathbf{L}^{*}+\mathbf{H}$ scooped accent, representing a low tone target followed by a sharp rise to a peak in the upper part of the speaker's pitch range.

- $\mathbf{L}+\mathbf{H}^{*}$ rising peak accent, representing a high tone target immediately following a sharp rise from the lowest part of the speaker's pitch range.

- $\mathbf{H}+$ ! $\mathbf{H}^{*}$ downstep onto an accented syllable from a high pitch or preceding high pitch accent. 
Pitch accents are aligned with accented syllables, while phrasal tones are aligned to the end of intonational phrases. Full intonational phrases consist of one or more intermediate phrases, therefore boundaries of full intonational phrases are marked with both a phrase accent and a boundary tone.

The break index tier represents the other component of ToBI's prosodic analysis. Break index values indicate the perceived degree of juncture between pairs of words, and between utterance final words and the following silence. This tier provides a representation of the rhythmic characteristics of an utterance, complementing the tone tier's description of the intonation. Five levels of break indices are defined in ToBI:

- 0 boundary with clear phonetic indicators of a clitic group.

- 1 regular word boundary.

- 2 boundary marked either by a pause but no tonal indicator, or an unexpectedly weak intonational boundary.

- 3 intermediate intonational phrase boundary.

- 4 full intonational phrase boundary.

This approach of labelling word boundary strengths derives from Price et al. [77] who proposed seven break index levels. Their system additionally defined break index 5 as boundaries between groups of intonational phrases, and break index 6 as sentence boundaries. The major prosodic breaks in the original system, corresponding to break indices of 4,5 , and 6 , have been merged into the full intonational phrase boundary (break index 4) in ToBI. A sample utterance, along with its orthographic, tone and break index transcriptions, is shown in Figure 3.2 .

There is some redundancy between the tone and break index tiers. By definition, break indices of 3 and 4 occur at intermediate and full intonational phrase boundaries, respectively. Therefore, these breaks can then be determined solely from the tone tier. A problem arising from this is that the limited number of remaining labels can restrict the ability of the transcriber to mark perceptual differences in relative boundary strengths [105].

Another issue with ToBI, and transcription systems in general, is the level of consistency which can obtained by different labellers. Ideally, all labellers transcribing the same utterance should produce identical transcriptions. An explicit design goal of ToBI was to achieve an inter-transcriber agreement of at least $80 \%$ [101]. 


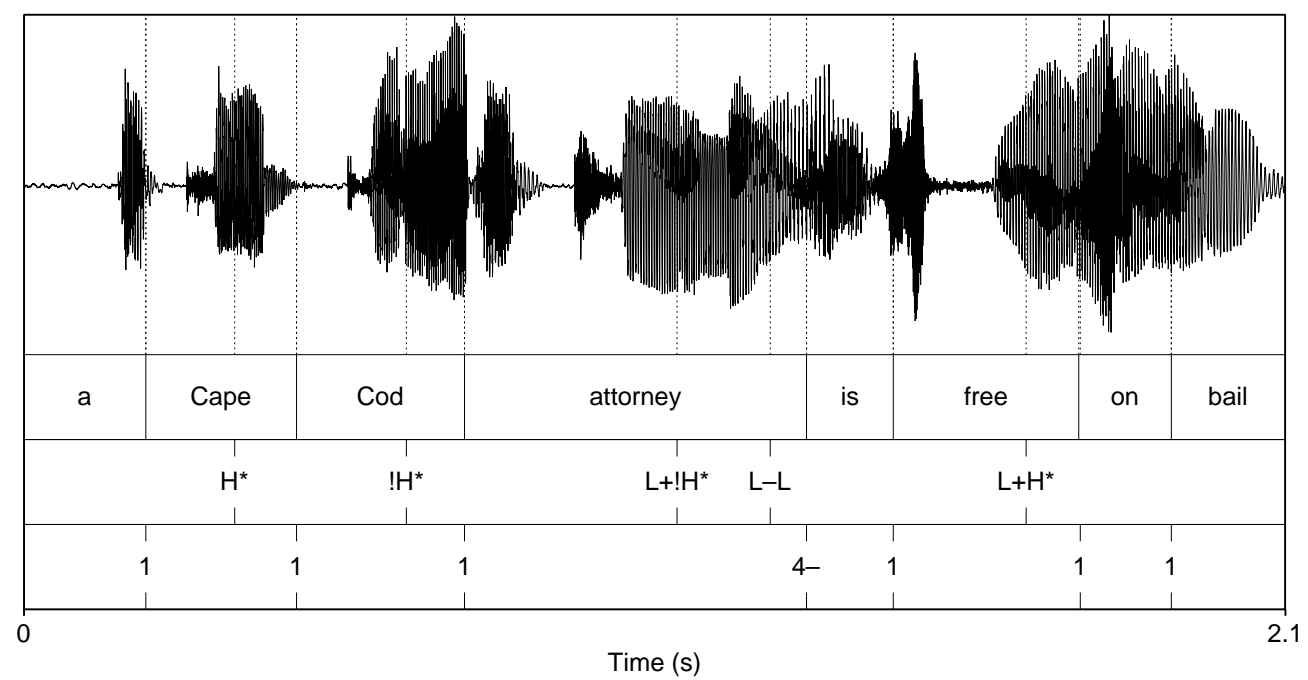

FiguRE 3.2: A ToBI transcription of an utterance.

A study of inter-transcriber consistency conducted on an English corpus [106] reported an $81 \%$ pairwise agreement on the presence versus absence of pitch accents. Similarly, agreement on the presence of phrase accents and boundary tones were $90 \%$ and $91 \%$ respectively. Agreement on break index values within a margin of \pm 1 levels was $92 \%$. The data used in this study covered a wide range of speech styles, including both read and spontaneous speech. Transcribers also had varying levels of experience, with 6 out of the 26 having had no prior experience with prosodic transcription. A separate study by Syrdal and McGory [107], in which all transcribers had the same amount of training, reported even higher consistencies.

While inter-transcriber consistency on the presence and absence of labels is high in general, agreement on specific label identity is significantly lower. In Syrdal and McGory's study [107], agreement on pitch accent type for male speakers exceeded $50 \%$ for only two types of accent: $\mathrm{H}^{*}$ and $\mathrm{L}+\mathrm{H}^{*}$. In the other categories, three of six boundary tones, and two of five break indices had an agreement above this value. All phrase accent agreements were also below 50\%. While the results for females were slightly higher (four pitch accents and one phrase accent exceeded 50\%), the overall consistencies were still low, especially considering that all labellers in this study received identical training. 


\subsubsection{Alternate modelling approaches}

ToBI is the most widespread prosodic annotation scheme for English. Its advantages include a solid foundation in phonological theory combined with clear guidelines for labelling speech data. It can describe both intonational and rhythmic phenomena, and a number of tools are available to aid and streamline the labelling process. Intertranscriber agreement is also high when using a reduced set of labels (i.e. presence versus absence), and this aspect of ToBI has been investigated more comprehensively than for other labelling approaches.

ToBI is not without its drawbacks, however, particularly when considered in the context of speech technology applications. A characteristic of most speech processing systems is the use of statistical learning methods, which require a large amount of training data. Labelling speech in accordance with the ToBI framework requires both highly trained transcribers and significant time investment. It commonly takes between 20 - 30 minutes to label 10 seconds of speech [108], so creating sufficiently large corpora is problematic. The difficulties involved in manually transcribing speech has led to the development of automatic or semi-automatic methods for assisting the annotation of utterances. Syrdal et al. [108] automatically assigned default labels based on predictions from the text, which were then manually corrected. This decreased the average labelling times without any significant introduction of labelling bias. Wightman et al. [109] used a decision tree to predict a collapsed set of ToBI labels, and reported a $69 \%$ accuracy for recognition of pitch accents and 93\% accuracy for phrase boundaries when compared to manual labels.

The possibility of accurate automatic detection and assignment of ToBI labels then raises the question of whether such a step is necessary at all. Obtaining the phonology of an utterance is rarely the final goal of a speech processing system; rather, it is used as an intermediate representation. Batliner et al. [110] note that if a mapping exists from acoustic data to phonological elements, and if there is a subsequent mapping from these constituents to a target linguistic category, then a direct acoustic to target mapping also exists. This direct modelling approach [111] brings about some practical advantages. If the acoustic to linguistic mapping can be learnt automatically, the need for time consuming manual transcriptions is removed. This allows for the use of much larger data sets, as well as eliminating the possibility of human-induced errors in the transcription process. Acoustic features can also be selected and weighted in order to optimise performance for a given task. Using an intermediate representation can potentially discard useful information or introduce quantisation errors, and this can be avoided in the direct modelling method.

The approach described by Kompe [57] represents a step away from the use of 
phonological representations in automatic speech processing. They defined a set of boundary and accent labels which are distinguished as being either syntactic, prosodic-syntactic or prosodic. Syntactic labels can be obtained from textual data only, while prosodic labels are based on perception and analysis of the F0 contour. The prosodic-syntactic labels are syntactic labels organised with respect to their expected prosodic characteristics. Automatic labelling from acoustic features was also a primary consideration in the selection of these labels. The prosodic-syntactic labels were shown to be particularly useful in training statistical classifiers.

\subsection{Prosody in ASR}

Traditionally, prosodic information has not been utilised in speech recognition systems. It is the spectral component of speech which carries the most important cues to phonetic identity, and models designed to use short-time spectral features, such as HMMs, are not well suited to modelling longer duration phenomena. Prosodic features such as F0 are also highly speaker-dependent, which is an undesirable property in most ASR applications.

Some of the earliest suggestions for using prosody in automatic speech processing were presented by Lea [112], who proposed the use of prosodic cues to detect sentence and phrase boundaries, as well as some syntactic properties of words. Waibel [7] was able to reduce the average rank of the correct word recogniser hypothesis by rescoring them with a variety of prosodic knowledge sources. Pitch, duration, stress, and especially intensity, were found to contribute to the gains. In addition, using a combination of prosodic and phonetic features resulted in further improvements in performance.

Efforts have also been made to incorporate prosodic information into the HMM framework. Distinct phone models have been used which are dependent on prosodic factors, such as lexical stress [113] and phrasing [114]. Prosody can affect the spectral properties of phonemes. For example, phrase initial vowels exhibit more coarticulation than phrase final vowels, and accented vowels have greater durations and less coarticulation [115]. Introducing phone models that incorporate prosodic state allows such prosody-induced effects to be captured, while retaining the powerful HMM modelling structure and efficient parameter estimation techniques. Computational requirements are increased, though, as a result of the larger parameter count. Also, another disadvantage of this approach is the need for prior labelling of the prosodic phenomena.

The Markov assumption used in HMMs implies a geometric state duration dis- 
tribution specified by the self transition probability. The distribution of phone durations, however, more closely resembles the gamma distribution [116]. This discrepancy has led to modifications to the HMM structure to allow for explicit specification of state durations, such as the expanded state HMM [117] and the hidden semi-Markov model [116]. Including additional duration distributions into model states has been demonstrated to improve recognition accuracy, but at a cost to time performance. The Baum-Welch and Viterbi algorithms used for parameter estimation and decoding rely on the Markov assumption, which is violated by explicit duration modelling. These algorithms then need to be altered to accommodate for this, usually resulting in a loss of efficiency. It has also been argued that the inherent geometric distribution of HMMs is not problematic, as a combination of multiple states sharing the same observation distribution can be used to achieve a broader range of distributions [118].

In an examination of pronunciation variation, Ostendorf et al. [119] found correlations between pronunciation distance, a measure of the difference between the baseform and surface form of phonemes, and prosodic features. The correlation for some F0 and duration features, in particular, were higher than that of trigram probabilities. Using prosodic features in a decision tree trained to predict phonetic surface forms of words in the Switchboard corpus resulted in a small reduction in error rate. Additionally, F0 features were found to have been frequently used in tree questions, even after the inclusion of word-based features. The prosodically conditioned pronunciation model can then be incorporated into an ASR system by supplementing the acoustic features with additional prosodic features, $F$, and calculating:

$$
\begin{aligned}
W^{*} & =\underset{W}{\arg \max } p(W \mid O, F) \\
& =\underset{W}{\arg \max } p(O \mid F, W) p(F \mid W) p(W) \\
& \approx \underset{W, \phi}{\arg \max } p(O \mid \phi, F) p(\phi \mid F, W) p(F \mid W) p(W)
\end{aligned}
$$

where $\phi$ represents the pronunciation sequence. This allows the variation in pronunciation and speaking style, modelled by pitch, energy and duration features, to be combined into the recognition process.

Another use for prosodic information is for post-processing of word hypotheses. An $N$-best or word lattice output of a speech recogniser can be evaluated using a prosodic knowledge source to select those hypotheses which are consistent with the new information. Hirschberg et al. [120] found significant differences between the prosodic patterns of correctly and incorrectly recognised utterances. Adding 
prosodic features to a misrecognition classifier improved prediction of erroneous hypotheses over using ASR-based features alone. The 13\% prediction error obtained by using confidence scores and hypothesised strings was reduced to $11 \%$ after the inclusion of prosody.

Veilleux and Ostendorf [121] used prosody to rescore $N$-best hypotheses by modelling the relationship between labelled prosodic constituents and syntactic parse structure. Parses consisted of part-of-speech information and syntactic bracketing. The probability of an observed sequence of F0, energy and duration measurements, $\mathbf{x}=\left\{x_{1}, \ldots, x_{n}\right\}$, was calculated as:

$$
p(\mathbf{x} \mid \text { parse })=\sum_{\mathbf{a}} p(\mathbf{x} \mid \mathbf{a}) p(\mathbf{a} \mid \text { parse })
$$

where $\mathbf{a}=\left\{a_{1}, \ldots, a_{n}\right\}$ is a sequence of break index and prominence labels. Binary decision trees were used for both the acoustic-prosodic models, $p(\mathbf{x} \mid \mathbf{a})$, and the prosodic-syntactic models, $p(\mathbf{a} \mid$ parse $)$. Tree leaves were associated with probability distributions, which were used to calculate the total prosodic probability of a given parse. Linear combinations of the prosodic scores with acoustic and language model scores obtained from the recogniser were then used to reorder an $\mathrm{N}$-best list. For utterances in which the correct hypothesis was present in the top ten hypotheses, this rescoring approach resulted in a relative $23 \%$ improvement in the average rank of the correct hypothesis.

Batliner and Nöth [122] describe the use of prosodic information in the VERBMOBIL speech-to-speech translation system. The extraction of prosodic features in this approach is based on the output of a speech recogniser. F0, energy and duration features are extracted from regions demarcated by hypothesised word boundaries. This allows for the mapping of prosody onto units that are directly usable by subsequent processing modules. The inputs to the prosody module are the speech signal and a word hypotheses graph (WHG), and the output is a word graph annotated with various prosodic phenomena such as accents and boundaries. A set of 95 prosodic features plus an additional 30 part-of-speech (POS) features are used in this system, calculated at context sizes of up to \pm 2 words. Because the same feature may be calculated for multiple contexts, there are highly correlated features in the resulting set. These features are used directly in a multi layer perceptron to classify the prosodic events. Therefore, in this approach, the statistical classifier is relied upon to determine the appropriate relationships between extracted features and the prosodic classes.

Using a very large feature set can be computationally costly and can also increase 
the potential for over-training. If there are strong correlations between the features, then it is possible to apply dimensionality reduction techniques to reduce the number of features with minimal loss of information. Batliner et al. [123] applied linear discriminant analysis (LDA) to reduce a set of prosodic features used for classifying word accents and boundaries. The 276 original features were reduced to 6 features for accent classification and 11 features for boundary classification, with a performance degradation of $2-3 \%$.

Annotating a WHG with prosodic information has a number of benefits. Firstly, WHGs are a commonly used data structure and it is simple to extract the relevant prosodic information from them. Aligning prosodic features with words is also much cheaper computationally than aligning with the underlying phonemes. While the phone sequence is calculated by the decoder in the recognition process, it is normally not accessible to subsequent processing steps. Performing forced alignment on the output to recover the HMM state sequence can be a very slow process. Phonetic alignments can be useful, however. Syllabic units, which are the units primarily associated with accentuation, can be determined more reliably from segmental information than from word information. Pause durations, too, can be easily obtained from silence models.

A general framework for incorporating prosodic information into ASR systems is presented in Shriberg and Stolcke [111]. An important aspect of this approach is that explicit identification of phonological constituents is not used. Rather, stochastic methods are employed to learn relations between acoustic observations and linguistic entities. The inclusion of a prosodic feature stream, $F$, into the ASR equation gives:

$$
\begin{aligned}
W^{*} & =\underset{W}{\arg \max } p(W \mid O, F) \\
& =\underset{W}{\arg \max } p(W) p(O, F \mid W) \\
& \approx \underset{W}{\arg \max } p(W) p(O \mid F, W) p(F \mid W)
\end{aligned}
$$

$p(W)$ is a standard language model. The acoustic model, $p(O \mid F, W)$ can be additionally conditioned on prosody. If it is assumed that the acoustic and prosodic features are independent given the word sequence, then this can be reduced to a standard, prosody-independent acoustic model $p(O \mid W) \cdot p(F \mid W)$ is used to model the interaction between prosodic features and word identities. For example, Gadde [124] used this approach to incorporate duration features into a recogniser. Each word was associated with a vector of phone durations, modelled by Gaussian mixture models (GMMs). Durations of $N$-best list hypotheses were obtained by forced alignment, and these were rescored using a combined score from the acoustic, language and 
duration models. Reductions in WER of up to $1 \%$ were obtained on two test sets using this duration model rescoring method.

The previous modelling approach can be further extended by modelling the interaction of prosody with linguistic structures above the word level. Instead of estimating $p(F \mid W)$ directly, it is modelled with respect to an intermediate linguistic structure, $S$, giving:

$$
\begin{aligned}
W^{*} & =\underset{W}{\arg \max } p(W) p(O \mid W) p(F \mid W) \\
& =\underset{W}{\arg \max } p(W) p(O \mid W) \sum_{S} p(F \mid S, W) p(S \mid W)
\end{aligned}
$$

$p(F \mid S, W)$ models the relationship between prosody and linguistic structure, and $p(S \mid W)$ models the relationship between the structures and the word sequence. These components are trained on data labelled with the linguistic structures, which are simpler to annotate than perceptual prosodic classes. There is also no need to train these models on the same data as the acoustic or language models, giving them a high level of reusability for different tasks.

An instance of this modelling approach is that of Stolcke et al. [125]. The linguistic structures used, called hidden events, included boundaries, pauses and disfluencies. An $n$-gram model was used to model the sequence of words and structures $p(W, S)$. Posterior probabilities $p(S \mid F, W)$ were modelled by a decision tree, and these probabilities were used to calculate $p(F \mid S, W)$. By assuming that prosodic features correlate locally with structural events $S=\left\{E_{1}, \ldots, E_{n}\right\}$ located at word boundaries, $p(F \mid S, W)$ can be defined as:

$$
p(F \mid S, W) \approx \prod_{i-1}^{n} p\left(F_{i} \mid E_{i}, W\right)
$$

These were used as the emission probabilities of an HMM modelling $p(F, S, W)$. States of this HMM correspond to $(W, S)$ pairs, and state transitions were determined by $p(W, S)$. The summation in Equation (3.3) can be efficiently calculated using the forward algorithm for HMMs. Rescoring 100-best lists with the prosodic models resulted in a $0.9 \%$ absolute reduction in WER compared to a trigram model.

Chen and Hasegawa-Johnson [126] also described an approach for modelling the interaction between words, syntax, and prosody in a language model. Word-prosody pairs, $\left(w_{i}, p_{i}\right)$, were modelled in a factored bigram. The prosodic factor consisted of ToBI pitch accent and break index labels, each simplified to a binary value. Because the introduction of a prosodic variable into the word identity greatly fragments the 
data, the estimation of probabilities were further conditioned on POS categories. The prosody dependent word bigram probabilities were approximated as:

$$
\begin{aligned}
p\left(w_{j}, p_{j} \mid w_{i}, p_{i}\right) & =\sum_{c_{i}, c_{j}} p\left(p_{j} \mid c_{j}, c_{i}, w_{j}, w_{i}, p_{i}\right) p\left(c_{j}, c_{i} \mid w_{j}, w_{i}, p_{i}\right) p\left(w_{j} \mid w_{i}, p_{i}\right) \\
& \approx \sum_{c_{i}, c_{j}} p\left(p_{j} \mid c_{j}, c_{i}, p_{i}\right) p\left(c_{j}, c_{i} \mid w_{j}, w_{i}\right) p\left(w_{j} \mid w_{i}, p_{i}\right)
\end{aligned}
$$

where $c_{i}$ is the POS label of word $w_{i}$. The approximation:

$$
p\left(p_{j} \mid c_{j}, c_{i}, w_{j}, w_{i}, p_{i}\right) \approx p\left(p_{j}, c_{j}, c_{i}, p_{i}\right)
$$

assumes that, given the grammatical context, prosodic labels are independent of word identities. This allows for robust estimation of model parameters, as the number of POS and prosody labels are small in comparison to the number of words. This language model, when combined with acoustic models allowing for prosodyinduced allophonic variation, reduced the WER on a corpus of read speech by $2.2 \%$ compared to a standard back-off bigram model.

\subsection{Prosodic Knowledge for Language Modelling}

The inclusion of prosodic knowledge has been demonstrated to be able to improve continuous speech recognition performance. Prosodic features, however, are inherently different from the spectral features used by recognisers to determine phonetic identity. While some aspects of prosody affect the spectral characteristics of speech, a large component of prosody is predominantly temporal in nature. The time scale on which prosody operates is also both longer and more variable. Accentuation, for instance, affects syllables, while intonational effects such as those relating to speaker attitudes can span utterances and dialogues. While such long span phenomena may not have a clearly defined effect on the underlying word sequence, they are not completely independent. An example of this is accentuation, which can be impacted on by dialogue structure and focus.

Incorporating prosodic information into a speech recogniser therefore requires modelling the interaction between prosody and linguistic units of varying scope. A language model would appear to be a suitable choice for this. Indeed, some of the work discussed in the previous section has made effective use of prosodic information in language models. Language models have the advantage of being able to describe a wide range of phenomena, as exemplified by the hidden event modelling approach. 
In our work, we intend to further explore some areas not fully covered by the previous research. In particular, work such as that of Gadde [124] and Chen et al. [126] have concentrated on the use of a limited set of features, such as duration and F0 only, and modelled them with approaches specific to those features. We will attempt to use a broader range of features, and incorporate them in a more general framework. We plan to do this using the maximum entropy model, which provides a consistent method for including multiple and varied sources of knowledge. Using this model also allows us to further investigate the correlations between prosody and syntax, and use this information without being restricted by specific modelling constraints. The theory behind maximum entropy modelling will be detailed in the next chapter.

From a system design perspective, combining the prosodic knowledge into the language model maintains simplicity and modularity. A language model can be developed and optimised independently of the acoustic models, which can provide significant time benefits. Language models also have numerous applications besides ASR, and a modular approach allows them to be easily adapted for other tasks. The counterpoint to this is that the separation of the acoustic and language models can restrict the level of interaction between them. It is possible that fully exploiting some prosodic effects requires tightly integrated modelling of both segmental and suprasegmental properties. In particular, language models are not designed to model sub-word phenomena. Therefore, the effects of prosody on phonetic variation are difficult to capture. This is only one of many realisations of prosody, though, and the majority of prosodic features are suprasegmental. This, in combination with the development and computational advantages, means the language model is still a useful medium for integration of prosodic knowledge. 


\section{Chapter 4}

\section{Maximum Entropy}

Many NLP problems can be formulated as a statistical classification problem in which a probability distribution must be learned from a sample of data. An empirical sample can only provide an incomplete representation of the true distribution, and there are many possible distributions which can be consistent with the data. Given a number of competing models, it might be reasonable to seek justification for preferring one particular form of distribution over another. Of those models which are computationally tractable, an intuitive choice might be the one which makes the fewest assumptions about the unobserved aspects of the data. This is the idea underlining the maximum entropy model, which makes use of information theory to formalise the concept of minimal assumptions.

This chapter will begin by introducing the principle of maximum entropy. A simple example is used to highlight the main ideas behind this method. The second section will describe how knowledge is represented in the maximum entropy framework. Following this, the concepts will be formalised into a mathematical model. The third section will discuss the parameter estimation problem, and how this can be tackled using iterative scaling algorithms tailored for solving maximum entropy models, as well as more general constrained optimisation techniques. The data sparsity issue will be addressed in the fourth section, and it will describe some techniques which can be used to smooth parameter estimates in these models. The final section in this chapter will discuss how different types of information can be effectively combined under the maximum entropy framework.

\subsection{Maximum Entropy Principle}

A simple example will be used to illustrate the principle of maximum entropy. Consider the scenario of guessing the suit of a card, drawn from a standard deck of 52 . 
Let $s \in\{\boldsymbol{s}, \diamond, \varnothing, \mathbf{p}\}$ be the suit of the card. If no other information is provided, a good choice for the distribution of $s$ is:

\begin{tabular}{ccccc}
\hline$s$ & as & $\diamond$ & $\varnothing$ & ค \\
\hline$p(s)$ & $1 / 4$ & $1 / 4$ & $1 / 4$ & $1 / 4$ \\
\hline
\end{tabular}

This distribution can be arrived at by assuming that every card in the deck has an equal chance of being chosen. Now, assume that the original deck is no longer standard. Instead, the 52 cards are composed of 10 clubs, and an unknown quantity of each of the three remaining suits. The previous estimate of the distribution is not consistent with this new information, so a new distribution should be chosen. There are numerous solutions which satisfy the requirement. For example:

\begin{tabular}{ccccc}
\hline$s$ & $\boldsymbol{\phi}$ & $\diamond$ & $\varnothing$ & $\boldsymbol{\phi}$ \\
\hline$p(s)$ & $10 / 52$ & 0 & $21 / 52$ & $21 / 52$ \\
\hline
\end{tabular}

is a valid solution. Of course, a distribution such as this is hard to justify, and would require making some unfounded assumptions about the organisation of the other cards. Intuitively, a much more reasonable solution would be:

\begin{tabular}{ccccc}
\hline$s$ & $\boldsymbol{\phi}$ & $\diamond$ & $\varnothing$ & A \\
\hline$p(s)$ & $10 / 52$ & $14 / 52$ & $14 / 52$ & $14 / 52$ \\
\hline
\end{tabular}

This remains consistent with the known facts but, unlike the previous solution, avoids making any guesses about unknown information. It does so by assigning the most uniform distribution possible without violating the given constraints. This is known as the maximum entropy estimate, and it is the solution which is "maximally noncommittal with regard to missing information" [127]. The maximum entropy principle can be applied to problems for which only partial evidence is available. Such under-specification is very common in NLP tasks, in which models are developed from samples providing only an incomplete representation of the true distribution to be modelled.

For a basic scenario such as the one described here, obtaining the maximum entropy solution is trivial. As the number of constraints grows, though, the problem quickly becomes difficult to solve analytically. The formalisation of some intuitions used in this example, and the mathematical framework for solving more general problems, will be presented in the next section. 


\subsection{Maximum Entropy Models}

\subsubsection{Representing knowledge}

In the example described previously, the task was to estimate the distribution of $s$. When no additional information was given, $s$ was assumed to have uniform distribution. When a fact about $s$ was provided, a new distribution was derived, somewhat intuitively, which was consistent with that fact. Under the maximum entropy framework, these known facts are formulated as a set of feature functions, or more simply, features. Feature functions are commonly binary valued, although real valued features are also possible. In the example, something is known about the number of clubs in the deck. Therefore, the following feature function can be used to encode this knowledge:

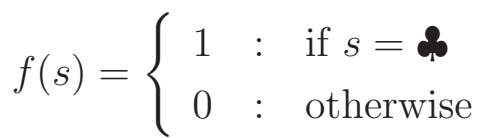

This is an indicator function that is active whenever $s=\boldsymbol{\$}$. To use the knowledge that $p(s)=\frac{10}{52}$, the expected value of $f$ with respect to the model, $p$, can be set to equal the desired target:

$$
\mathbb{E}_{p}[f]=\frac{10}{52}
$$

This constraint equation imposes a linear constraint on the solution in the space of all possible models. If more than one constraint equation exists, their intersection defines the feasible region in which the solution lies. This is the subset of models which satisfy the specified constraints. So long as there are no inconsistent constraints, this subset will be non-empty, and a (possibly non-unique) solution will exist.

\subsubsection{Conditional models}

The previous example dealt with an unconditional probability distribution, $p(s)$. In many NLP problems, it is often necessary to find conditional probabilities. Generally, the requirement is to estimate a class label $w \in W$ given some some observed context $h \in H$. In an $n$-gram language model, for example, $W$ would represent the set of words in the vocabulary, and $H$ the set of $(n-1)$ length word histories. A binary feature in a conditional model, then, has the form:

$$
f(h, w)= \begin{cases}1 & : \text { if }(h, w) \in X \\ 0 & : \text { otherwise }\end{cases}
$$


where $X$ is an arbitrary subset of the event space $H \times W$. The purpose of the feature is to identify co-occurrences of a given target and context pair. The specific identity of a feature is dependent on the task required, and must be provided as an input to the modelling process.

As done in the previous example, incorporating knowledge about a problem into the model is done by equating the model's feature expectations to target values using constraint equations. In language modelling applications, where a large sample of data is usually available, target values can be determined empirically. For a sample of $n$ observations, $\left(h_{1}, w_{1}\right),\left(h_{2}, w_{2}\right), \ldots,\left(h_{n}, w_{n}\right)$, the expected value of a feature $f(h, w)$ with respect to the empirical distribution $\tilde{p}(h, w)$ is:

$$
\mathbb{E}_{\tilde{p}}[f]=\sum_{h, w} \tilde{p}(h, w) f(h, w)
$$

where $\tilde{p}(h, w)=\frac{c(h, w)}{n}$ and $c(h, w)$ is the number of times $(h, w)$ occurs in the sample.

The expectation of feature $f(h, w)$ with respect to a conditional model $p(w \mid h)$ is given by:

$$
\mathbb{E}_{p}[f]=\sum_{h, w} \tilde{p}(h) p(w \mid h) f(h, w)
$$

Equation (4.3) does not calculate feature expectations with respect to the true model distribution $p(h, w)$. Because the space of contexts, $H$, can be very large, a summation over the event space may be intractable. The approximation is therefore made to circumvent the need for this. Although it is unlikely that $p(h) p(w \mid h)$ can be accurately approximated by $\tilde{p}(h) p(w \mid h)$, this is not actually necessary. Typically, for any given feature $f_{i}, f_{i}(h, w)$ evaluates to zero for the large majority of $(h, w)$ pairs. Subsequently, the two distributions need to be close only for the cases when $f_{i}(h, w)$ is non-zero, which is a more reasonable assumption.

Combining Equation (4.2) and Equation (4.3) gives the final form of the constraint equation for conditional models:

$$
\sum_{h, w} \tilde{p}(h) p(w \mid h) f(h, w)=\sum_{h, w} \tilde{p}(h, w) f(h, w)
$$

A distinguishing characteristic of the maximum entropy model is that it is maximally uniform given the constraints. Uniformity, in this case, is quantified in terms of the model's conditional entropy:

$$
H(p)=-\sum_{h, w} \tilde{p}(h) p(w \mid h) \log p(w \mid h)
$$


Therefore, of the set of all models which are consistent with the constraints, $\mathcal{P}$, the one with the maximal entropy, $p^{*}$, is:

$$
p^{*}=\underset{p \in \mathcal{P}}{\arg \max } H(p)
$$

subject to $k$ constraints of the form of Equation (4.4), and the implicit constraint that $p(w \mid h)$ describes a probability distribution:

$$
\sum_{w} p(w \mid h)=1
$$

This is a problem in constrained optimisation, and Equation (4.5) is referred to as the primal problem. Introducing a Lagrange multiplier, $\lambda_{i}$, for each of the $k+1$ constraints gives the Lagrangian function:

$$
\mathcal{L}(p, \lambda)=H(p)+\sum_{i=1}^{k} \lambda_{i}\left(\mathbb{E}_{p}\left[f_{i}\right]-\mathbb{E}_{\tilde{p}}\left[f_{i}\right]\right)+\lambda_{0}\left(\sum_{w} p(w \mid h)-1\right)
$$

Setting the derivative of $\mathcal{L}(p, \lambda)$, with respect to the primal variable $p(w \mid h)$, to zero:

$$
\frac{\partial}{\partial p(w \mid h)} L(p, \lambda)=\tilde{p}(x)\left(-\log p(w \mid h)-1+\sum_{i=1}^{k} \lambda_{i} f_{i}(h, w)+\lambda_{0}\right)=0
$$

and substituting the result back into Equation (4.6) gives the parametric form of $p(w \mid h)$ :

$$
\begin{aligned}
p^{*}(w \mid h) & =\frac{1}{Z(h)} \exp \left(\sum_{i=1}^{k} \lambda_{i} f_{i}(h, w)\right) \\
Z(h) & =\sum_{w} \exp \left(\sum_{i=1}^{k} \lambda_{i} f_{i}(h, w)\right)
\end{aligned}
$$

$Z(h)$ is a normalisation term ensuring $\sum_{h, w} p(w \mid h)=1$, and is called the partition function. The value of the Lagrangian when $p=p^{*}$ :

$$
\mathcal{L}\left(p^{*}, \lambda\right)=\sum_{h} \tilde{p}(h) \log Z(h)-\sum_{i=1}^{k} \lambda_{i} \mathbb{E}_{\tilde{p}}\left[f_{i}\right]
$$

is the dual function. This satisfies the Karush-Kuhn-Tucker optimality conditions [128], and the original primal problem given by Equation (4.5) is concave. Therefore, strong duality holds and the solution to the entropy maximisation problem of 
Equation (4.5) is equivalent to the solution of the dual minimisation problem:

$$
\underset{p \in \mathcal{P}}{\arg \max } H(p)=\underset{\lambda}{\arg \min } \mathcal{L}\left(p^{*}, \lambda\right)
$$

Solving the problem in the domain of the dual has two advantages. Firstly, it involves unconstrained optimisation of a convex function. Therefore, any local minimum is also the global minimum. Secondly, its parameters are the set of $k$ real-valued variables $\Lambda=\left\{\lambda_{1}, \ldots, \lambda_{k}\right\}$, while the dimension of the primal problem is determined by the distribution's number of degrees of freedom, and may be unbounded.

The maximum entropy solution also has another property that is desirable in statistical modelling. The log-likelihood of a set of samples of $(h, w)$, given a conditional probability distribution with the form of Equation (4.7), is:

$$
\begin{aligned}
L(\Lambda) & =\sum_{h, w} \tilde{p}(h, w) \log p(w \mid h) \\
& =\sum_{h, w} \tilde{p}(h, w) \sum_{i} \lambda_{i} f_{i}(h, w)-\sum_{h} \tilde{p}(h) \log Z(h) \\
& =-\mathcal{L}\left(p^{*}, \lambda\right)
\end{aligned}
$$

The log-likelihood is the negative of Equation (4.9), and therefore the set of Lagrange multipliers, $\Lambda$, that minimises the dual function also specifies the model, amongst the family of exponential models, that maximises the likelihood of the empirical data. This, in conjunction with the minimal bias property, provides additional motivation for the use of the maximum entropy model.

\subsubsection{Minimum divergence}

Another interpretation of entropy maximisation is to consider it as a minimisation of the distance between two probability distributions. The Kullback-Leibler (KL) divergence [129] between the model distribution, $p(x)$, and a reference distribution [130], $q(x)$, is given by:

$$
D(p \| q)=\sum_{x} p(x) \log \frac{p(x)}{q(x)}
$$

If $q(x)$ is the uniform distribution, then $D(p \| q)=-H(p)$, and therefore, minimising the $\mathrm{KL}$ divergence is equivalent to maximising the entropy function of $p$. Minimising $D(p \| q)$ when $q$ is not uniform gives the Minimum Discrimination Information (MDI) solution that incorporates the knowledge contained in $q$. This form of the model is particularly useful when $q$ is large, or represents information which is difficult 
to formulate as maximum entropy features. In language modelling, for example, $n$ gram models can encode a large amount of information in a faster and more scalable manner than maximum entropy models. The MDI solution is to use the $n$-gram as a reference distribution, resulting in the following model:

$$
\begin{aligned}
p^{*}(w \mid h) & =\frac{q(w \mid h)}{Z(h)} \exp \left(\sum_{i=1}^{k} \lambda_{i} f_{i}(h, w)\right) \\
Z(h) & =\sum_{w} q(w \mid h) \exp \left(\sum_{i=1}^{k} \lambda_{i} f_{i}(h, w)\right)
\end{aligned}
$$

\subsection{Parameter Estimation}

Solving the dual problem requires finding the vector of parameters, $\Lambda$, which minimises the dual function, or equivalently, maximises the log-likelihood. The objective (log-likelihood) function is concave in $\Lambda$, so it has a global maximum where its gradient is zero. In general, this has no closed form solution, so the problem is solved numerically. If a model is initialised with an arbitrary set of parameters, $\Lambda$, and a new set of parameters:

$$
\Lambda+\Delta=\left\{\lambda_{1}+\delta_{i}, \ldots, \lambda_{k}+\delta_{k}\right\}
$$

can be chosen such that the log-likelihood of the model with the updated parameters does not decrease, then this process can be iterated until the maximum is reached. An additional terminating condition may also be specified, such as when the gain in log-likelihood falls below a given threshold.

\subsubsection{Iterative scaling}

The Generalized Iterative Scaling (GIS) [131] algorithm is one method for calculating the update values. In order to guarantee convergence, its is required that $\sum_{i=1}^{k} f_{i}(h, w)=f^{\#}$ for all $(h, w)$, where $f^{\#}$ is a constant. This condition can be enforced through the use of a correction feature:

$$
\begin{aligned}
f^{\#} & =\max _{h, w} \sum_{i=1}^{k} f_{i}(h, w) \\
f_{k+1}(h, w) & =f^{\#}-\sum_{i=1}^{k} f_{i}(h, w)
\end{aligned}
$$


At the point of convergence, the constraints of the problem are satisfied. That is, $\mathbb{E}_{p}\left[f_{i}\right]=\mathbb{E}_{\tilde{p}}\left[f_{i}\right]$ for all $i$. Under GIS, updates are calculated based on the ratio of these two expectations:

$$
\delta_{i}=\frac{1}{f^{\#}} \log \left(\frac{\mathbb{E}_{\tilde{p}}\left[f_{i}\right]}{\mathbb{E}_{p}\left[f_{i}\right]}\right)
$$

Each $\delta_{i}$ is calculated independently of the others, so the scaling of the update by $\frac{1}{f \#}$ is performed to avoid overshooting of parameters due to feature interactions. However, this also has the effect of slowing the convergence rate, since the step size is inversely proportional to $f^{\#}$. By slowing all parameter updates to match the worst case, the number of iterations required to converge is significantly increased.

The Improved Iterative Scaling (IIS) [132] algorithm does not require the sum of features over the set of samples to be a constant, and therefore does not need the correction feature. It can be used, and is guaranteed to converge, for the case when all feature functions are non-negative. IIS uses a lower bound on the change in log-likelihood, $\Delta L(\Lambda)$ :

$$
\begin{aligned}
\Delta L(\Lambda) \geq & \sum_{h, w} \tilde{p}(h, w) \sum_{i=1}^{k} \delta_{i} f_{i}(h, w)+1- \\
& \sum_{h} \tilde{p}(h) \sum_{w} p(w \mid h) \sum_{i=1}^{k} \frac{f_{i}(h, w)}{f^{\#}(h, w)} \exp \left(\delta_{i} f^{\#}(h, w)\right)
\end{aligned}
$$

where $f^{\#}(h, w)=\sum_{i=1}^{k} f_{i}(h, w)$. The updates in IIS are then computed by maximising this lower bound function with respect to each $\lambda_{i}$. They are found as the solutions of:

$$
\sum_{h, w} \tilde{p}(x) p(w \mid h) f_{i}(h, w) \exp \left(\delta_{i} f^{\#}(h, w)\right)=\sum_{h, w} \tilde{p}(h, w) f_{i}(h, w)
$$

and can be efficiently calculated using Newton's method. IIS generally converges in fewer iterations than GIS; however, the computation cost at each iteration is also higher.

A tighter lower bound is used in the Faster Iterative Scaling (FIS) algorithm [133] by not completely decoupling the correlations between the $\delta_{i}$ 's:

$$
\begin{aligned}
\Delta L(\Lambda) \geq & \sum_{h, w} \tilde{p}(h, w) \sum_{i} \delta_{i} f_{i}(h, w)- \\
& \sum_{h} \tilde{p}(x) \sum_{i} \frac{f_{i}(h, w)}{f^{\#}(h, w)} \log \left(\sum_{w} p(w \mid h) \exp \left(\delta_{i} f^{\#}(h, w)\right)\right)
\end{aligned}
$$


The tighter bound improves the rate of converge of this algorithm over IIS, but there is greater computational complexity in calculating this function at each iteration. FIS was found to perform favourably in comparison to IIS on several small text classification tasks [133].

\subsubsection{Gradient based methods}

The iterative scaling algorithms do not make use of the objective function's gradient. Incorporating gradient information into the search can lead to faster convergence, but it is traded off against the cost of computing the gradient, $g(\Lambda)$. In the case of the log-likelihood function, though:

$$
\begin{aligned}
\frac{\partial}{\partial \lambda_{i}} L(\Lambda) & =\sum_{h, w} \tilde{p}(h, w) f_{i}(h, w)-\sum_{h, w} \tilde{p}(x) p(w \mid h) f_{i}(h, w) \\
& =\mathbb{E}_{\tilde{p}}\left[f_{i}\right]-\mathbb{E}_{p}\left[f_{i}\right]
\end{aligned}
$$

The derivative with respect to $\lambda_{i}$ is simply the difference between the model and empirical feature expectations. These are already calculated in iterative scaling, so there is minimal overhead associated with using the gradient. The model parameters can then be updated in the direction of steepest ascent, by setting updates as:

$$
\Delta=-\alpha \mathbf{g}(\Lambda)
$$

for some step size $\alpha$. The search direction at each iteration is orthogonal to that of the previous iteration. This results in a zig-zag path to the solution, and the same direction can often be taken at different iterations. If the gradient of the function varies greatly in different directions, the steepest ascent method may take many iterations to converge. It is also dependent on the initial search values. An improved choice of update direction is given by the conjugate gradient (CG) method [134]. The initial search direction is identical to that of steepest ascent. Subsequent search directions, though, are chosen such that they are conjugate rather than orthogonal. In this approach, each search direction is only considered once, and can converge faster than steepest ascent.

The log-likelihood function of an exponential model has a well defined second derivative, and the convergence performance of an optimisation algorithm can be further improved by the use of second order gradients. Newton's method, for example, uses the update rule:

$$
\Delta=H^{-1}(\Lambda) \mathbf{g}(\Lambda)
$$


where $H(\Lambda)$ is the hessian matrix of $L(\Lambda)$. Because calculating $H(\Lambda)$ and its inverse is computationally expensive, quasi-Newton methods such as BFGS use a local approximation of the inverse hessian. The hessian is a symmetric $k \times k$ matrix where $k$ is the number of variables of the objective function. For large problems, storing the hessian is not practical. The limited memory BFGS [135] method reduces the space requirements by generating matrices using information from previous iterations.

Malouf [136] compared GIS, IIS, steepest ascent, CG and BFGS algorithms on NLP classification tasks. The iterative scaling methods were found to perform quite poorly, while BFGS outperformed the other algorithms in all experiments. Similar results were obtained by Minka [137] in a comparison of optimisation algorithms for logistic regression. The CG and BFGS methods both performed well, with especially good results obtained by CG when features were uncorrelated.

\subsection{Smoothing}

As was shown in Section 4.2, the maximum entropy solution is also the maximum likelihood solution of the family of models having exponential form. Like other maximum likelihood models, they are subject to over-training, especially when large numbers of features are used. Therefore, some form of smoothing is required to obtain robust estimates. For the language modelling problem, one option is to apply standard $n$-gram discounting techniques, such as those described in Section 2.1.2, to the data before fitting the model. The expectations of model features can then be constrained to their discounted, rather than true averages over the training data. For the maximum entropy model, though, there are other more general approaches which can be used to achieve the same goal.

\subsubsection{Feature selection}

One of the objectives of smoothing is to assign some probability mass to events that have not occurred in training. There is, however, a notable difference between how maximum entropy models and $n$-gram models handle unseen events. Consider a maximum entropy model using only bigram features. If we assume the feature context to be the previous word only, $h=w_{i-1}$, then for every bigram in the training data, $(x, y)$, a feature of the following form is defined:

$$
f_{x, y}(h, w)= \begin{cases}1 & : \text { if } h=x \text { and } w=y \\ 0 & : \quad \text { otherwise }\end{cases}
$$


Expectations of the features are constrained to equal their empirical values according to Equation (4.4). Now, assume that during testing, a particular bigram was encountered, say Monday evening, which did not appear in training. An unsmoothed bigram model trained on the same data would assign $p_{\mathrm{bg}}($ evening $\mid$ Monday $)=0$. In the maximum entropy model, $f_{x, y}($ Monday, evening $)=0$ for all features $f_{x, y}$. The model probability is then:

$$
p_{\text {me }}(\text { evening } \mid \text { Monday })=\frac{1}{Z(\text { Monday })}
$$

where $Z$ (Monday) is the normalising term calculated over all bigrams beginning with the word Monday. As a result, $p_{\text {me }} \neq 0$. If Monday also did not occur in the training data, the probability becomes a uniform distribution based on the size of the vocabulary, $|W|$ :

$$
p_{\text {me }}(\text { evening } \mid \text { Monday })=\frac{1}{|W|}
$$

Therefore, a limited degree of smoothing can be achieved simply by not constraining a feature. This allows the problem of over-training to be approached from the point of view of feature selection.

The most basic application of this is to use count cutoffs to eliminate rare features. It avoids over-fitting the model to potentially unreliable parts of the training data. It also benefits the model by reducing the number of parameters. Ratnaparkhi [138] achieved good performance in several NLP classification tasks by using count cutoffs. However, a problem with this naive approach is that it makes no distinction between features having low counts because they are under-represented by the data and those which truly represent uncommon information. Discarding all such features inevitably results in the loss of some useful knowledge. More sophisticated feature selection algorithms incorporate features into the model based on their predictive power. Berger et al. [139] presented a greedy, incremental algorithm for selecting a subset from a large set of potential features. An approximated loglikelihood gain was used to measure the contribution of a candidate feature. The feature providing the greatest increase in likelihood was then added to the model's feature set, and the process repeated. Della Pietra [132] proposed a similar algorithm using an information gain criterion.

\subsubsection{Regularisation}

Relying solely on the uniform property of maximum entropy models for smoothing tends to be inadequate, as it only provides very weak smoothing. If the model 
parameters $\Lambda$ are very large, some events may still receive near-zero probabilities. Another problem can occur with explicit modelling of a zero-count feature. If a particular feature does not occur in the training data, its parameter tends towards negative infinity. For any subsequent event where that feature is active, the probability assigned by the model is zero. In order to sufficiently smooth a model trained on sparse data, further smoothing techniques are still required.

Chen and Rosenfeld [140] described the application of a zero mean Gaussian prior distribution on the model parameters. Rather than performing maximum likelihood estimation, maximum a posterior (MAP) estimation is performed to find the parameters that maximise the modified objective function:

$$
L^{\prime}(\Lambda)=L(\Lambda)-\sum_{i=1}^{k} \frac{\lambda_{i}^{2}}{2 \sigma_{i}^{2}}
$$

where $\sigma^{2}$ is the variance of the prior. The second term in this equation is a weighted $l_{2}$ norm of the parameters, and assists smoothing by penalising models which diverge from the uniform distribution. Under this regularised model, feature expectations are no longer constrained to equal their empirical expectations. Instead, they are discounted such that:

$$
\mathbb{E}_{p}\left[f_{i}\right]=\mathbb{E}_{\tilde{p}}\left[f_{i}\right]-\frac{\lambda_{i}}{\sigma_{i}^{2}}
$$

This results in model expectations that are generally lower than their empirical targets, which is reasonable given that the empirical values tend to overestimate probabilities of observed events. Chen and Rosenfeld found that a maximum entropy model with Gaussian prior smoothing performed competitively with modified a Kneser-Ney smoothed $n$-gram. These two approaches consistently outperformed other $n$-gram smoothing methods, as well as a maximum entropy model smoothed with Katz smoothing.

Placing a Gaussian prior over the model parameters is equivalent to constraining the feature expectations to a discounted empirical value. Kazama and Tsujii [141] proposed an inequality model in which features were constrained to lie within a given range. This was formulated as an entropy maximisation problem subject to the two inequality constraints:

$$
\begin{aligned}
& \mathbb{E}_{\tilde{p}}\left[f_{i}\right]-\mathbb{E}_{p}\left[f_{i}\right]-A_{i} \leq 0 \\
& \mathbb{E}_{p}\left[f_{i}\right]-\mathbb{E}_{\tilde{p}}\left[f_{i}\right]-B_{i} \leq 0
\end{aligned}
$$

for $A_{i}, B_{i}>0$, representing upper and lower bounds, respectively. If there is a solu- 
tion to this problem at the limits of these inequalities, corresponding to a standard maximum entropy model, then a solution to the inequality model is also guaranteed to exist. The regularised log-likelihood function to maximise is:

$$
L^{\prime}(\alpha, \beta)=L(\alpha, \beta)-\sum_{i} \alpha_{i} A_{i}-\sum_{i} \beta_{i} B_{i}
$$

This is equivalent to the log-likelihood of a maximum entropy model penalised by a weighted $l_{1}$ norm, or exponential prior. In some cases, this prior more closely resembles the actual model parameter distribution than the Gaussian prior [142]. At the optimal point, the model satisfies the conditions:

$$
\begin{aligned}
\alpha_{i}\left(\mathbb{E}_{\tilde{p}}\left[f_{i}\right]-\mathbb{E}_{p}\left[f_{i}\right]-A_{i}\right) & =0 \\
\beta_{i}\left(\mathbb{E}_{p}\left[f_{i}\right]-\mathbb{E}_{\tilde{p}}\left[f_{i}\right]-B_{i}\right) & =0
\end{aligned}
$$

If $\alpha_{i}>0$ or $\beta_{i}>0$, then $\mathbb{E}_{\tilde{p}}\left[f_{i}\right]-\mathbb{E}_{p}\left[f_{i}\right]=A_{i}$ or $-B_{i}$. For these cases, model expectations are constrained to equal the empirical expectation minus a constant. This is effectively absolute discounting, which has been shown to work very well for language modelling tasks [143].

A property of the exponential prior is that it tends to favour parameters which are exactly zero. When this is the case, $-B_{i}<\mathbb{E}_{\tilde{p}}\left[f_{i}\right]-\mathbb{E}_{p}\left[f_{i}\right]<A_{i}$, and the optimal point lies strictly within the feasible region defined by constraining feature $f_{i}$. The removal of this feature, then, does not alter the solution. This provides an implicit form of feature selection which is not present with the Gaussian prior. The amount of smoothing, and the threshold for pruning features, then, is controlled by the parameters $A_{i}$ and $B_{i}$. The most basic method for deriving these is to use a fixed width, $W$, for all parameters such that:

$$
A_{i}=-B_{i}=\frac{W}{n}
$$

where $n$ is the number of training samples. A number of more sophisticated techniques are evaluated in [141], though none significantly outperformed this simple approach.

Evaluations of the inequality model have found it to perform slightly better than models using a Gaussian prior in two text categorisation experiments. It also has the advantage that fewer features are required. However, the presence of both upper and lower bounds on feature expectations results in a doubling of the parameter space. This leads to a significant increase in time requirements during parameter 
estimation.

\subsection{Combining Knowledge Sources}

The maximum entropy model has a number of useful properties. It provides the least biased estimate given incomplete information. It is also the maximum likelihood model of the exponential family. Parameter estimation is independent of the modelling task, and efficient parameter estimation techniques exist for training the model. Perhaps its greatest advantage, though, is its flexibility in modelling phenomena. It provides a principled and consistent framework for encoding knowledge. The feature function representation of information, used to capture co-occurrences between contexts and target classes, is extremely general. Any property which can be expressed as statistics of context and target pairs can subsequently be included into the model, irrespective of what information it contains.

Features are incorporated into the maximum entropy model using the constraint equations of the form of Equation (4.4). These constraints can be considered to be weak in the sense that they only constrain the features' expectations. Consider a corpus in which the word, evening, and word bigram Monday evening, occur with probabilities $\tilde{p}$ (evening) and $\tilde{p}$ (Monday evening) respectively. These are also the probabilities assigned by an unsmoothed bigram model (i.e. $p_{\mathrm{bg}}($ evening $)=\tilde{p}$ (evening) and $p_{\text {bg }}($ Monday evening $)=\tilde{p}($ Monday evening $)$. If the word sequence Monday evening was observed frequently in the training data, then $p_{\mathrm{bg}}$ (Monday evening) does not take into account any information about the unigram probability. A back-off model will use discounted estimates of either the bigram distribution if the frequency of the event is above a given threshold, or the unigram probability if the event is infrequent. In both situations, only one of the distributions is directly used. An interpolated model makes use of both distributions by assigning $p_{\text {bg }}($ Monday evening $)=\alpha \tilde{p}($ evening $)+(1-\alpha) \tilde{p}($ evening $\mid$ Monday $)$ for some weight $\alpha$. While this incorporates both the unigram and bigram information, the resulting probability does not necessarily resemble what is observed in the data.

The same information can be included into a maximum entropy model by using the features:

$$
\begin{aligned}
& f_{1}(h, w)=\left\{\begin{array}{lll}
1 & : & \text { if } w=\text { evening } \\
0 & : & \text { otherwise }
\end{array}\right. \\
& f_{2}(h, w)=\left\{\begin{array}{lll}
1 & : \text { if } w=\text { evening and } h=\text { Monday } \\
0 & : & \text { otherwise }
\end{array}\right.
\end{aligned}
$$


where $h$ is the previous word. Unlike the bigram model, a maximum entropy model with constraints on features $f_{1}$ and $f_{2}$, on the other hand, does not require $p_{\text {me }}($ evening $)=\tilde{p}($ evening $)$ or $p_{\text {me }}($ evening $\mid$ Monday $)=\tilde{p}($ evening $\mid$ Monday $)$ in every case. Instead, it is only necessary that these features match their empirical targets on average over the training data. By placing restrictions only on feature expectations rather than directly on model probabilities, the maximum entropy model is able to simultaneously make use of the information provided by both features while at the same time remaining consistent with the observations.

Another useful property of features under the maximum entropy framework is that there is no need for them to be statistically independent. Multiple features may be used to provide constraints on the same target, and their interaction will be taken into account during the training process. This is particularly useful if features are to be derived from multiple, correlated knowledge sources. Part-of-speech information, for example, may be used to augment word-based information in language modelling. A feature such as:

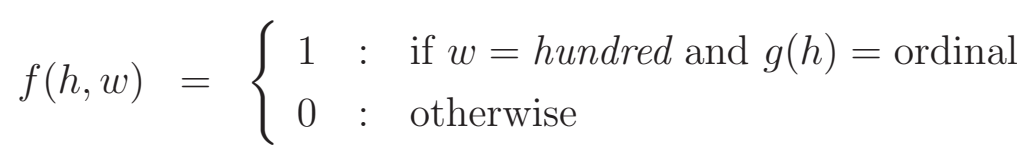

could be added to the set of standard $n$-gram features, where $g(h)$ gives the POS tag of the previous word. Importantly, adding information from a different knowledge source in this way does not require any changes to be made to the form of the model. Adding such information to an $n$-gram model, for example, may result in a fragmentation of the data into word-POS pairs, or require a decoupling of the variables by making assumptions of their independence, both of which are undesirable.

The flexibility of the maximum entropy model has meant it has successfully found use in a wide range of NLP problems. Berger et al. [139] described the use of maximum entropy models in statistical translation. A number of sub-tasks involved in the process, including context-sensitive word translation, segmentation and word reordering, were formulated under the maximum entropy framework. Rosenfeld [144] used a maximum entropy to incorporate trigger features in a language model, which significantly reduced perplexity. Ratnaparkhi [138] used maximum entropy models for variety of problems, including sentence boundary detection, POS tag assignment and parsing. He showed that state-of-the-art performance could be achieved on these tasks using features customised for the specific problem, but under the same modelling framework. 


\section{Chapter 5}

\section{Language Modelling with ToBI Features}

When training a language model for use in an ASR system, acoustic information is already available, and it may be worthwhile to incorporate this into the language model. The maximum entropy framework discussed in Chapter 4 is a theoretically sound method for combining multiple information sources, such as word and prosodic class sequences, in a statistical classifier. This only solves half of the problem, though, as it is still necessary to derive a set of constraints for the model. In order to be effective, these constraints must provide useful information to the classification task, and their target values need to be robustly estimated from a training sample.

This chapter will describe an approach for including prosodic information, in the form of ToBI features, into a language model. The first section will introduce a speech corpus that has been transcribed according to ToBI conventions. It will examine the distribution of these prosodic labels, and explain the simplified prosodic representation used in the experiments. The second section will describe some metrics for evaluating the potential contribution of the prosodic feature sets, and provide some supporting evidence for the use of combined prosodic and syntactic information. In the third section, the experimental setup will be detailed, and the form of the models and prosodic features explained. This section will also provide perplexity results for models, and offer some interpretations of their results. The fourth section of this chapter will present the results of speech recognition tests using the new language models. It will describe the baseline recogniser, as well as the multi-pass decoding process. 


\subsection{Corpus}

Training statistical acoustic and language models typically requires a large set of labelled speech data. While orthographically transcribed speech corpora are relatively common, few have any substantial amount of prosodic annotations. One of the largest corpora containing such prosodically transcribed speech is the Boston University Radio News Corpus (RNC) [145]. It consists of recordings of broadcast news stories, read by professional radio announcers.

The radio news speech style has some useful properties for prosodic analysis. A radio announcer must communicate a message through speech alone, and the use of prosody in this setting by may be directed more specifically towards aiding listener comprehension and reducing ambiguities. Professional speakers also use prosodic structure more consistently than non-professional speakers, as well as producing more clearly defined prosodic characteristics. While this speaking style is more controlled than that of spontaneous speech, the prosodic patterns of this type of speech can still be considered to be natural.

The corpus consists of approximately seven hours of speech, recorded from three female (F1A, F2B, F3A) and four male (M1B, M2B, M3B, M4B) speakers. It contains both recordings of original radio broadcasts as well as laboratory recorded simulations. A sampling rate of $16 \mathrm{kHz}$ was used for digitising the speech data. About 3 hours, or roughly half, of the speech has been annotated with phonetic alignments, orthographic transcriptions, part-of-speech tags and prosodic labels. Data from only one of the seven speakers, M4B, was not prosodically transcribed.

The POS tags of the Penn Treebank [146] are also provided in this corpus. The full set of tags consists of 22 open class categories, 14 closed class categories and 11 punctuation labels, for a total of 47 labels. The word categories of the Penn Treebank are listed in Appendix A. Tagging was performed automatically using BBN's probabilistic part-of-speech tagger [147], trained on sentences taken from the Wall Street Journal portion of the Penn Treebank. The tagger used a bigram model of tag sequences as well as lexical features. Analysis of the performance of this tagger using a subset of data which had manually corrected transcriptions found the error rate to be $2 \%$.

Prosodic transcription of the corpus was performed largely according to the ToBI conventions for American English [101], with a few minor extensions to the system. Seven types of pitch accents were used: $\mathrm{H}^{*}, ! \mathrm{H}^{*}, \mathrm{~L}+\mathrm{H}^{*}, \mathrm{~L}+! \mathrm{H}^{*}, \mathrm{~L}^{*}, \mathrm{~L}^{*}+\mathrm{H}$ and $\mathrm{H}+\mathrm{H}^{*}$. Similarly to the ToBI labels described in Section 3.3.2, L and H denote low and high tones, respectively, while !H represents a downstepped tone. The location 


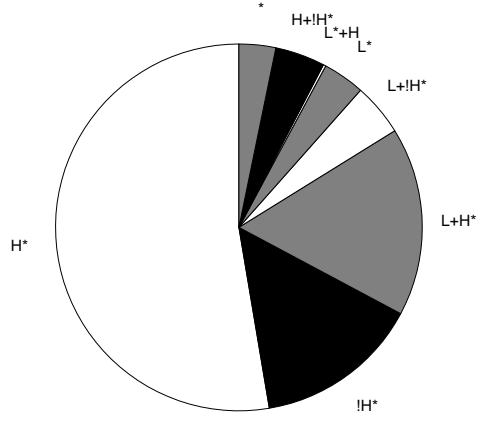

(a) Pitch accent distribution.

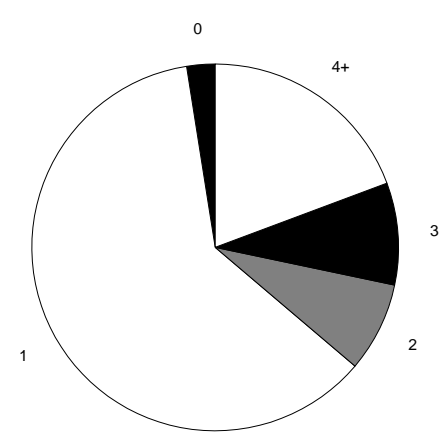

(b) Break index distribution.

FiguRE 5.1: Distribution of pitch accents and break indices in the training partition of the RNC data.

of the accent in bitonal events is indicated by the *. Intermediate phrase boundaries were marked in this corpus by L-, H- or !H-, and full intonational phrase boundaries were marked by either $\mathrm{H} \%$ or $\mathrm{L} \%$. Two additional break index values were also specified. The intonational phrase boundary, 4, was extended to additionally distinguish between groupings of intonational phrases, marked by a break index of 5 , as well as sentence boundaries corresponding to break index 6 .

Inter-transcriber agreement on the presence versus absence of pitch accents on the prosodically transcribed subset of this corpus was 91\% [145]. Among the words which were labelled as having accents, there was a $60 \%$ correspondence on pitch accent types, increasing to $80 \%$ when the $\mathrm{H}^{*}$ and $\mathrm{L}+\mathrm{H}^{*}$ accents were combined. For break indices, there was a $95 \%$ agreement when allowing for uncertainty by merging adjacent levels.

The distribution of pitch accents and break indices is shown in Figure 5.1. Break index values of 5 and 6 were not used in some transcriptions, so these were considered to be equivalent to 4. A number of categories, both in pitch accents and break indices, occur very infrequently. Lack of training data is a significant problem when training statistical models, and the rarity of these features may negatively impact on the model. Additionally, a small number of pitch accents (denoted by * or $\mathrm{X}^{*}$ ?) were marked as being accents, but their specific category was not indicated. For these reasons, we adopt an approach similar to other related work (e.g. [126]) and simplify both features to binary values. Words are then marked as being accented (a) or unaccented $(\mathrm{u})$, and the degree of prosodic juncture between two words may 
either be a regular word boundary (1, corresponding to break indices of $0-3$ in the standard notation) or a phrase boundary (4, corresponding to break indices of 4 or greater). The break index is therefore used to model the presence or absence of an intonational phrase boundary.

The data sets used in the following experiments were taken from the prosodically transcribed portion of this corpus. This consisted of approximately 3 hours of speech from six of the seven speakers (three male and three female), totalling about 30,000 words. The paragraph sized utterances were split into individual sentences, and randomly partitioned into three sets. $85 \%$ of the samples were used for training, $10 \%$ for testing, and the remaining $5 \%$ formed the development test set. This data organisation is similar to the multi-speaker speaker-dependent partitioning described in Hasegawa-Johnson et al. [148]. Log-likelihood gain on the development set was used to provide an early stopping criterion for the training algorithm.

\subsection{Evaluating Prosodic Features}

Chapter 3 described a number of potential ways in which prosody can assist speech recognition. In order to recognise words from continuous speech, correct segmentation is critical, and a number of prosodic attributes can act as cues to boundary locations. Prosodic phrasing, accentuation and deaccentuation are all related to syntactic structure. Two issues should be considered when applying this knowledge to an ASR system. Firstly, these prosodic events are difficult to quantify in terms of acoustic parameters. Sometimes, their correlation with acoustic features can be weak, or conditionally dependent on other factors. Secondly, the way in which a statistical model processes speech is fundamentally different to a human's. In general, the information which statistical models can apply is much more limited, and they are also significantly affected by noisy or unreliable training data.

The use of prosodic annotations which have been transcribed perceptually allows the first issue to be circumvented to a certain extent. While the development of a fully automatic system would require prosodic features to also be extracted automatically, ToBI transcriptions such as those provided by the RNC data offer a simple starting point for investigations. The second point is potentially more difficult to address. Computational limitations restrict the amount of information a model may use, as does inaccurate and sparse data. The choice of the maximum entropy model, though, does offer some advantages. In particular, there is no inherent restriction on the complexity of features other than computational expense. This is of particular use when modelling the relationship between prosodic and word sequences, as 
no assumptions about their independence need to be made. Since the interaction between prosody and words is quite complex, it is desirable to assume as little as possible about what has not been observed in data. This is a fundamental precept of the maximum entropy principle which is particularly applicable in this scenario.

Given a number of candidate features which may be included a model, it is often helpful to have an estimate of their potential contribution. This can allow for more informed choices regarding which features to incorporate, as well as provide some preliminary information on how they behave. One metric which can be used for this purpose is mutual information (MI). The MI, $I(X ; Y)$, between random variables, $X$ and $Y$, is a measure of the amount of information one of the random variables provides about the other. It is defined as:

$$
\begin{aligned}
I(X ; Y) & =H(X)-H(X \mid Y) \\
& =\sum_{x} \sum_{y} p(x, y) \log \frac{p(x, y)}{p(x) p(y)}
\end{aligned}
$$

MI is bounded below by zero, and above by $H(X)$. If $X$ and $Y$ are independent, then $p(x, y)=p(x) p(y)$, and $I(X ; Y)=0$. This is quite intuitive, as knowing $X$ does not provide any information about $Y$, and vice versa, if they are independent. Conversely, if $X$ and $Y$ are identical, then $H(X \mid Y)=0$, and $I(X ; Y)=H(X)=$ $H(Y)$. MI is also symmetric, so $I(X ; Y)=I(Y ; X)$. If $X$ carries some information about $Y$, then $Y$ also contains that information about $X$.

The effectiveness of a feature can be assessed by measuring the MI between the feature and the word it is being used to predict. A bigram feature, for example, uses the identity of the previous word as a predictor. The MI between a word, $w_{i}$, and the feature, $f\left(w_{i}\right)=w_{i-1}$, can then be approximated with respect to a training corpus:

$$
I(w ; f(w))=\frac{1}{N} \sum_{w_{i-1}, w_{i}} \log \frac{\tilde{p}\left(w_{i-1}, w_{i}\right)}{\tilde{p}\left(w_{i-1}\right) \tilde{p}\left(w_{i}\right)}
$$

where $N$ is the size of the training set and $\tilde{p}(\cdot)$ is the probability of the specified word, or word sequence, in the training data.

We have examined the mutual information between words and their prosodic labels. Due to the close relationship between prosody and syntax, we have also chosen to include syntactic information in the analysis. POS tags are used as the basic units bearing syntactic information, as these are already provided in the corpus. It is worth noting, though, that POS labels are a fairly weak representation of syntax. Full, or even partial parse trees would be capable of describing syntactic 
properties more thoroughly. Explicit markers of syntactic phrases, for example, could be expected to show strong correlations with prosodic phrasing. On the other hand, parse trees are substantially more difficult to estimate automatically. In the absense of a reliable source of detailed syntactic information, POS labels are used as an alternative, since automatic tagging of unlabeleld text can be performed quickly and accurately. The prosodic and syntactic context of a word, $w_{i}$, is represented by the following information:

- $a_{i}$ - The pitch accent label associated with $w_{i}$ (which may be either ' $\mathrm{u}$ ' or 'a').

- $b_{i}$ - The break index label occurring at the boundary between words $w_{i}$ and $w_{i+1}$ ('1 or ' 4 ').

- $b_{i-1}$ - The break index label occurring at the boundary between words $w_{i-1}$ and $w_{i}$ (' 1 ' or ' 4 ')

- $s_{i}$ - The POS label associated with word $w_{i}$ (one of the Penn Treebank tags).

As described in the previous section, each of the prosodic labels can take one of two values. This simplification means that the presence of intonation phrase boundaries $(\mathrm{H} \%, \mathrm{~L} \%)$ is equivalent to a break index of 4 . A word $w_{i}$ is phrase-initial if it immediately follows a boundary, and this is indicated by $b_{i-1}=4$. Similarly, a word is phrase-final if it occurs immediately before a word boundary $\left(b_{i}=4\right)$. Because only two classes are used, the entropies of these features are small and subsequently, MI between these features and the predicted word will also be small. Used on their own, they would make for very poor predictors. However, the purpose of including this prosodic information is not to replace the $n$-gram features, but rather to supplement them. If the information they contain is not redundant with that provided by the $n$-grams, then they can still contribute useful knowledge to the prediction. To measure how much excess information is provided by one variable given that some information is already known, MI can again be used, by conditioning it on the known quantity. The conditional mutual information between $X$ and $Y$, given $Z$ is known, is defined as:

$$
\begin{aligned}
I(X ; Y \mid Z) & =H(X \mid Z)-H(X \mid Y, Z) \\
& =\sum_{x, y, z} p(x, y, z) \log \frac{p(x, y \mid z)}{p(x \mid z) p(y \mid z)}
\end{aligned}
$$

Similarly to MI, this can be approximated by a summation over the training data. Table 5.1 shows these measures for the three features, individually and in various 


\begin{tabular}{cccc}
\hline$f\left(w_{i}\right)$ & $H(f)$ & $I\left(w_{i} ; f\left(w_{i}\right)\right)$ & $I\left(w_{i} ; f\left(w_{i}\right) \mid w_{i-1}\right)$ \\
\hline \hline$a_{i}$ & 1.02 & 0.62 & 0.55 \\
$b_{i}$ & 0.69 & 0.41 & 0.39 \\
$b_{i-1}$ & 0.69 & 0.33 & 0.23 \\
$s_{i-1}$ & 3.96 & 1.99 & 0.36 \\
$\left(a_{i}, s_{i-1}\right)$ & 4.92 & 2.70 & 0.86 \\
$\left(b_{i}, s_{i-1}\right)$ & 4.62 & 2.51 & 0.71 \\
\hline
\end{tabular}

TABLE 5.1: Mutual information between a word and its features over the training set. The last column shows mutual information conditioned on a bigram context.

combinations. The first column is the entropy of the feature. The second column contains the MI between the feature and the third column is the MI conditioned on the previous word.

Unsurprisingly, the absolute values for $I\left(w_{i}, f\left(w_{i}\right)\right)$ are low for both types of prosodic features. However, conditioning on the previous word results in minimal decrease in MI. This contrasts with the POS information which, while having a much higher entropy, experiences a drastic drop in MI when conditioned on the previous word. The information provided by the prosodic features, on the other hand, is largely unaccounted for by the bigram features, so gains might be achieved by using them in addition to the bigrams. These results also indicate that a word shares significantly more information with the break index label occurring at the end of that word than the break at the beginning. This is also the case for conditional MI. One factor contributing to this is the difference in types of words occurring on either side of the boundary. Closed class words are much more likely to appear in phraseinitial positions than phrase-final positions. There are only a small number of words in the closed class set, so fewer unique words are observed immediately following a phrase boundary. In the training data, 1827 different words occurred before a phrase boundary, while only 637 different words occurred after the boundaries. As a result, there is less information is contained in phrase-initial words: these have 7.25 bits of entropy, compared with 10.25 bits for phrase-final words. Therefore, mutual information is reduced accordingly.

Another interesting result is seen for the case of the combined prosodic-syntactic labels, $\left(a_{i}, s_{i-1}\right)$ and $\left(b_{i}, s_{i-1}\right)$. Looking down the column of MI values, we can see that, $I\left(w_{i} ;\left(a_{i}, s_{i-1}\right)\right)>I\left(w_{i} ; a_{i}\right)+I\left(w_{i} ; s_{i-1}\right)$, and similarly, $I\left(w_{i} ;\left(b_{i}, s_{i-1}\right)\right)>$ $I\left(w_{i} ; b_{i}\right)+I\left(w_{i} ; s_{i-1}\right)$. The MI between $w_{i}$ and the combined label is greater than the sum of MI values of each individual component. This extends to the label combining all three types of information, $\left(a_{i}, b_{i}, s_{i-1}\right)$. The increase in MI indicates 
that the presence of the syntactic labels increases the correlation between a word and its prosodic label. This positive interaction information provides motivation for combining syntactic and prosodic knowledge from an information theory perspective, as there is some synergy between these sources.

While the MI values of the prosodic features may indicate that they reduce the uncertainty of the predicted word, it does not guarantee that modelling those features will improve classification performance. MI shows the amount of information which is shared between the variables, and represents an upper bound on the potential gain. It does not consider how the features interact when they are combined in a model. Also, in the case of these prosodic features which have relatively low MI compared to $n$-gram features, the additional knowledge they provide may be inconsequential. If the model can predict a word accurately using only $n$-grams, then any extra reduction in uncertainty will not affect the outcome. Conversely, if the language model considers a correct word sequence to be sufficiently unlikely, then a relatively minor boost to its probability from the inclusion of prosodic information may still have no impact on the final classification.

\subsection{Language Modelling}

\subsubsection{Baseline}

The baseline language model used for comparison was a standard trigram model. It used modified Kneser-Ney smoothing, as this exhibited superior performance to the other smoothing techniques in an evaluation using our data. Free parameters were calculated according to the heuristics shown in Equation (2.7). This model was constructed using the SRI Language Modeling (SRILM) toolkit [149]. Although this model is used as a baseline to compare against the prosodically augmented maximum entropy models, it should be noted that silences are explicitly modelled as part of the $n$-gram sequences. Since pausing can be considered to be a prosodic feature, the trigram is therefore not completely independent of prosody.

\subsubsection{ToBI features}

We formulated some simple features for directly incorporating the prosodic and syntactic contextual information. These are described in terms of a class label $w$ and the context, $h$. In all cases, $w$ is a member of the set of words in the training vocabulary, and the context consists of the sequence $h=\left(h_{1}, \ldots, h_{i}\right)$. Each element in $h$ represents word, pitch accent, break index and POS information as 
a 4 -tuple $(w, a, b, s)$, with the exception of the last term, $h_{i}$. Because $w_{i}$ is the word to be predicted, and $s_{i}$ is highly dependent on $w_{i}$, these are excluded from the context. Therefore, $h_{i}$ comprises prosodic information only, giving the full context as $h=\left(\left(w_{1}, a_{1}, b_{1}, s_{1}\right), \ldots,\left(w_{i-1}, a_{i-1}, b_{i-1}, s_{i-1}\right),\left(a_{i}, b_{i}\right)\right)$.

From a linguistic perspective, $n$-grams fail to capture much of the important information about word sequences. In practice, though, they are powerful predictors for statistical models, and the sophisticated smoothing algorithms available to them mean $n$-grams are well suited to dealing with the sparse nature of natural languages. Therefore, it is essential that this information is also used in a prosodic model. Under the maximum entropy framework, $n$-gram information can be included by encoding them as the following feature functions:

$$
f_{\mathrm{ng}}(h, w)=\left\{\begin{array}{lll}
1 & : & \text { if } w=w_{i} \text { and } w_{i-n+1}^{i-1} \in h \\
0 & : & \text { otherwise }
\end{array}\right.
$$

for $n=1,2,3$, corresponding to unigrams, bigrams, and trigrams respectively. For convenience we use the notation $x \in h$ to denote that the appropriate subset of $h$ is equal to some label, or sequence of labels, $x$. For example, the bigram feature $f_{\text {the group }}(h, w)$ would be active if $w_{i}=$ group, and $h_{i-1}=($ the $, u, 1, D T)$. It would also be active for any other combination of pitch accent, break index and POS label in $h_{i-1}$. This latter condition is indicated as the $\in h$.

To incorporate the prosodic information, $n$-gram-like features were derived for pitch accents, $f_{a}(h, w)$, break indices, $f_{b}(h, w)$ and POS tags $f_{s}(h, w)$ :

$$
\begin{aligned}
& f_{a}(h, w)= \begin{cases}1 \quad: & \text { if } w=w_{i} \text { and } a_{i-m+1}^{i} \in h \\
0 & : \text { otherwise }\end{cases} \\
& f_{b}(h, w)= \begin{cases}1 \quad: & \text { if } w=w_{i} \text { and } b_{i-m+1}^{i} \in h \\
0 & : \text { otherwise }\end{cases} \\
& f_{s}(h, w)= \begin{cases}1 \quad: & \text { if } w=w_{i} \text { and } s_{i-m+1}^{i-1} \in h \\
0 \quad: & \text { otherwise }\end{cases}
\end{aligned}
$$

These features are active if a given sequence of labels (e.g. $\left.\left(a_{i-m+1}, \ldots, a_{i}\right)\right)$ is present in the most recent $m$ length context, where $m>0$. The break index features were only used to model the presence of a boundary after a given word, as a word's phrase-initial status can be captured by increasing the length of the feature.

By modelling prosodic context separately from the word context, the increase in parameter count of the prosodic models is kept minimal. This approach is valid 
because the model does not require there to be any independence between features. While a large amount of redundancy is not desirable, a feature selection procedure can be applied to prune off those features which do not contribute any additional information to the model. Another benefit of not jointly modelling prosodic and word contexts is that it can allow greater history lengths to be used. Sparse data is the primary reason $n$-gram models are restricted to using a very short context. The small number of possible prosodic classes, on the other hand, means the expectations of prosodic feature can be estimated robustly from empirical data over much longer distances. All pitch accent sequences of length 4, for example, were observed a minimum of 300 times in the training data. Therefore, we extended the lengths of these prosodic and syntactic features beyond the history size of two which was used for standard $n$-grams.

\subsubsection{Perplexity results}

As a proof of concept, we trained some maximum entropy models using the features described in Section 5.3.2, and evaluated their perplexities. Because we used the manually transcribed labels during the testing process, these values can be considered to be what is attainable in the best case scenario. While this is not practicable, it gives an indication of how much information is contained in the prosodic features, and how well the models can extract that information and apply it to the task. Inaccuracies in automatic labelling methods, which will be discussed in greater detail in the next chapter, would reduce the amount of useful information available, although it should be remembered that even the manual transcriptions are not necessarily completely accurate. In our experiments, ToBI labels were paired up with words based on time. A word was labelled as accented if an appropriate pitch accent occurred within its duration. Break index labels were distributed to the nearest word edge.

All features in the models were constrained to equal their empirical values in the training set. Smoothing and feature selection were handled by applying an exponential prior over model parameters, with the smoothing parameters chosen according to Equation (4.13). The maximum entropy classification library of Kazama and Tsujii [141] was used, which in turn used Benson and Moore's [150] limited memory variable metric bounded constrained optimisation routine for parameter estimation. Training was performed either until convergence or until the likelihood on the heldout set stopped increasing. Perplexities were quite sensitive to the variance of the prior, and this value was optimised separately for each model using the development 


\begin{tabular}{ccccc}
\hline model & $\mathrm{m}=1$ & $\mathrm{~m}=2$ & $\mathrm{~m}=3$ & $\mathrm{~m}=4$ \\
\hline \hline LM-A & 82.77 & $\mathbf{7 8 . 7 8}$ & 80.61 & 85.64 \\
LM-B & 78.60 & 73.38 & $\mathbf{7 2 . 9 3}$ & 73.72 \\
LM-S & - & 76.10 & $\mathbf{7 1 . 5 4}$ & 72.09 \\
LM-ABS & - & 71.47 & $\mathbf{7 0 . 4 2}$ & 71.22 \\
\hline
\end{tabular}

TABLE 5.2: Perplexities of language models using features comprising length $m$ sequences of ToBI labels.

test set.1 Test set perplexities of the maximum entropy models are shown in Table 5.2, for various values of $m$. Models LM-A, LM-B and LM-S are the models using trigram features and one of either pitch accent, break index or POS features, as described by Equation (5.2), Equation (5.3), and Equation (5.4), respectively. The previous MI results indicated that there may be benefits to combining these features, so another model, LM-ABS, was trained to use all three types of features.

The perplexity of the baseline model was 86.46. When pitch accent features are included, perplexity drops to a minimum of 78.38 at $m=2$, representing a relative reduction of $9.3 \%$. The lowest perplexity achieved by model B, at $m=2$, was 72.93 , or a $15.6 \%$ reduction. This is significantly better than the pitch accent model, which is in contrast to what the mutual information results suggest. Comparing the probabilities the two models assign to the same word sequences shows that model $\mathrm{B}$ consistently gives higher scores when a boundary appears in the context. This is supported by an examination of the model parameters corresponding to the break index features, which are almost always positive. While there are fewer different break index features than pitch accent features overall, the break index features, on average, were observed more consistently with respect to the predicted words. This allows the model to assign greater relative weights to these features, and hence their presence in the context increases probabilities correspondingly. Essentially, the smaller space of break index and word pairs leads to a significant perplexity reduction, and this factor is not taken into account by the MI test.

Incorporating syntactic information also leads to significant gains. Using these features alone, the model achieved a perplexity of 71.54. Moreover, the POS features represent a sufficiently different type of information to the two prosodic features that using them in conjunction further improves perplexity over any of the individual feature sets. Combining all three types of features, in LM-ABS, resulted in the best performing model, having a perplexity 70.42 , which is $18.6 \%$ lower than the baseline. This also demonstrates that the maximum entropy model is able to make use of

\footnotetext{
${ }^{1}$ The actual values of $W$ were between 0.1 and 0.3 .
} 
additional knowledge sources without degrading the effectiveness of the original information.

While the inclusion of pitch accent, break index and POS information all improved perplexity, the effectiveness of modelling longer sequences of these features was limited. The optimal length for the sequences of pitch accents was $m=2$, which is equivalent to the history length used by bigram features. While perplexity decreased further at $m=3$ for both POS and break index features, the improvement for the break index features was only very slight. As the lengths of the prosodic and POS features were increased beyond these small values, perplexities rose. Although data sparsity is less of an issue with the small number of prosodic and POS classes, there are other factors which may cause this. It may simply be the case that the contextual information beyond this length does not contain useful information. The average amount of shared information contained in word pairs decreases quickly as the distance between them increases [144], and it is not unreasonable to expect that a similar reduction would also be experienced by other knowledge sources. Given the much lower initial information content of the prosodic and syntactic features, their contribution at longer distances would quickly become negligible. It should be noted, however, that while these results indicate that no gains are obtained for these longer span features, it does not mean that long distance prosodic and syntactic contexts are not useful. Rather, it is only the information represented by these particular sequences of pitch accent, break index and POS labels which does not assist the classification.

Another possible explanation for ineffectiveness of the longer distance prosodic features may be that the prosodic labelling is not consistent enough to use as a predictor. Differences in the ways the various speakers used prosodic markers could also be a contributing factor to this. Ostendorf et al. [145] noted that, in the RNC data, speaker F2B used phrase boundaries, pitch accents and low phrase tones more frequently than speaker M1B. Reducing the number of values each variable may take reduces the degree of inconsistency, but differences can still be seen in the distribution of accent and boundary usage in our training data, as shown in Table 5.3. While a small level of unreliability in the transcriptions does not severely hinder the model's ability to effectively use short sequences, attempting to model longer sequences of these labels would exacerbate the inconsistencies and subsequently lead to a degradation in classification performance. 


\begin{tabular}{lllllll}
\hline & F1A & F2B & F3A & M1B & M2B & M3B \\
\hline \hline a & $44 \%$ & $43 \%$ & $44 \%$ & $41 \%$ & $46 \%$ & $44 \%$ \\
4 & $22 \%$ & $27 \%$ & $19 \%$ & $19 \%$ & $23 \%$ & $18 \%$ \\
\hline
\end{tabular}

TABLE 5.3: Distribution of pitch accents and break indices in the training set. Speaker M2B used pitch accents more frequently than the other speakers, while speaker M1B used fewer. Speaker F2B used substantially more phrase boundaries than the other speakers.

\subsection{Speech Recognition}

Having achieved reasonable perplexity reductions, we proceeded to perform speech recognition experiments using these language models in conjunction with an HMMbased recogniser built using the Hidden Markov Model Toolkit [151]. The raw speech data were parameterised into sequences of 39 dimension vectors containing 12 MFCCs plus the $0^{\text {th }}$ coefficient, and their first and second order derivatives. Feature vectors were calculated using a frame period of $10 \mathrm{~ms}$. These were modelled by 5 mixture Gaussians and used as observations of context-dependent triphone models. Models had 3 states and no skips. The recogniser was trained and tested using the same data sets as the language models.

For these speech recognition experiments, we also include results for two additional models which model the distributions of word-prosody tuples, $(w, p)$, where $p$ is the combined accent and break index label $(a, b)$. Both models are based on those described by Chen and Hasegawa-Johnson [126]. The first, LPDM, calculates probabilties directly using $n$-gram estimates:

$$
p\left(w_{i}, p_{i} \mid w_{i-2}^{i-1}, p_{i-2}^{i-1}\right)=\frac{C\left(w_{i-2}^{i}, p_{i-2}^{i}\right)}{C\left(w_{i-2}^{i-1}, p_{i-2}^{i-1}\right)}
$$

where $C(\cdot)$ is the count of the specified event in the training data. The second model, LPDC1, attempts to obtain a more robust estimation by conditioning probabilities on POS labels: 2

$$
p\left(w_{i}, p_{i} \mid w_{i-2}^{i-1}, p_{i-2}^{i-1}\right)=\sum_{s_{i-2, i-1, i}} p\left(p_{i} \mid s_{i-2}^{i}, p_{i-2}^{i-1}\right) p\left(s_{i-2}^{i} \mid w_{i-2}^{i}\right) p\left(w_{i} \mid w_{i-2}^{i-1}, p_{i-2}^{i-1}\right)
$$

These two models operate on hypotheses containing both words and prosodic labels. For evaluation, we disregard the prosodic labels when comparing them to the maximum entropy models. That is, we consider all prosodic variants of a hypothesised

\footnotetext{
${ }^{2}$ This is the second order extension of Equation (5) in [126].
} 
word to be correct so long as the word component is correct.

\subsubsection{Multi-pass decoding}

Training and evaluating maximum entropy models is computationally expensive, so it is common to apply them to a reduced search space in the later stages of a muti-pass decoding approach. In these experiments, an $N$-best list was obtained from the standard speech recogniser, which was re-ranked using the new language models. We chose to rescore $N$-best lists rather than word lattices for a number of reasons. Firstly, $\mathrm{N}$-best list rescoring is simple to perform, and relatively fast for reasonable sized lists. Rescoring lattices can be much slower. Secondly, maximum entropy models allow for the use of information arbitrarily far back in the context. This violates the dynamic programming assumption used by the Viterbi decoding algorithm. A consequence, then, is that the decoder may prefer paths to which the original $n$-gram model assigns the highest probability, while overlooking other paths which initially have lower scores. $A^{*}$ decoding can be used as an alternative, but the problem then shifts to obtaining a suitable estimate of the remaining probabilities of partial paths. Och et al. [152] used the function:

$$
p\left(w_{i}\right)=\max _{\left(w_{i-2}, w_{i-1}\right)} p\left(w_{i} \mid w_{i-2}, w_{i-1}\right)
$$

as an upper bound on the probability of each word. This is an admissible heuristic and will guarantee an optimal solution for a trigram language model. However, it is not directly applicable to a maximum entropy model in general. Evaluating over all possible histories is feasible for the trigram, but an event in the maximum entropy model may have arbitrarily many features associated with it, and these features may be dependent on very large histories. An approximation to this value can be obtained by selecting the maximum value over the training set; however, it is then no longer guaranteed to be admissible. In [152], this function was combined with a number of other estimates to derive the final heuristic. We have found that, on its own, this is too loose a bound, and results in prohibitively long decoding times.

Nguyen et al. [153] evaluated an $N$-best rescoring approach against lattice decoding, and found little difference in word accuracies. In some cases, rescoring the large number of hypotheses available in the lattice resulted in a performance degradation, as the higher level knowledge sources selected an incorrect hypothesis which was not present in the $N$-best list. They also note that $N$-best lists provide advantages for experimentation, as it eliminates the need to develop a general search strategy, as well as allowing for more rapid optimisation of tunable parameters. 


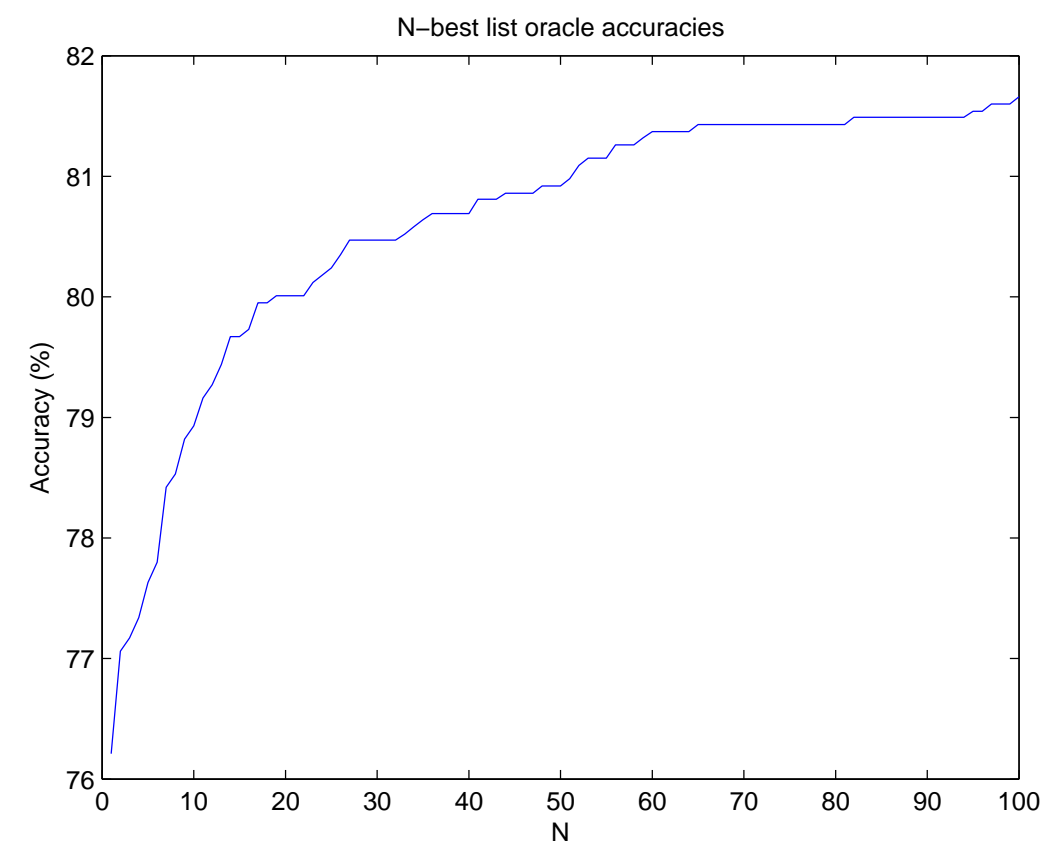

FiguRE 5.2: Oracle accuracies of $N$-best lists for $1 \leq N \leq 100$.

In order to select an appropriate size for the $N$-best list, we examined the oracle accuracies for up lists of up to 100 hypotheses per utterance. The oracle accuracy is the accuracy of the hypothesis in the list which is closest to the reference transcription. It is therefore the upper bound on the possible improvement which can be achieved by rescoring a list of that size. The oracle accuracies are shown in Figure 5.2. At $N=1$, the word accuracy is $76.21 \%$. This is also the accuracy of the trigram baseline. Accuracies increase fairly steeply as $N$ increases for small values of $N$, and plateaus at around $N=65$. At this point, the accuracy is $81.43 \%$. From $N=65$ to $N=100$, it only rises a further $0.23 \%$, to $81.66 \%$. Based on these results, we have chosen to rescore 65 -best lists in the following experiments. The grammar scaling factor was held fixed at 15.0 for all models, and no word insertion penalty was used.

\subsubsection{Results}

The word error rates for the language models are presented in Table 5.4. Both the inclusion of pitch accent features and break index features improved recognition accuracy, to $76.94 \%$ and $77.12 \%$, respectively. These gains over the $76.21 \%$ baseline were significant at the level of $p<0.05$, according to the MAPSSWE test described in Section 2.4.2. The improvements in recognition rate, and the effect of increased feature lengths, are also fairly consistent with the perplexity results. Combining the 


\begin{tabular}{ccccc}
\hline model & $\mathrm{m}=1$ & $\mathrm{~m}=2$ & $\mathrm{~m}=3$ & $\mathrm{~m}=4$ \\
\hline \hline LM-A & 76.77 & $\mathbf{7 6 . 9 4}$ & 76.55 & 76.77 \\
LM-B & 76.83 & 76.89 & $\mathbf{7 7 . 1 2}$ & 76.89 \\
LM-S & - & 76.89 & $\mathbf{7 7 . 0 6}$ & 77.00 \\
LM-ABS & - & $\mathbf{7 7 . 5 1}$ & 77.40 & 77.12 \\
LPDM & - & - & 75.86 & - \\
LPDC1 & - & - & 77.00 & - \\
\hline
\end{tabular}

TABLE 5.4: Recognition accuracies of language models using ToBI features.

three sets of features gives the best model with an accuracy of $77.51 \%$. This $1.3 \%$ absolute gain is significant at the level of $p<0.01$.

The model LPDC1 also improved on the baseline, but the gain of $0.8 \%$ was less than that reported by Chen and Hasegawa-Johnson [126]. While the $1.2 \%$ gain of LPDC1 over LPDM is comparable, the model LPDM itself performed worse than the word-only trigram. In contrast to the $0.35 \%$ performance loss seen here, the bigram version of LPDM evaluated by Chen and Hasegawa-Johnson improved upon the word-only bigram by $1.4 \%$. We believe the reason for this discrepancy is that the approach of introducing each prosodic variant of a word as a distinct entity in the vocabulary scales poorly with context length. Therefore, while the assumptions and approximations made by this method are valid, the trigram variants of the models LPDM and LPDC1 suffer considerably more from data sparsity problems than their bigram counterparts, and their performance degrades accordingly.

A more detailed breakdown of the results for the best performing variants of LM-A and LM-B is presented in Table 5.5. It is evident that the primary source of the improvements of these two models is the reduced number of substitution errors. All three models had a comparable number of insertion errors, while the two prosodic models showed a very slight reduction in deletion errors. The corrected deletions almost always occurred near a corrected substitution, so the lower number of deletion errors is more likely a side-effect of corrected substitutions rather than an ability of the prosodic features to repair this type of error.

While the improvements to recognition accuracy are the result of an interaction between multiple features, it is still possible to identify some cases in which specific prosodic features may have contributed significantly to the correction. Consider the following partial transcriptions from the trigram and LM-A, together with the alignment of pitch accents: 


$\begin{array}{cccccccccc}\text { accent: } & \ldots & \mathrm{u} & \mathrm{a} & \mathrm{u} & \mathrm{u} & \mathrm{a} & \mathrm{a} & \mathrm{a} & \ldots \\ \text { trigram: } & \ldots & \text { as } & \text { well } & \text { as } & \text { four } & \text { another } & \text { revenue } & \text { raising } & \ldots \\ \text { LM-A: } & \ldots & \text { as } & \text { well } & \text { as } & \text { for } & \text { another } & \text { revenue } & \text { raising } & \ldots\end{array}$

In this scenario, no pitch accent is observed in the vicinity of the hypothesised words for/four. The word four, though, is much more likely to be accented than for. In the training data, an accented four was observed 26 times, while its unaccented form occurred only 5 times. In contrast, the word for was accented only 6 times in the training set, and it was unaccented the other 257 times. Subsequently, when the word four is encountered with no pitch accent, the model considers this to be an unlikely event and decreases the score of the associated hypothesis. Observing the unaccented form of for, on the other hand, is well supported by the training data, and hence its probability is increased relative to the alternatives. The increased probability of the latter hypothesis, in this case, was sufficient to raise its rank above the original and eliminate the substitution error.

Similar situations can be identified for the break index model. Consider the following transcriptions:

$\begin{array}{cccccc}\text { break: } & \ldots & 1 & 1 & 4 & 4 \\ \text { trigram: } & \ldots & \text { at } & \text { the } & \text { was } & \text { university } \\ \text { LM-B: } & \cdots & \text { at } & \text { the } & & \text { university }\end{array}$

The hypothesis which received the highest trigram probability is dispreferred by the maximum entropy model due to the co-occurrence of the word was with a boundary. Because the model is capable of identifying the fact that was is highly unlikely to be a phrase-final word, it considers the second option to be the better alternative. The occurrence of two consecutive boundaries is also somewhat unusual, so this further contributes to the decrease in the score of the original hypothesis.

In general, the improvements made by both LM-A and LM-B were the result of downgrading the rank of hypotheses which conflicted with the prosodic information. Of course, the opposite effect can also occur. If an event which is prosodically uncommon does occur, such as a phrase-final function word, the new model may incorrectly prefer other alternatives. The final score, though, is determined by the combination of active features for the relevant events, and on average, the corrections made by the addition of prosodic features outweigh any new errors they introduce.

In both of the previous examples, the original errors involved the misrecognition of a closed-class word. These types of errors form a significant proportion of overall recognition errors. The fact that corrections to such errors are made by the 


\begin{tabular}{cccc}
\hline Model & Deletions & Substitutions & Insertions \\
\hline \hline baseline & 56 & 308 & 55 \\
LM-A & 53 & 297 & 56 \\
LM-B & 53 & 294 & 56 \\
\hline
\end{tabular}

TABLE 5.5: Misrecognitions of three systems according to error type.

prosodic features supports the notion that the recognition of closed-class words is more dependent on prosody than open-class words [74]. However, in the two examples above, knowledge of the prosodic properties associated with the closed-class word alone is not sufficient to perform the correction. Rather, it is the disparity between the prosodic contexts of open and closed-class words which allows the model to successfully eliminate some of these errors.

The combined model, LM-ABS, outperformed the models using only a single set of features, but the additional gain over each individual model was not very large. This can be explained by the fact that the different features could, and often did, lead to the same correction. This is to be expected, as the phenomena that are modelled by the pitch accent and break index features are not independent. Of the corrections made by LM-A, over half were also shared by LM-B. The combination of features is still useful though, as they could prevent errors which were introduced by a single set of features. This is reflected in both the higher recognition rate of the combined model, as well as its improved statistical significance.

While the matched pairs test indicated the gains of the maximum entropy model were statistically significant, the gains themselves were quite small. A likely explanation for this is that the prosodic features, given that they only provide supplementary information to the core $n$-gram information, have a very small influence on the model. Therefore, the scores assigned by the prosodic model only deviate slightly from the baseline. When combined with the acoustic model scores, the contribution of the prosodic features is diminished even further. An increase in recognition accuracy under these circumstances, then, can only be achieved if hypotheses superior to the first hypothesis in the $N$-best list occur in high ranking positions. To examine if this is the case, we track the position of the oracle hypothesis in both the original $N$-best list and the list after re-ranking by LM-ABS, for the utterances in which it was not already ranked first. A histogram of the data is shown in Figure 5.3.

From this plot, it can be seen that the maximum entropy model does in fact have a significant effect on the ordering of the $N$-best list. In particular, there is a distinct shift of the oracle transcription into the top 5 hypotheses. Further 


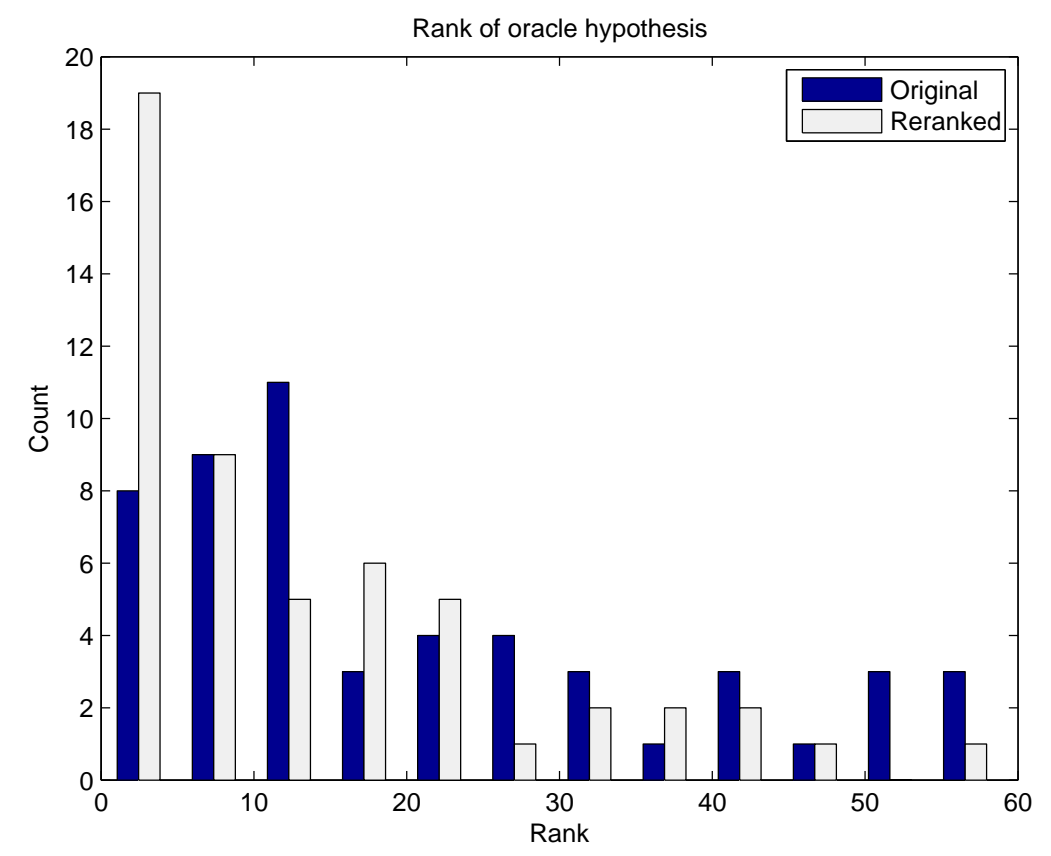

FiguRE 5.3: Histogram of the rank of the oracle hypothesis before and after reranking by LM-ABS. Each pair of bars represents a bin of 5 hypotheses.

evidence of this shift is seen in the drop in average rank of the oracle hypothesis, from 20.4 in the original list, to 13.8 after re-ranking. While the new language model significantly improves the rank of the best transcription, placing it in the top 5 hypotheses 19 times, the hypothesis was ranked outright first in only 6 of these cases. The remaining times, other hypotheses existed for which the model assigned an even higher score. This behaviour can possibly be attributed to the features' lack of discriminatory power. It is reasonable to expect that, of the 65 hypotheses present in the $N$-best list, more than one will be consistent with the prosodic context. Beyond the closed-class/open-class word distinction, the prosodic information is limited in its ability to distinguish between words. A misrecognition such as the substitution of tape for cape, then, is unlikely to be corrected by prosodic, or even syntactic information. These features merely serve to elevate the ranking of a subset of hypotheses. If, within this subset, there remain incorrect hypotheses, one of these may still be selected in preference to the oracle. 


\subsection{Conclusion}

In this chapter, we have formulated some simple prosodic features for use in a maximum entropy language model. These features were derived from a perceptually transcribed corpus according to the ToBI conventions. The reduced form of these transcriptions, into a binary opposition of pitch accents and break indices, showed a synergy with syntactic information in the form of POS tags in an examination of the mutual information between words, prosody and syntax. Combining this information into the language model led to a $19 \%$ decrease in perplexity over the baseline trigram model, and an absolute gain of $1.3 \%$ in word accuracy.

The results of the recognition tests correlated strongly with the perplexity results, and both experiments indicated that the most useful prosodic information was contained in the local context of the word. Extending the modelled context beyond one or two words, though, led to a degradation in both perplexity and word recognition rates. This, we believe, was due to the reliability of the labelling being insufficient for the purposes of training a statistical model.

Through an examination of the re-ranked $N$-best list, we have found substantial improvements to the rank of the oracle transcription in the list, which indicates a potential for further gains. It is possible that more detailed modelling of prosody, beyond the simple binary opposition of pitch accents and break indices, may help to discriminate between multiple hypotheses which are all consistent with the simplified representation of prosodic context. Using the full set of ToBI labels is one way in which more detailed prosody modelling may be achieved. Our analyses in this chapter, though, have not supported this approach. An alternative is to use the acoustic-prosodic features, such as F0 and energy, directly. This will be examined further in the next chapter. 


\section{Chapter 6}

\section{Automatic Feature Extraction}

Perceptually annotating speech, even when using a well-defined framework such as ToBI, is a labour-intensive process and requires trained labellers to achieve high inter-transcriber consistency. A fundamental requirement for training statistical models is the need for sufficient data to accurately represent the true distribution of the modelled classes, and it is difficult to meet this requirement when the data generation requires significant human effort. This is reflected in the very limited numbers of prosodically transcribed speech corpora in comparison to other corpora. Given the relative abundance of raw speech data, it would be desirable to be able to extract prosodic information from the speech in an unsupervised fashion.

This chapter will describe processes by which acoustic-prosodic features can be extracted automatically from a speech signal. The first section will provide a general overview of the main aims of automatic feature extraction in the context of this work, and how these features may be used. The second section will describe how the acoustic correlates of prosody are extracted from the signal. It will detail normalisation methods for these data, as this is an important step when dealing with the highly speaker-dependent prosodic information. We focus on extracting this information at the word level, and introduce a set of features tailored to this purpose. In the third section, an analysis of a number of features derived from the automatically extracted data is performed. The focus of this analysis is to determine how useful the features are for identifying prosodic events at the word level. A summary of how these features relate to the various prosodic events is given in the final section. 


\subsection{Aims}

There is a substantial amount of research exploring the problem of labelling prosodic phenomena using cues extracted directly from a speech signal (e.g. [98,100]). Often, this focuses on features which are correlated with prosodic events such as boundaries, accentuation and stress markers. While language modelling is a different task to recognising prosodic events, this information is not entirely irrelevant. We have shown in Chapter 5 that accentuation and prosodic phrase boundaries carry information which is helpful to word prediction. If these prosodic events can be automatically detected and classified, then it allows language models such as those previously described to be developed. These features can also be used directly in the model if desired, and this will be explored further in the next chapter.

In this work, we are primarily concerned with discerning whether a particular word is associated with an accent or boundary. We assume that a (possibly incorrect) transcription is available, from which we can obtain word identities and segmentation information. Labelling words with prosodic events in this scenario is a somewhat simpler task than attempting to identify prosodic events from the speech signal alone, as the available lexical information provides a valuable source of knowledge. For speech synthesis systems such as Festival [154], prosody prediction is performed solely using this lexical information.

Previous studies of this nature, such as the work of Milone and Rubio [155], have focused on the relationship between acoustic parameters and individual syllables, rather than words. While syllables are the basic unit with which accents are associated, we have assumed that only word level information is accessible. We therefore wish to examine how well prosodic events correlate with the raw acoustic parameters extracted over word regions.

\subsection{Prosodic Feature Extraction}

Duration, fundamental frequency, and energy are considered to be the primary acoustic correlates of prosody. This is well supported by experimental evidence, although findings with regard to the relative importance of each have varied [63]. Differences between languages, dialects, speakers and speaking styles may all be contributing factors to the variations in results. In this section, we examine the relationship between various acoustic features and prosodic events in the RNC data. As with the previous experiments, we focus on differentiating only between the presence and absence of pitch accents and phrase boundaries. Because we would eventually 
like to use these features in conjunction with a language model, which typically does not have access to sub-word information, we also concentrate on examining features which apply at or above the word level.

Speaker differences will be evident when examining acoustic-prosodic parameters of speech from multiple speakers. For example, F0 of female speech is substantially higher than that of male speech. In our training data, the mean F0 value of speech from the female speakers was $175 \mathrm{~Hz}$, while the value for male speech was $132 \mathrm{~Hz}$. This difference is meaningless for the purposes of classifying prosodic events, and will likely hinder the use of any direct representation of prosodic parameters. Two simple options available to account for these differences are to either normalise the data, or use speaker-specific parameters. We have opted for the former approach, as there is only very limited data available for some speakers (M3B in particular) in our training set. This also allows for a more generalised approach which can be applied to speech from any additional speakers. The specifics of the normalisation approaches will be described in more detail in the subsections pertaining to each type of feature.

\subsubsection{Duration}

Extracting word duration information is relatively straight-forward. Durations for the training data are obtained by performing a forced alignment using the Viterbi decoder. During the recognition phase, the durations of hypothesised words can also be provided by the decoder.

The raw word duration values can be normalised to account for speaking rate differences. The magnitude of lengthening or shortening due to speaking rate is dependent on the speech sound category, with vowels generally displaying greater variation than consonants. This results in a non-linear relationship between speaking rate and word durations. One possible normalisation technique is to scale the duration of each word based on the identity of the underlying phonemic sequence. This approach is taken by Batliner et al. [156]. In their experiments, though, normalised duration features of this form were found to perform worse than absolute durations in both accent and boundary classification. Through an examination of the interaction between duration and syntactic features, they concluded that there was some structural information relating to a word's syntactic role which was being lost by performing the phoneme-dependent normalisation. Additionally, the speaking rate was estimated locally from a short window surrounding the analysed word. In a word classification experiment, Gadde [124] found gains from normalisation based 
on globally calculated rates of speech to be more consistent than hypothesis-level normalisation. Given these results, we adopt a similar scheme to Gadde, and compensate for speaking rate differences using values calculated over the entire training set.

While it may be desirable to normalise the duration of each phoneme individually, in our experiments, we have assumed that the phonemic sequences of words are not known. Therefore, normalisation must be performed without this information. To evaluate how well a word-based normalisation technique performs compared to a similar phone-based approach, we can measure the Kullback-Leibler distance, as defined by Equation (4.10), between the normalised duration distributions. For the training data, we calculated, for each speaker, the mean durations and standard deviations for every word and phoneme. Phoneme durations were obtained automatically by using the decoder to perform a forced alignment of the data, and recording its state path. The average of the phoneme durations over all speech for a given speaker was also used as a measure of that speaker's global rate of speech. These speaker-dependent means and standard deviations were then used to perform z-score normalisation of the word durations. Three normalisation approaches have been examined.

Word: Durations are normalised based on word identity. $d_{r}(w)$ is the raw duration of word $w, \mu_{s, w}$ is the average duration of word $w$ for speaker $s$, and $\sigma_{w, s}$ is the standard deviation of the duration of $w$ when spoken by $s$.

$$
d_{w}(w)=\frac{d_{r}(w)-\mu_{s, w}}{\sigma_{s, w}}
$$

Phone: Durations are normalised based on the identities of the $n$ phonemes comprising word $w \cdot d_{r}\left(p_{i}\right)$ is the raw duration of the $i^{\text {th }}$ phone in $w, \mu_{s, p}$ is the average duration of phone $p_{i}$ for speaker $s$, and $\sigma_{s, p}$ is the standard deviation of these phones.

$$
d_{p}(w)=\frac{1}{n} \sum_{i=1}^{n} \frac{d_{r}\left(p_{i}\right)-\mu_{s, p}}{\sigma_{s, p}}
$$

Global: Similar to the previous phone normalisation, except only a single mean and standard deviation per speaker are used. These are estimated from the whole training set. $d_{r}\left(p_{i}\right)$ is the raw duration of phone $p_{i}, \mu_{s}$ is the average phoneme duration, and $\sigma_{s}$ is the standard deviation of phone durations for all phones spoken 


\begin{tabular}{ccc}
\hline Global & Phone & Word \\
\hline \hline 0.274 & 0.236 & 0.177 \\
\hline
\end{tabular}

TABLE 6.1: Average KL distances between word duration distributions for three different normalisation schemes.

by speaker $s$.

$$
d_{g}(w)=\frac{1}{n} \sum_{i=1}^{n} \frac{d_{r}\left(p_{i}\right)-\mu_{s}}{\sigma_{s}}
$$

These normalised durations therefore provide estimates, at varying levels of granularity, of how much the duration of a word differs from its mean, after compensating for speaking rate. Table 6.1 shows the KL distances between word duration distributions in the training set for the three normalisation methods. This was calculated as follows: First, the KL distance for each word was calculated as the average pairwise distance between a chosen speaker, and all other speakers. The KL distance over the corpus was then taken to be the average of the word-specific KL distances, weighted by the frequency of the word. Formally, this is:

$$
D_{\text {avg }}=\frac{1}{n} \sum_{w} C(w) \frac{1}{m} \sum_{i} D\left(p \| q_{i}\right)
$$

where $n$ is the number of samples, $C(w)$ is the number of occurrences of word $w, p$ is the normalised duration distribution of word $w$ for a reference speaker (we used F1A) and the $q_{i}$ are normalised duration distributions of $w$ for the remaining $m$ speakers.

The worst performing method, according to these results, is the global normalisation. Because this method only scales durations based on a single mean and standard deviation calculated over the entire data, it cannot account for phoneme or word specific differences between speakers. An example of this is illustrated in Figure 6.1. This shows the duration distributions for the word state, spoken by two speakers. Of the 6 speakers in the data, F2B had the slowest average speech rate, at 14.0 phonemes/s, while M1B had the highest, at 15.2 phonemes/s.

The global and phone based approaches exhibited similar behaviour, and there is a noticeable difference in the means of the distributions between the two speakers. A breakdown of the duration means and standard deviations of each of the underlying phones of state, which is composed of the sequence $\{/ \mathrm{s} /, / \mathrm{t} /, / \mathrm{ey} /, / \mathrm{t} /\}$, for the two speakers is shown in Table 6.2. The values for these phones, when they appear in the context of the word state, are also shown. 


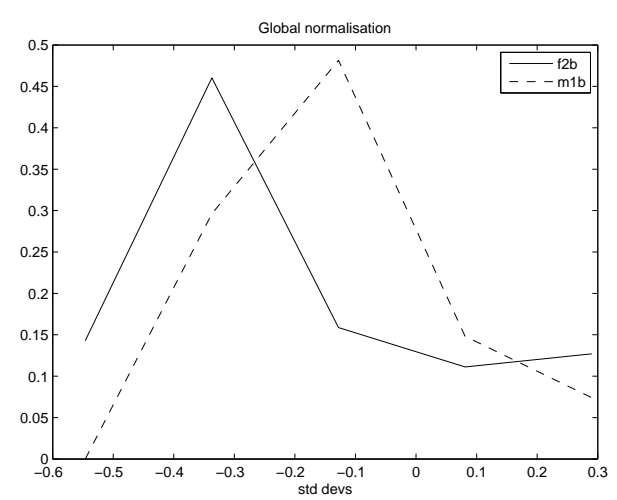

(a) Global normalisation

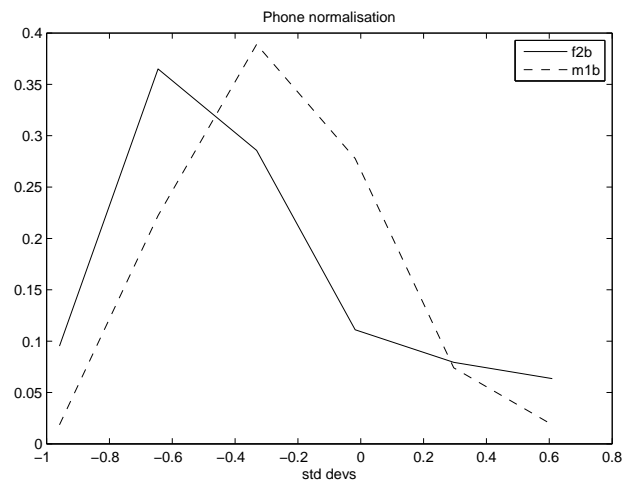

(b) Phone normalisation

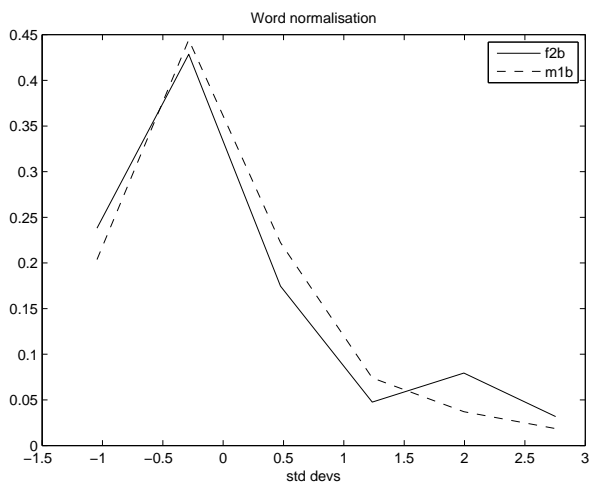

(c) Word normalisation

Figure 6.1: Speaker-dependent duration PDFs for the word state.

The higher speech rate of speaker M1B is reflected in the lower mean durations. The global normalisation approach used a single mean and standard deviation value per speaker, shown in the last row of Table 6.2, to normalise all phone durations. How well this method normalises durations at the word level, then, is highly dependent on the phone composition of the given word. For a word with a low proportion of vowels, which typically have durations that are greater than the average, normalisation using the corpus averages would tend to over-adjust the durations. This results in a distribution with a negative mean, as can be seen in Figure 6.1(a). This effect is particularly prominent for speaker F2B, where the average phone duration over the corpus is substantially larger than the average durations of phones within the word state. There are also some words for which F2B's speech rate, at the word level, was faster than that of M1B. In these instances, applying the global 


\begin{tabular}{ccccc}
\hline & \multicolumn{2}{c}{ all words } & \multicolumn{2}{c}{ state } \\
Phone & f2b & m1b & f2b & m1b \\
\hline \hline$/ \mathrm{s} /$ & $0.098(0.033)$ & $0.081(0.029)$ & $0.091(0.021)$ & $0.092(0.027)$ \\
$/ \mathrm{t} /$ & $0.045(0.027)$ & $0.036(0.025)$ & $0.018(0.008)$ & $0.013(0.006)$ \\
/ey/ & $0.134(0.068)$ & $0.126(0.042)$ & $0.124(0.039)$ & $0.129(0.025)$ \\
\hline average & $0.072(0.052)$ & $0.066(0.055)$ & $0.061(0.050)$ & $0.059(0.050)$ \\
\hline
\end{tabular}

TABLE 6.2: Means and standard deviations (in brackets) of z-score normalised phone durations for the three phonemes in the word state. Scores for these phones, averaged over the entire data set, are shown on the left side, while the right side only considers those phones which occur in the context of state. The bottom row shows the average means and standard deviations for all phones.

normalisation would result in an increased distance between the distributions. This occurred for roughly a third of the vocabulary, and accounted for $17 \%$ of the data. As a result this approach provides little or no benefit for a considerable subset of the data.

Phone-dependent normalisation improved on the performance of the global normalisation. Unlike the global normalisation approach, the phone-dependent normalisation is able to account for duration differences in speech sound categories between speakers. By modelling durations of the underlying phonemes, it can also take into account the non-linear effect speaking rate has on word durations. Despite these advantages, this approach still under-performs the word-based normalisation. Figure 6.1(b) shows that, for the word state, the impact of this type of normalisation is very similar to that of the global normalisation. Rather than being limited by the single mean and variance, though, the main source of error lies with the differing behaviour of the phoneme $/ \mathrm{t} /$ in this word compared to the more general case. Durations of /t/, spoken in state, are much shorter than the average. Again, this results in an inability to appropriately characterise mean durations.

Another reason for the inability to improve on the word normalisation result is practical rather than theoretical. Because the phoneme duration statistics were calculated using automatically aligned data, it is likely to contain segmentation errors. A normalisation scheme based on phonemic information is highly sensitive to such errors, and this contributes to the poor performance of this type of normalisation. Therefore, while this approach may work well in theory, its need for accurate segmental alignments will limit its benefit in some applications. Phoneme durations are also affected by their surrounding phones, which may hinder estimation of normalising parameters. This, however, can be addressed by the use of context-dependent phonemes. Having separate acoustic models for stressed and unstressed vowels, as 
done by Wightman et al. [157], could also improve the automatic segmentation.

Of the three normalisation techniques, the word-based approach gave the best results. This approach allows more detailed information about individual word characteristics to be used than the global normalisation, while being less susceptible to automatic alignment errors than phoneme normalisation. It can also be performed using only word duration information, which is more readily obtainable than the durations of underlying phonemes. One disadvantage of this approach is the potential lack of training data. Duration estimates based on observations of rarely occurring words are likely to be unreliable. To overcome this, means and standard deviations of low count words can be estimated from the phonemes of the most common pronunciation variant, and stored as word-based parameters. This alleviates the need to have the phoneme sequence during recognition. Word-based normalisation of durations will be used for the remainder of this work. Another drawback is that the location of lengthening within a word is lost. This information would be useful, in particular, for identifying the phrase-final lengthening that occurs in the final syllable.

\subsubsection{Fundamental frequency}

Fundamental frequency (F0) is closely related to pitch. To represent pitch information, we use automatically extracted F0 values provided by the Entropic XWaves get_f0 tool. This is an implementation of Talkin's pitch tracking algorithm [54], and calculates the following data at intervals of $10 \mathrm{~ms}$ :

- F0

- Probability of voicing

- RMS energy

A number of post-processing steps, similar to those described by Taylor [97], are performed on this raw pitch contour. Firstly, a 7 point median filter is applied to eliminate any clear pitch tracking errors. Small perturbations in the F0 contour, which are unimportant in prosodic analysis [158], are also removed by this filtering. Ideally, this process should retain the macroscopic movements in the contour. To obtain a continuous pitch contour, which is a more desirable representation of intonation, unvoiced regions are linearly interpolated. The continuous pitch contour is smoothed using a 15 point median filter and finally, normalisation is performed according to the following:

$$
F 0_{z}(t)=\frac{F 0(t)-\mu}{\sigma}
$$



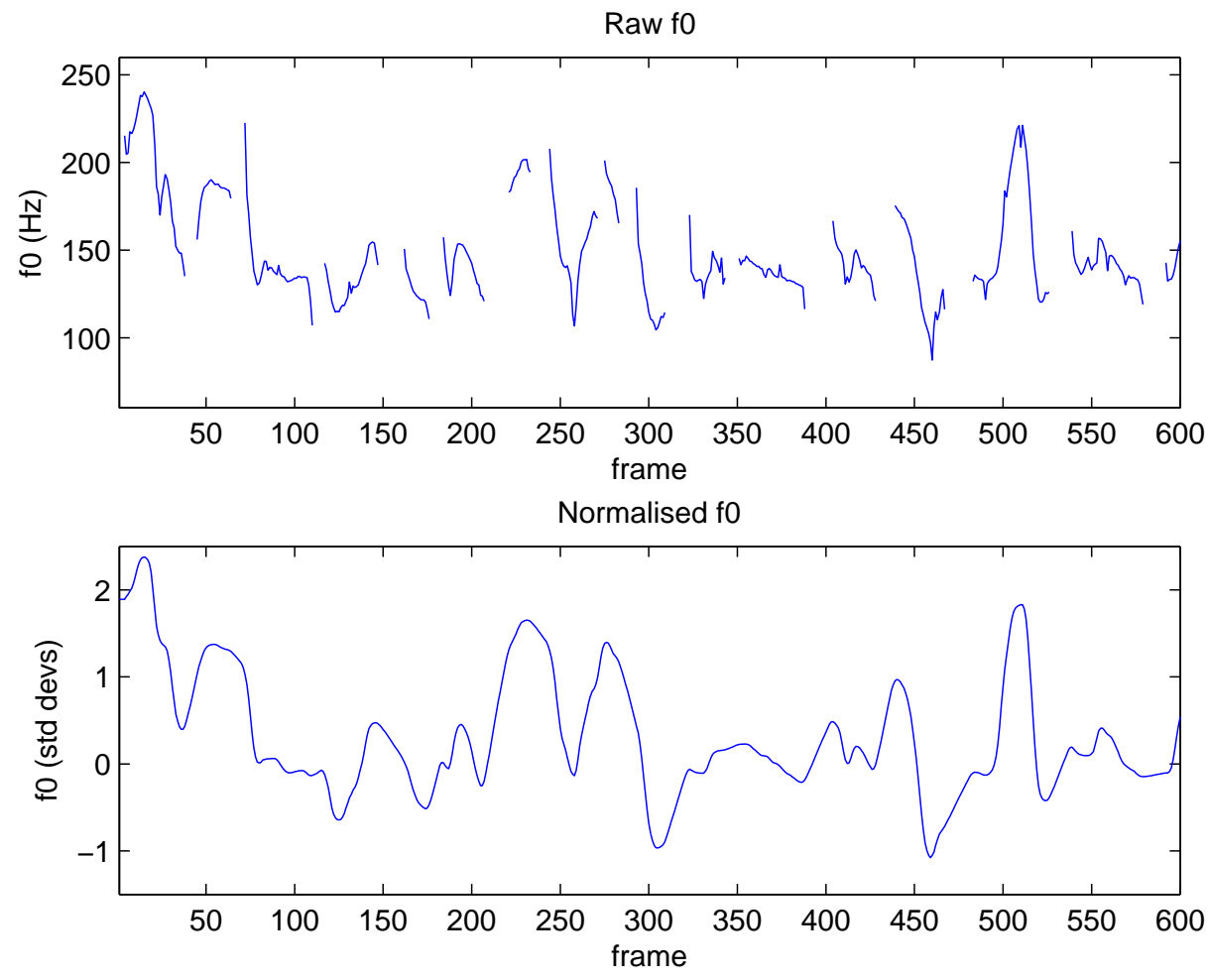

Figure 6.2: Post-processing of an F0 contour. The raw F0 contour extracted by get_fo (top) is median filtered to remove small pitch fluctuations, and unvoiced regions are linearly interpolated. The contour is smoothed with another median filter and finally, z-score normalisation is performed (bottom).

where $F 0(t)$ is a smoothed pitch value at time $t$, and $\mu$ and $\sigma$ are the mean and standard deviation, respectively, of pitch values calculated over all speech frames from the utterance's speaker. Figure 6.2 shows both the original F0 contour extracted by get_fo and the final F0 contour after the post-processing steps have been applied.

From these automatically extracted F0 contours, many different representations can be derived. We are interested in the relationship between F0 and words, so we analyse regions of the contour defined by word boundaries. From each region of the F0 contour associated with a given word, the following real-valued features have been extracted:

1. Minimum - the minimum F0 value.

2. Minimum offset - the temporal offset, from the beginning of the word, of the F0 minimum. This is specified as a percentage of the raw duration of the word.

3. Mean - the mean F0 value. 


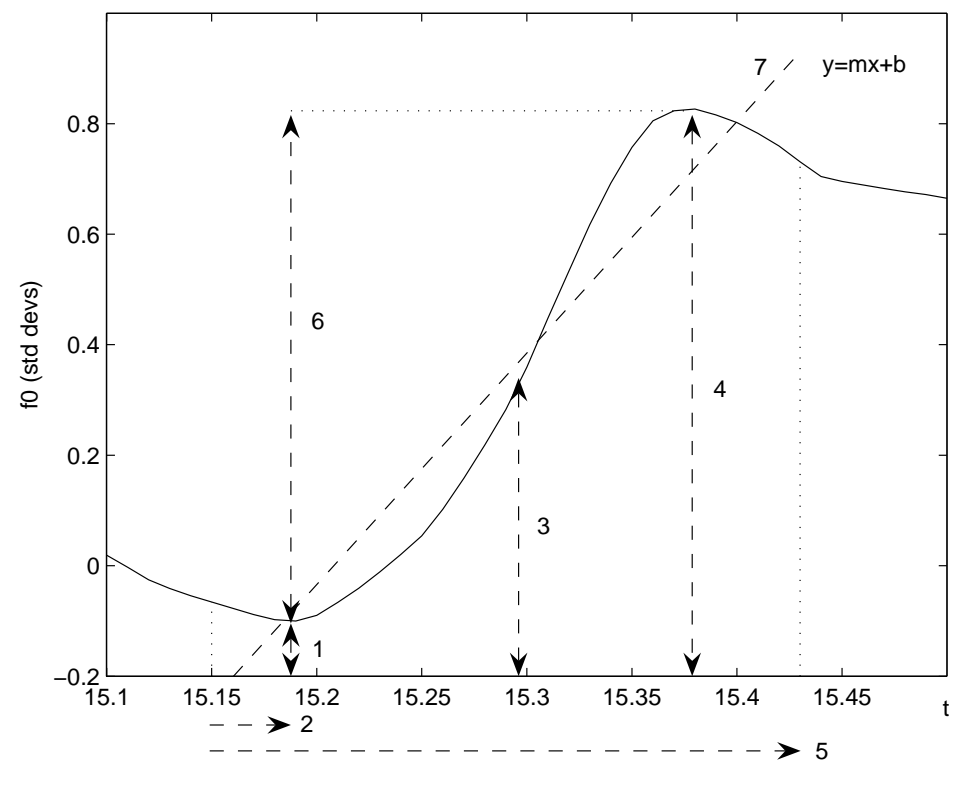

FiguRE 6.3: F0 features derived from the automatically extracted pitch contour. Dotted vertical lines indicate word boundaries.

4. Maximum - the maximum F0 value.

5. Maximum offset - the temporal offset of the F0 maximum.

6. Range - the difference between the maximum and minimum values.

7. Slope - the gradient of the least squares regression line fitted over the specified region.

8. Voicing percentage - the proportion of frames which are voiced.

These features are illustrated in Figure 6.3. There is likely some redundancy in this set of features; however, for this analysis, we are primarily interested in a complete description of the contour rather than obtaining a compact representation.

A potential shortcoming of the previously described features is their very fine granularity. Typically, it is variations such as rises and falls in these values which are indicative of prosodic events, rather than the actual values themselves. Small differences may not only be meaningless, but could also introduce errors into a classifier. Therefore, these features naturally lend themselves to some form of quantisation. Here, we propose to extend the feature set with some additional discrete features based on the following identities: 
- Rise - a sequence of at least 5 consecutive frames $(50 \mathrm{~ms})$ having a gradient greater than a given threshold.

- Fall - a sequence of at least 5 consecutive frames having a gradient lower than a given threshold.

- Peak - a point which lies between a rising segment on the left, and a falling segment on the right. If a peak exists, the surrounding regions are not considered to be rises or falls.

- Trough - a point lying between a falling segment on the left and a rising segment on the right. This is the opposite of a peak.

Features are then defined as the counts of each of these patterns in the region of the F0 contour specified by the given word boundaries. These features attempt to more concisely represent meaningful patterns in the F0 contour. Accentuation is associated with syllables, and extracting features such as minima or maxima directly from the contour over word regions may give inconsistent results. Polysyllabic words, in particular, could interfere with the reliability of those features. By identifying and counting occurrences of specific patterns in a given region, on the other hand, the discrete features should be able to better handle these cases. The threshold for the gradient used in the discrete features was chosen to be \pm 0.1 standard deviations per frame. While it is certainly possible for both the gradient threshold and number of frames defining an event to be tuned, the precise methods by which this can be done, and the criteria to optimise, is not obvious. We have therefore simply left these as fixed values.

\subsubsection{Energy}

The F0 extraction tool, get_f0, provides RMS energy estimates in addition to F0. Energy is closely related to perceived loudness, so the same extraction and postprocessing steps used for F0 are applied here. Similarly, the same set of features are extracted from the energy contour, with the exception of the voicing feature as it is not applicable in the case of energy. 


\begin{tabular}{ccccc}
\hline Accent/Break index & $\mathrm{u} / 1$ & $\mathrm{u} / 4$ & $\mathrm{a} / 1$ & $\mathrm{a} / 4$ \\
\hline \hline Raw duration & $0.18(0.12)$ & $0.43(0.15)$ & $0.38(0.15)$ & $0.53(0.16)$ \\
Normalised duration & $0.26(1.28)$ & $2.52(1.74)$ & $1.88(1.58)$ & $3.39(1.67)$ \\
\hline
\end{tabular}

TABLE 6.3: Means (and standard deviations) of raw and normalised word durations for each of the four prosodic classes.

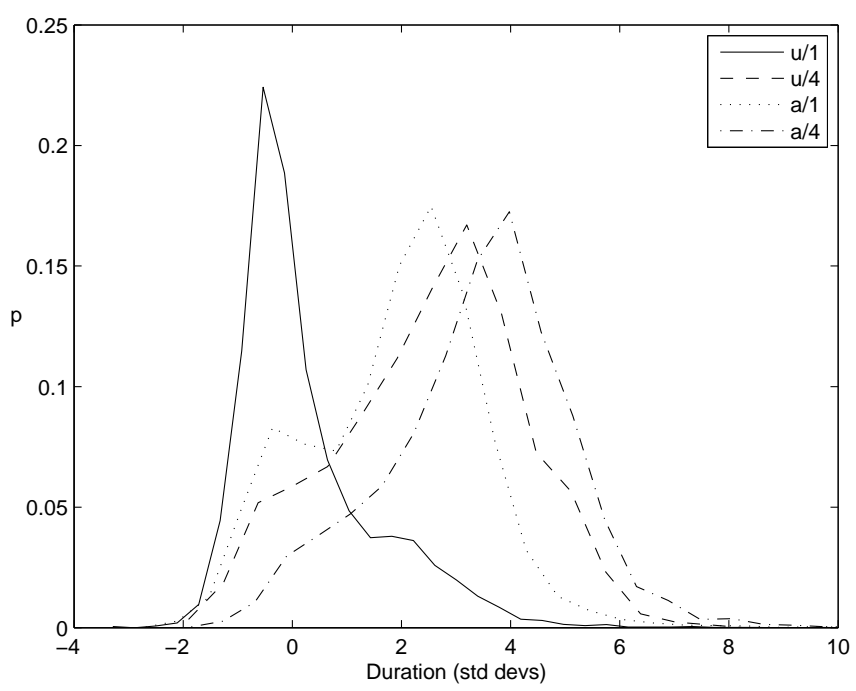

FiguRE 6.4: Distribution of normalised word durations for the four prosodic classes.

\subsection{Feature Analysis}

\subsubsection{Duration}

Durational information is a fairly obvious indicator of prosodic events. Accentuation is associated with increased duration, and phrase boundaries are commonly preceded by a lengthening of the final stressed syllable, as well as pausing at the boundary. Pauses are already modelled in the acoustic and language models, however, so we have excluded it from this analysis. To investigate the effect the presence of these prosodic events have on word durations, we examine their normalised durations under four types of prosodic conditions: unaccented non-phrase-final $(u / 1)$, unaccented phrase-final $(u / 4)$, accented non-phrase-final $(a / 1)$ and accented phrase-final $(a / 4)$. Because a word-based z-score normalisation was used, the comparison across words with differing raw durations is still meaningful. These results are summarised in Table 6.3.

The z-score results indicate that both accented words and phrase-final words are 


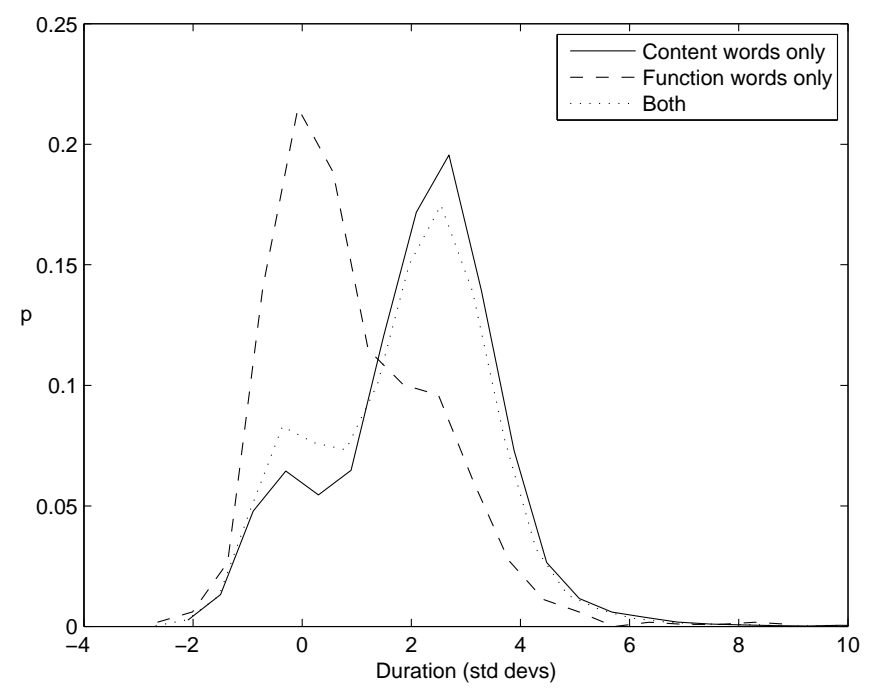

FiguRE 6.5: Distribution of normalised durations for accented phrase-final words, separated by POS. Content words exhibit the characteristic pre-boundary lengthening but function words are largely unaffected.

characterised by greater durations. Words which were both accented and phrasefinal exhibited significantly higher mean durations than words with other combinations of accents and boundaries. Lengthening due to the presence of a boundary after the word increased mean durations more than accentuation did; however, standard deviations in all cases were quite large. The same trends were mirrored by the raw duration values. Because these are influenced by word identity, with short function words unlikely to be accented or occur at the end of a prosodic phrase, there is slightly less separation between their distributions. The distributions of the normalised data, displayed in Figure 6.4, show that duration information alone is capable of providing a reasonably good degree of discrimination between words having both accents and boundaries, and those with neither. Durations of words which were either accented or phrase-final, but not both, lay somewhere in between the two extremes. Interestingly, the duration distribution of those words which were accented but not phrase-final exhibited a secondary peak located near that of the unaccented words. This warrants closer inspection, as it indicates that the lengthening associated with accentuation does not apply uniformly to all words.

Examination of the duration distributions of the accented words, conditioned on their POS tags, revealed two fairly distinct groupings, roughly corresponding to function and content words. Here, content words were considered to be nouns, verbs, adjectives and adverbs, while the remaining words were classed as function 
words. This distinction is illustrated in Figure 6.5, in which the distribution of accented, non-phrase-final words has been further partitioned based on their POS labels. It can be seen that introducing this distinction largely accounts for the two dominant peaks that appear in the combined distribution. This highlights the importance of considering syntax in investigations of prosody, as the two are closely related. Individually, POS and duration information both provide useful cues to determining the prosodic state of a given word. Using both knowledge sources in conjunction, though, can further improve the discriminative ability through their interaction. This, in fact, is similar to the results of Section [5.2, in which positive interaction information was found between word identity, prosodic information and POS.

\subsubsection{F0 and RMS energy}

Results of a similar analysis of the real-valued voicing, F0 and energy features are presented in Table 6.4. The values in the first row, showing the percentage of voiced frames in a word, are slightly higher for accented words than their unaccented variants. Accentuation tends to increase a word's duration, and typically it is the durations of (voiced) vowels which undergo the greatest lengthening. This explanation, however, does not account for the contrary behaviour observed with the phrase-final words. For both accented and unaccented words, the percentage of voiced frames was lower when the word was followed by a boundary than when there was no boundary. Boundaries generally cause a lengthening of the preceding word, and the statistics in Table 6.3 indicate the magnitude of this lengthening is even greater than that of accentuation. The pre-boundary lengthening, then, would appear to affect unvoiced sounds to a greater degree than lengthening caused by accentuation. It is also possible that the occurrence of phonemes in the last syllable of phrase-final words, where lengthening is most pronounced, is skewed towards unvoiced sounds. In our data, the high frequency of the unvoiced fricative /s/ in these syllables could contribute to the reduced percentage of voiced frames.

The results for the slope feature were almost identical irrespective of the prosodic events associated with the given word. Additionally, the low standard deviations of this feature suggest that the values are consistently very small. A likely explanation for this is that a regression line can be quite a poor fit of the data, even if its purpose is only to be an estimate of the gradient. A common realisation of the $\mathrm{H}^{*}$ pitch accent involves a rise-fall movement of the pitch contour, such as that shown in Figure 6.6. The linear approximation to this type of data would completely fail to 


\begin{tabular}{ccccc}
\hline Accent/Break index & $\mathrm{u} / 1$ & $\mathrm{u} / 4$ & $\mathrm{a} / 1$ & $\mathrm{a} / 4$ \\
\hline \hline Voicing & & & & \\
\% voiced & $0.72(0.26)$ & $0.62(0.20)$ & $0.72(0.19)$ & $0.68(0.18)$ \\
\hline F0 & & & & \\
min & $-0.43(0.82)$ & $-1.53(1.00)$ & $-0.36(0.83)$ & $-1.31(0.92)$ \\
min offset & $0.52(0.38)$ & $0.54(0.28)$ & $0.46(0.43)$ & $0.65(0.32)$ \\
mean & $-0.10(0.68)$ & $-0.70(0.70)$ & $0.38(0.64)$ & $-0.15(0.58)$ \\
max & $0.21(0.71)$ & $0.21(0.75)$ & $0.87(0.73)$ & $0.80(0.71)$ \\
max offset & $0.40(0.40)$ & $0.40(0.43)$ & $0.49(0.27)$ & $0.39(0.27)$ \\
range & $0.64(0.67)$ & $1.74(1.00)$ & $1.23(0.85)$ & $2.11(1.01)$ \\
slope & $0.03(0.07)$ & $0.03(0.04)$ & $0.03(0.04)$ & $0.03(0.03)$ \\
\hline RMS & & & & \\
min & $-0.48(0.70)$ & $-1.04(0.62)$ & $-0.69(0.66)$ & $-1.01(0.59)$ \\
min offset & $0.51(0.38)$ & $0.62(0.37)$ & $0.45(0.38)$ & $0.62(0.39)$ \\
mean & $0.15(0.50)$ & $-0.03(0.45)$ & $0.33(0.42)$ & $0.20(0.40)$ \\
max & $0.56(0.46)$ & $0.58(0.39)$ & $0.90(0.35)$ & $0.89(0.35)$ \\
max offset & $0.42(0.32)$ & $0.35(0.28)$ & $0.45(0.22)$ & $0.37(0.20)$ \\
range & $1.04(0.68)$ & $1.63(0.53)$ & $1.59(0.63)$ & $1.91(0.54)$ \\
slope & $0.02(0.09)$ & $0.02(0.04)$ & $0.02(0.04)$ & $0.02(0.03)$ \\
\hline \hline
\end{tabular}

TABLE 6.4: Means (and standard deviations) of voicing, F0 and RMS energy features for the four combinations of pitch accent and break index.

capture any relevant information about the contour, instead giving a gradient which is close to zero. Another reason is that there may be more than one pitch movement associated with polysyllabic words. In these cases, the regression line can only provide an estimate of the net movement in F0 across the entire word. Therefore, the average gradient of the F0 contour using a word-based analysis window often does not contain any meaningful information.

The features sampled directly from the contour displayed varying properties depending on their prosodic class. Pitch accents are often associated with local peaks in the F0 contour, and this is reflected in the higher F0 mean and maxima of accented words. The equivalent energy features also were greater for these words. Minimum F0 and energy values were not significantly affected by a word's accentuation. Boundaries, on the other hand, did decrease the average values of the F0 and energy minima, and this is consistent with the final lowering observed in declarative speech [159]. The lowering in words preceding boundaries is also evident in the mean results, which was generally lower for unaccented phrase-final words than other types. Mean values for unaccented non-phrase-final and accented phrase-final words were quite similar due to the opposing influences of boundaries and accents. 


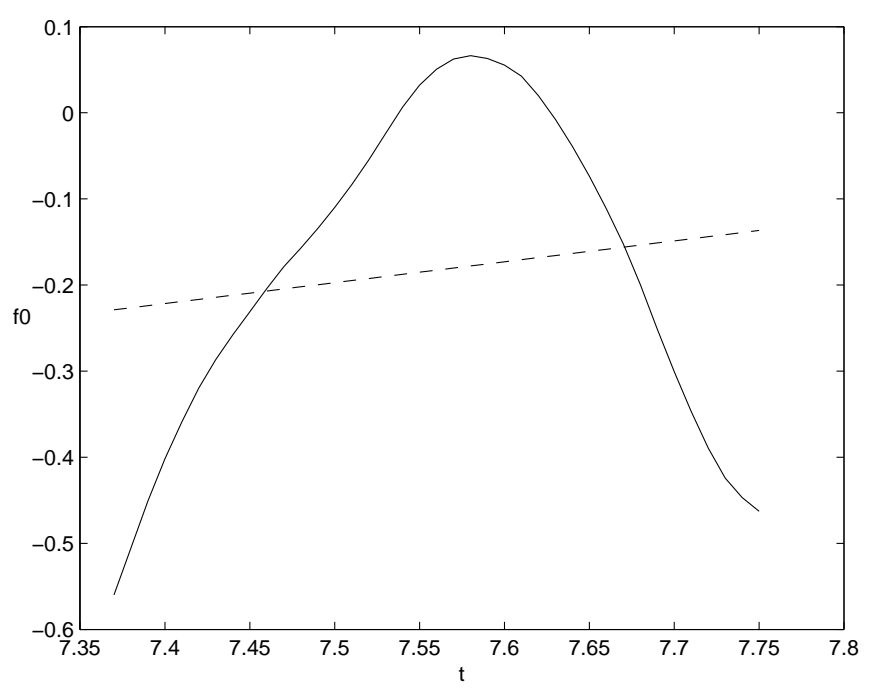

FiguRE 6.6: A typical rise-fall F0 pattern occurring during the word reaches, and the (dotted) least squares regression line fitted over the region.

While F0 values tend to fall in the presence of a boundary, accentuation is typically associated with a local maximum. The combination of these two effects results in means which are not much different from that of words without any prosodic event. The actual contours themselves, though, are not necessarily similar. This can be seen in the differences in the minimum and maximum values for each combination of prosodic events. The range values too, which increased with both accents and boundaries, support this interpretation. In the case of accents, this is largely due to the increase in maxima caused by sharp pitch and energy rises, while the decreased minima caused by final lowering similarly contributes to an increase in the range over the region of the word.

The two offset features, specifying the times at which the minima and maxima occur within the word have a relatively small range between the classes. In unaccented words, there was no observable difference in these offset values. Accented words displayed greater variability, with minima occurring later if the word was phrase-final, and earlier otherwise. F0 maxima also tended to occur later for accented phrase-final words. While these features do have lower standard deviations in comparison to the other feature types, giving them increased reliability, on the whole they provide only minimal beneficial information.

The RMS slope feature, like its F0 equivalent, did not provide much discriminatory information, probably for the same reasons. The remaining features behaved similarly to the F0 features, but exhibited smaller variation in general. There is a 


\begin{tabular}{ccccc}
\hline Accent/Break index & $\mathrm{u} / 1$ & $\mathrm{u} / 4$ & $\mathrm{a} / 1$ & $\mathrm{a} / 4$ \\
\hline \hline F0 & & & & \\
rise & $0.26(0.45)$ & $0.66(0.51)$ & $0.34(0.50)$ & $0.50(0.54)$ \\
fall & $0.35(0.49)$ & $0.39(0.55)$ & $0.66(0.51)$ & $0.57(0.56)$ \\
peak & $0.05(0.22)$ & $0.16(0.39)$ & $0.55(0.53)$ & $0.73(0.51)$ \\
trough & $0.06(0.25)$ & $0.58(0.53)$ & $0.15(0.38)$ & $0.52(0.57)$ \\
\hline \hline RMS & & & & \\
rise & $0.21(0.42)$ & $0.22(0.45)$ & $0.28(0.47)$ & $0.17(0.40)$ \\
fall & $0.50(0.55)$ & $1.02(0.64)$ & $0.90(0.64)$ & $1.22(0.65)$ \\
peak & $0.19(0.43)$ & $0.71(0.70)$ & $0.75(0.66)$ & $1.04(0.67)$ \\
trough & $0.11(0.33)$ & $0.54(0.64)$ & $0.44(0.61)$ & $0.60(0.69)$ \\
\hline
\end{tabular}

TABLE 6.5: Means (and standard deviations) of quantised F0 and RMS energy features for the four combinations of pitch accent and break index.

strong positive correlation between these F0 and energy features, with classes which were characterised by higher F0 invariably having higher energy. This is to be expected, as both types of prosodic events often involve changes in F0 and energy occurring in the same direction. Like their F0 counterparts, the RMS offset features also provided little discriminative information.

In contrast to the poor discriminative ability of the slope feature, the quantised F0 rise and fall features demonstrated substantially different behaviour depending on prosodic class. These results, as well as those for the peak and trough features, are shown in Table 6.5. This shows a number of differences between F0 and energy. While this set of F0 features was quite good at capturing the accented versus unaccented and boundary vs non-boundary words, the equivalent energy features provided less distinction. This approach, therefore, seems to be an appropriate way of encoding F0 information. In the case of energy, the discrimination provided by these features are less evident. Because the energy contour is influenced to a greater degree by segmental identity, its correlation to prosodic events is weaker. Locating specific patterns in this contour, then, is of less value than it is for F0; however, they still contribute some useful information.

As expected, the ability to identify the rise-fall and fall-rise patterns, particularly in F0 contours, was useful for separating words of differing prosodic classes. F0 peaks, especially, showed quite distinct values for every class. In general, accented words contained considerably more peaks than unaccented words, and the occurrence of troughs was increased by the presence of a phrase boundary. It is also important that these particular F0 patterns be distinguished from simple rises and falls, which the results indicate are affected differently by accents and boundaries. By counting 
occurrences of these specific patterns rather than attempting to fit a function to the whole contour segment, these features also provide more meaningful results when there is more than a single intonational event in the region under consideration. This is particularly useful when dealing with polysyllabic words, which may have multiple weaker accents in addition to the primary accent.

\subsection{Summary}

In this chapter, we have described methods for extracting duration information and fundamental frequency and RMS energy contours. These are all highly speaker dependent, and an important consideration in their use is normalisation. For duration, we have found a simple word-based z-score normalisation to perform well, despite it being unable to capture the non-linear effect that speaking rate has on word durations. Phoneme-based normalisation, while theoretically superior, was hindered by the use of automatic segmentation and the lack of lexical stress modelling in our acoustic models. Z-score normalisation was also used to normalise for speakerspecific means and dynamic ranges of $\mathrm{F} 0$ and energy values. This normalisation was performed after post-processing the contours to smooth and interpolate them.

Representing all values in terms of standard deviations from their means allows for a comparison of the normalised values across different words. A number of features were derived from the duration information and F0 and energy contours. In addition to the real-valued features obtained directly from these sources, we also proposed a quantisation scheme for the F0 and energy contours. These quantised features were designed to identify specific patterns in the contours, and could be used to produce meaningful statistics for polysyllabic words that potentially contained more than a single prosodic event.

An analysis of the relationship between the acoustic-prosodic parameters and the occurrences of accents and boundaries in words showed a number of correlations. The most relevant features for distinguishing between the prosodic classes, and their relationship to these classes, are summarised in Table 6.6. Most of the features examined here contributed some useful information to the distinction between events, though there is significant redundancy in the full feature set. Of particular note are the results of the quantised features, which were very effective at characterising differences in the F0 contours associated with the various prosodic events. This is largely due to the explicit identification of the rise-fall and fall-rise patterns which commonly occur in the F0 contour. The final set of duration, F0 and energy features should serve to provide good discrimination between the prosodic classes. 


\begin{tabular}{ccccc}
\hline Accent/Break index & $\mathrm{u} / 1$ & $\mathrm{u} / 4$ & $\mathrm{a} / 1$ & $\mathrm{a} / 4$ \\
\hline \hline raw duration & $\mathrm{L}$ & & & $\mathrm{H}$ \\
normalised duration & $\mathrm{L}$ & & & $\mathrm{H}$ \\
\% voiced & & $\mathrm{L}$ & & \\
F0 min & & $\mathrm{L}$ & & $\mathrm{L}$ \\
F0 mean & & $\mathrm{L}$ & $\mathrm{H}$ & \\
F0 max & & & $\mathrm{H}$ & $\mathrm{H}$ \\
F0 range & $\mathrm{L}$ & $\mathrm{H}$ & & $\mathrm{H}$ \\
F0 rise & & $\mathrm{H}$ & & $\mathrm{H}$ \\
F0 fall & & & $\mathrm{H}$ & $\mathrm{H}$ \\
F0 peak & & & $\mathrm{H}$ & $\mathrm{H}$ \\
F0 trough & & $\mathrm{H}$ & & $\mathrm{H}$ \\
RMS min & & $\mathrm{L}$ & & $\mathrm{L}$ \\
RMS mean & & $\mathrm{L}$ & & \\
RMS max & & & $\mathrm{H}$ & $\mathrm{H}$ \\
RMS range & $\mathrm{L}$ & & & $\mathrm{H}$ \\
RMS rise & & & $\mathrm{H}$ & \\
RMS fall & $\mathrm{L}$ & & & $\mathrm{H}$ \\
RMS peak & $\mathrm{L}$ & & & $\mathrm{H}$ \\
RMS trough & $\mathrm{L}$ & & & \\
\hline
\end{tabular}

TABLE 6.6: Relationship between prosodic events and automatically extracted features. Entries for which a feature is useful for determining the prosodic class are marked as either $\mathrm{H}$ (indicating the mean feature value is higher than average) or $\mathrm{L}$ (lower than average). 


\section{Chapter 7}

\section{Language Modelling with Raw Features}

The language models derived thus far have relied on the presence of manually transcribed prosodic labels. A major drawback of this is the fact that the transcriptions are costly and time-consuming to develop. Even corpora developed for research purposes are very limited in terms of prosodic annotations, and these transcriptions will be unavailable in practice. A language model incorporating prosodic information will therefore have to make use of automatically extracted features to be of any practical benefit. If a small set of prosodically labelled data is accessible, models can be trained to perform automatic transcription. If no such data can be obtained, fully automated procedures need to be considered.

This chapter will describe the construction of language models using both approaches. The first section of this chapter will discuss how non-binary features such as the automatically extracted prosodic features derived in Chapter 6 can be incorporated into maximum entropy models. It will cover the advantages and disadvantages of each of the alternatives, and describe the method to be used in these experiments. The second section will introduce dimensionality reduction techniques, and discuss why they are relevant for the prosodic features. In particular, it will focus on the Locality Preserving Projection and how the properties of this technique are useful for the current task. In the third section, the construction of a maximum entropy model used to annotate word sequences is detailed. This model is also used to transcribe the $N$-best output of a recogniser, which is then rescored by a language model employing ToBI features. The fourth section of this chapter will consider the direct usage of raw prosodic information in the language model. This makes the assumption that no transcribed data is available at all. Model features are derived which can compactly encode both the prosodic state of a given word and its re- 
cent history. The effectiveness of these features are evaluated and compared against manually transcribed features. A summary of the findings will be presented in the final section.

\subsection{Handling Non-Binary Features}

\subsubsection{Real-valued features}

In the maximum entropy models described so far, only binary-valued features have been considered. The prosodic information extracted in Chapter 6, however, consists of both integer and real-valued features. There are a few common approaches to dealing with these types of features. In general, the maximum entropy framework does not preclude the use of real-valued features, and these can simply be included directly into a model. An integer feature $f(h, w)=n$ is equivalent to $n$ occurrences of the binary feature $f(h, w)=1$, with the feature becoming inactive when $n=0$. For our count-based features, namely rises, falls, peaks and troughs, this interpretation is quite sensible. Real-valued features can also be considered to be a further extension of this to non-integer values.

For the special case when the feature values are logarithms of conditional probabilities (i.e. $f_{i}(h, w)=\log p_{i}(w \mid h)$ ), incorporating such features is equivalent to a log-linear combination of the component distributions [160]. The greatest advantage of introducing the additional models $p_{i}(h \mid w)$, is the potential for decreasing the parameter count. A probability distribution defined on a subset of $H \times W$ can be used to encapsulate multiple binary features. Under this approach, the binary features themselves can be interpreted as probability distributions, each having a support of a single event [161]. Using the intermediate distributions allows for information to be incorporated which would otherwise lead to an intractable model.

This approach, however, introduces an additional level of complexity, as it requires the use of an appropriate model $p(h \mid w)$. There is also a problem with the potential loss of information as a result of the reduction of the original feature space into a single probability measure. By performing model combination, rather than feature combination, the maximum entropy model's ability to appropriately weight information is fundamentally restricted. The contributions of individual pieces of evidence are determined by the intermediate model, and are not subject to the maximum entropy criterion. Furthermore, if ML estimation is used to calculate the distribution $p(w \mid h)$, it may also be necessary to consider smoothing. If the context, $h$, provides only limited information on the target to be classified, the probability 
estimates may also be prone to error. In these cases, it may be beneficial to provide the full set of features to the model, thus allowing it to perform feature selection and parameter estimation in conjunction with other model features.

\subsubsection{Quantisation}

An alternative to handling real-valued features is quantisation. Under this approach, all real values falling within a specified range are considered to belong to the same bin, and each bin is treated as a unique binary feature. This can be advantageous if minor differences in the real-valued data are relatively unimportant, as is normally the case with acoustic-prosodic features. Grouping similar values together into a single equivalence class can allow more robust estimates to be obtained if the range of prosodic feature values spanned by the bin have the same influence on the modelled target. Choosing appropriate values for the number of bins, their sizes, and the mapping from real values to bins are an important part of the quantisation process. However these parameters depend on the specific nature of the data, and finding optimal values is generally not a simple task. Selecting too many bins can result in problems of sparsity and over-training, while using too few bins can mask patterns in the data. For the mapping issue, uniform occupancy binning has been found to perform well for spectral features in maximum entropy acoustic models [162].

A major disadvantage of the quantisation approach is the increase in parameter count. Partitioning a real valued feature into $n$ bins results in an additional $(n-1) \times|W|$ possible features to the model. This is a serious issue for maximum entropy models, which have computationally expensive parameter estimation procedures. Another drawback of this approach is the inability to encode the relationship between bins. By binning the features, the model loses information relating to the actual values specified by the bins. For example, instances of a feature $f(h, w)=2$ and $f(h, w)=3$ may be considered to be close, while $f(h, w)=8$ represents a drastic difference. If each of these features belong to separate bins, however, this relationship cannot be captured.

In our experiments, we have chosen to incorporate the non-binary features directly into the model, primarily due to the simplicity of this technique. It also results in fewer parameters than quantisation, and does not require selection of binning parameters. Although using probabilities as features can further decrease the number of parameters, we believe allowing the full level of feature interaction to be preferable. We will investigate other methods for reducing the parameter count in the next section. 


\subsection{Dimensionality Reduction}

The prosody extraction process described in Chapter 6 provides a set of 19 integer and real-valued features describing the prosodic context of each word. Although it is possible to incorporate these directly as model features, there are some disadvantages to doing so. The first is that these features are likely to contain a high level of redundancy. As noted in the previous chapter, a number of these features exhibit very similar patterns with respect to prosodic classes. For instance, high F0 and energy maxima are both correlated with pitch accents. Intuitively, merging these into a single feature could result in a more compact representation with minimal loss of information. In addition, compressing the feature space can provide a generalisation of the original data. The automatically extracted features are subject to a variety of estimation errors. Minor perturbations caused by these inaccuracies can be considered to be noise, and may negatively affect classifier performance. If these effects can be removed or ignored, a more accurate model of the data, and any patterns it exhibits, could be obtained. Even if some useful information is lost by reducing the number of features, this trade-off may be worthwhile if the features are to be used in models, such as the maximum entropy model, which are expensive to train.

To a lesser extent, the curse of dimensionality also motivates the use of lower dimensional features. While the dimensionality of our particular problem is much smaller than that encountered in other NLP problems, such as document clustering, it is heavily reliant on the use of distance metrics as indicators of similarity between features. Increasing the dimension of a space leads to an exponential increase in volume. As a result, points in high-dimensional spaces tend to become equidistant, thus making automatic learning based on distances difficult. For the same reason, the sparsity of a fixed amount of data also increases exponentially, introducing additional problems for statistical models.

\subsubsection{Linear dimensionality deduction}

Dimensionality reduction methods can be broadly categorised into linear and nonlinear approaches. Linear techniques such as Principal Components Analysis (PCA) and Linear Discriminant Analysis (LDA) project data onto subspaces by forming linear combinations of the data vectors. One of the biggest advantages of these approaches is their low computational cost. However, they cannot accurately represent data that are non-linearly embedded in the high dimensional space. Many techniques have been proposed to deal with such data (see van der Maaten et al. [163] for a 
review). These are able to find more complex structures in the data, but suffer from higher computational costs. In addition, while they perform very well for artificial data sets, they often fail to significantly outperform linear techniques on real world data [163]. The subspace mappings generated by some non-linear techniques are also defined only on training data. For applications which require unknown points to be projected onto the subspace, such techniques are inappropriate. This is the case for our task, in which predictions need to be made based on the prosodic context of unseen samples. For these reasons, we will focus only on linear dimensionality reduction methods.

In linear dimensionality reduction, data in the original space are approximated as linear combinations of a set of lower dimensional basis vectors. A set of $d$ dimensional data points, $X=\left\{\mathbf{x}_{1}, \mathbf{x}_{2}, \ldots, \mathbf{x}_{n}\right\}$, can be mapped onto an $r$-dimensional $(r<d)$ subspace through the use of a $d \times r$ transformation matrix $A$ :

$$
\mathbf{y}_{i}=A^{T} \mathbf{x}_{i}
$$

where $\mathbf{y}_{i}$ is the lower dimensional representation of $\mathbf{x}_{i}$. The choice of $A$ can be guided by a variety of criteria, and this choice will affect the resulting data and its properties. PCA, for example, selects the set of orthogonal basis functions that maximises the retention of data variance. Assuming the samples of $X$ are centred about the origin (which can be achieved by subtracting the column means from $X$ ) the linear mapping a is chosen to maximise:

$$
\mathbf{a}^{*}=\underset{\mathbf{a}}{\arg \max } \mathbf{a}^{T} X X^{T} \mathbf{a}
$$

subject to $\mathbf{a}^{T} \mathbf{a}=1$. The principal components forming the columns of $A$ can be calculated as the leading eigenvectors of the sample covariance matrix $X X^{T}$. A transformed sample, $\mathbf{y}=A^{T} \mathbf{x}$, is then the closest approximation to $\mathbf{x}$ in the leastsquares sense.

By retaining the greatest possible variance, PCA provides the optimal linear transformation for data reconstruction. For the purposes of classification, though, this is not necessarily the desired outcome. If multiple classes exhibit strong variations in the same direction, that direction will have a higher corresponding eigenvalue than those directions which provides more discrimination between the classes. Because directions of lower variance are considered to be less important by PCA, potentially useful information may be discarded by selecting only the principal eigenvectors. 


\subsubsection{Locality Preserving Projections}

A more recently proposed approach to linear dimensionality reduction is the Locality Preserving Projection (LPP) [164]. This technique differs to PCA in that it attempts to retain the local structure of the space. In LPP, the optimising criterion requires the minimisation of the objective function:

$$
\mathbf{a}^{*}=\underset{\mathbf{a}}{\arg \min } \sum_{i} \sum_{j}\left(\mathbf{a}^{T} \mathbf{x}_{i}-\mathbf{a}^{T} \mathbf{x}_{j}\right)^{2} S_{i j}
$$

$S_{i j}$ encodes the similarity between points $\mathbf{x}_{i}$ and $\mathbf{x}_{j}$, and the set of these values forms the similarity matrix, $S$. If two points have high similarity, then this function penalises mappings which result in a large distance between their transformations. This ensures that any points which are close to each other in the original space remain close after the projection. The transformation is found by solving the generalised eigenvalue problem:

$$
X L X^{T} \mathbf{a}=\lambda X D X^{T} \mathbf{a}
$$

where $L=D-S$, and the entries of the diagonal matrix $D$ are given by $D_{i i}=\sum_{j} S_{i j}$. $L$ is also the graph Laplacian [165], and the LPP solution is a linear approximation of the non-linear Laplacian Eigenmap technique [166]. The transformation matrix, $A$, comprises the eigenvectors corresponding to the $r$ lowest eigenvalues. In practice, a PCA usually needs to be performed as a pre-processing step to ensure $X D X^{T}$ is non-singular.

The similarity matrix, $S$, is calculated from an adjacency graph of the data. Each node in the graph corresponds to a data point, and node $n_{j}$ is connected to node $n_{i}$ by an undirected arc if the point $x_{j}$ is one of the $k$ nearest neighbours of $x_{i}$ in the original space. An entry in the similarity matrix, $S_{i j}$, is the weight of the arc connecting $n_{i}$ and $n_{j} . S_{i j}$ is zero if the respective nodes are unconnected. Obtaining $S$ therefore requires the following to be defined:

- The similarity metric between two points.

- The number of nearest neighbours, $k$.

- The weighting function for arcs.

To measure the similarity between points, represented as vectors in the original prosody space, we have used the cosine similarity function commonly employed in document clustering and information retrieval applications. The cosine similarity 
between two vectors, $\mathbf{x}_{i}$ and $\mathbf{x}_{j}$, is given by:

$$
\operatorname{cosim}\left(\mathbf{x}_{i}, \mathbf{x}_{j}\right)=\cos \left(\mathbf{x}_{i}, \mathbf{x}_{j}\right)=\frac{\mathbf{x}_{i} \cdot \mathbf{x}_{j}}{\left|\mathbf{x}_{i}\right|\left|\mathbf{x}_{j}\right|}
$$

The choice of $k$ dictates the degree of localisation of the resulting projection. For a small value of $k$, the graph will only capture information within a very limited neighbourhood. A large $k$ incorporates more global structure, and LPP performed using a complete graph bears a resemblance to PCA [167]. Finally, the magnitude of the similarity between two points is encoded as the cosine similarity between their respective points. A number of other approaches for selecting parameters in the construction of the graph, as well as weighting its links, have been investigated by Cai et al. [167].

\subsection{Prosody Labelling}

The language models developed in Chapter 5 required words to be explicitly transcribed with ToBI labels. Because this process involves human input, it will not be available in most cases. In order to be of practical use, then, the data need to be labelled by some unsupervised means. Here, we will investigate the use of the prosodic information extracted in Chapter 6 for the purpose of automatic annotation.

The required task of the automatic transcription system is to take, as input, some context, $h$, and output its prosodic label $p$, while making use of various lexical, syntactic and acoustic-prosodic knowledge sources. The word sequence is assumed to be known, although it may contain errors if recogniser output is used. The possible outputs of our labelling system are the same four prosodic classes, $P=$ $\{u / 1, u / 4, a / 1, a / 4\}$, used in the previous chapter's analyses. The labelling can be formulated as the classification problem:

$$
p=\underset{p \in P}{\arg \max } p(p \mid h)
$$

To perform this classification, we used maximum entropy models trained to calculate $p\left(p_{i} \mid h_{1}, \ldots, h_{i}\right)$. The context at position $i$ is the tuple:

$$
h_{i}=\left(w_{i}, s_{i}, \mathbf{x}_{\mathbf{i}}\right)
$$

where $w_{i}$ is the word to be labelled, $s_{i}$ is its POS label and $\mathbf{x}_{\mathbf{i}}$ is the vector of duration, F0 and energy parameters described in Chapter 6. The feature functions used by these models will be detailed in Section 7.3.2. 


\subsubsection{Baseline model}

Lexical information such as a word's surface form, as well as its POS tag, already provide a significant amount of information about its prosodic class. To examine the additional contribution of the prosodic features, we compare models to a baseline constructed using only this textual information. The baseline model uses the following word and POS feature types:

$$
\begin{aligned}
& f_{w}(h, w)=\left\{\begin{array}{lll}
1 & : & \text { if } w=w_{i} \text { and } w_{i-m}^{i} \in h \\
0 & : & \text { otherwise }
\end{array}\right. \\
& f_{s}(h, w)=\left\{\begin{array}{lll}
1 & : & \text { if } w=w_{i} \text { and } s_{i-m}^{i} \in h \\
0 & : & \text { otherwise }
\end{array}\right.
\end{aligned}
$$

for $0 \leq m \leq 2$. These resemble the standard $n$-gram and POS features used in Chapter 5; however, they differ by also including information obtained from the current word.

\subsubsection{Prosodic model features}

For every word in the training set, an acoustic-prosodic feature vector was constructed according to the procedure documented in Chapter 6. These samples were then projected onto a linear subspace determined by LPP. The similarity matrix used in LPP is very large $(n \times n)$, so only a subset (of approximately 5000 samples) of the data was used to obtain the transformation matrix. The prosodic feature vectors we used contain a diverse set of information derived from varying sources, and some dimensions are more prone to variation than others. We have therefore chosen a fairly high number of nearest neighbours $(k=50)$ when constructing the adjacency graph in order to ensure that it is not localised only to those dimensions with low variance.

The values of the prosodic feature vectors, extracted using the hypothesised boundaries of word $w_{i}$ and projected onto the LPP subspace, $\mathbf{y}_{i}$, were then incorporated into the model as the following maximum entropy features:

$$
f_{l}(h, w)=\left\{\begin{array}{rll}
y_{i l} & : & \text { if } w=w_{i} \\
0 & : & \text { otherwise }
\end{array}\right.
$$

$y_{i l}$ is the value of $\mathbf{y}_{i}$ along the $l^{\text {th }}$ axis for $l=1, \ldots, r$ and where $r$, as defined in Section 7.2.2, is the reduced number of dimensions after performing the LPP. Each feature in the model represents the position of a word's prosodic vector in a single 


\begin{tabular}{cccc}
\hline Dimensions & Pitch accent & Break index & Combined \\
\hline \hline chance & 53.59 & 80.41 & 38.65 \\
baseline & 84.93 & 88.34 & 75.99 \\
\hline AP11 & 86.24 & 90.71 & 78.88 \\
AP19 & 86.90 & 90.84 & 79.54 \\
L1 & 85.06 & 87.95 & 75.99 \\
L2 & 85.54 & 88.12 & 76.38 \\
L3 & 85.80 & 88.34 & 76.86 \\
L4 & 85.93 & 88.39 & 77.08 \\
L5 & 86.90 & 88.39 & 77.74 \\
L6 & 86.77 & 90.23 & 78.88 \\
L7 & 86.77 & 90.80 & 79.49 \\
L8 & $\mathbf{8 7 . 2 5}$ & $\mathbf{9 0 . 8 9}$ & $\mathbf{8 0 . 0 6}$ \\
L9 & 86.68 & 90.36 & 79.23 \\
\hline
\end{tabular}

TABLE 7.1: Pitch accent and break index classification accuracies using prosodic features and LPP dimensionality reduction.

dimension, giving a total of $r$ features per class.

\subsubsection{Results}

Classification experiments were conducted with a number of different types of language models. The baseline model used only the textual features described in Section 7.3.1. Two models were trained using the acoustic-prosodic features in the original space, incorporated using the feature function defined by Equation (7.6). Model AP11 used the 11 real-valued parameters only, while model AP19 used the full 19dimensional parameter vector. These were used in addition to the features of the baseline. In the third type of model, the set of 19 prosodic features were replaced by their lower dimensional subspace projections. The values chosen for $r$ in these LPP models ranged from 1 through to 9, corresponding to models L1 to L9, respectively. The performance of the models in this experiment were relatively insensitive to the type and variance of the prior, so all reported results have been obtained using inequality constraints (cf. Equation (4.13)) and a fixed width of $W=0.001$.

Classification accuracies for pitch accents alone, break indices alone, and the combined accent and break index labels are shown in Table 7.1. These were calculated simply as the number of correctly recognised labels as a proportion of the total number of labels. Only one joint classification was performed, and the results for the individual label types were obtained by ignoring the classification outcome of the other label. 
The first point of interest in these results is that the baseline already performed quite well. It was well above chance, and the $84.9 \%$ accuracy for pitch accents is within the neighbourhood of human inter-transcriber agreement. As expected, there is a substantial amount of useful information provided by the lexical and syntactic features alone. Incorporating the real-valued prosodic features directly further improved performance for pitch accents and break indices individually, as well as in combination. The $2.9 \%$ absolute improvement in combined label classification accuracy is significant at $p<0.01$. Appending the discrete features in model AP19 increased the accuracy by a further $0.7 \%$. Most of this gain stemmed from an improved recognition of pitch accents, supporting our claim that these features could effective capture contour information associated with accentuation. We find that using binary, integer and real-valued features in conjunction did not negatively impact the effectiveness of the model. The high levels of redundancy present in the feature set also did not appear to be a hindrance.

The performance of the LPP models, at very low dimensionalities, were close to that of the baseline. As the number of dimensions increased, though, performance increased quite sharply. At $l=5$, the pitch accent classification accuracy was greater than model AP11 and equal to AP19. Boundary classification accuracies for models L7 and L8 were comparable to, but did not significantly exceed, that of the two AP models. This would suggest that differences between accented and unaccented words are more pronounced than the distinction between boundary presence and absence, thus making their discrimination simpler to achieve with fewer features. Performance did not improve noticeably as the number of dimensions were increased beyond $l=8$, which is indicative of the high redundancy of the feature set. The feature reduction can also be seen as a method of increasing robustness, as the unimportant fluctuations are confined to the higher-order dimensions and excluded from the model.

The best results, for all types of labels, were obtained by a model using the reduced dimension features, L8. This highlights the effectiveness of the subspace projections. Performance well above the baseline could be achieved with as few as 4 dimensions. The loss of information resulting from this rather aggressive feature reduction was largely offset by the increased discrimination between the prosodic classes. Increasing the size of the projected feature vector to 8 dimensions in L8 raised the accuracy to $80 \%$, representing a $5.4 \%$ relative improvement over the baseline. The fact that this model performed better than AP19 with fewer than half the number of prosodic features demonstrates the effectiveness of the LPP in emphasising the separability between classes. The greatly reduced number of dimensions also 


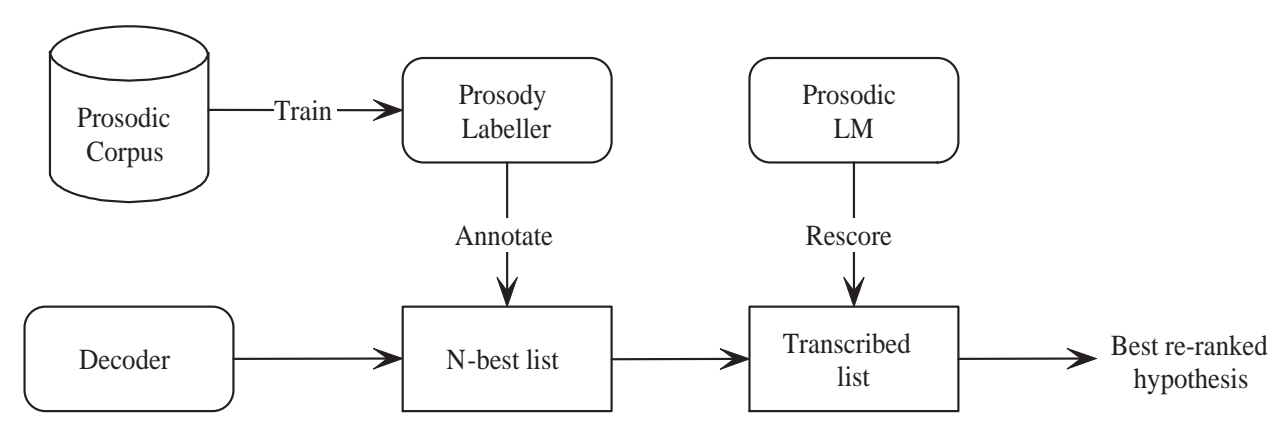

Figure 7.1: A language model rescoring system using intermediate labelling. The labeller is used to annotate the $N$-best output of a speech recogniser. These transcribed lists are then rescored by a language model that has previously been trained on the prosodic label information.

contributes to superior performance through its positive effect on distance metrics, as well as reducing computational cost.

\subsubsection{Language modelling results}

Given the reasonably good performance of this automatic prosody transcription system, it is worthwhile to attempt to apply it to the language modelling task. Assuming that a prosodically transcribed data set is available, a language can be trained on ToBI features using the process described in Chapter 5. A second model for classifying the prosodic events can also be trained on the same data. This prosodic classifier can then be used to transcribe the first-pass recogniser output of the test data, thereby allowing the original language models to be used for rescoring. This process is illustrated in Figure 7.1.

To investigate whether or not the prosody classification accuracies of the previous models are sufficient for the purposes of language modelling, we have taken the best performing of our prosodic classifiers, which used the 8-dimensional LPP, and automatically transcribed the $\mathrm{N}$-best list generated by the recogniser in conjunction with the trigram. The language model LM-ABS, was then used to rescore this list.

Surprisingly, the $77.51 \%$ accuracy achieved by this approach is identical to the result obtained using manual transcriptions. Despite the errors introduced by the automatic labelling process, the overall language model performance was largely unaltered. There are two reasons why this may be the case. The first is that the automatic labelling accuracies are almost as good as the manual transcriptions. We believe this to be the less likely explanation, though, as the $90 \%$ accuracies achieved for break index classification are still below the level of human inter-transcriber 
agreement. It is also unreasonable to claim that break index features do not contribute to the language model, as this is contrary to the results presented in Chapter 5. Instead, the lack of any substantial performance degradation is likely due to the nature of misclassifications. Content words dominate phrase-final positions in the utterances, and these are less prone to recognition errors than function words. Mislabelling a phrase-final word as not having a boundary, then, would have minimal impact on the overall recognition rate. If the recogniser is sufficiently confident in its hypothesis, the small discouragement given by the incorrect break index feature is unlikely to alter the final decision which is based on a weighted combination of all active features.

Another possible factor contributing to this performance is the absence of perceptual measures in the prosody classification process. As a result, any events which are not demarcated acoustically are eliminated. This has benefits for statistical models and the resulting transcriptions, while having less agreement with human transcriptions, are highly consistent. This is a particularly important factor which can help to offset errors introduced by incorrect classifications.

\subsection{Direct Modelling}

\subsubsection{Motivation}

In the previous section, we used automatically extracted prosodic information in a maximum entropy model to perform labelling of our data. This approach can be used to transcribe hypotheses, thus allowing the ToBI-style features to be used in the testing process. This is a straightforward approach, and its validity was supported experimentally, but it may still not be the best solution for a number of reasons.

Firstly, the automatic detection of prosodic events is far from perfect. This is supported by our results, and the $80 \%$ accuracy achieved in the 4 -way classification still leaves plenty of room for improvement. While the overall language modelling performance using the automatically transcribed $N$-best list was comparable to that of using the manual transcriptions, there is undoubtedly some beneficial information being lost by misclassifications.

Secondly, one of the potential limitations of the models identified in Chapter 5 was their inability to differentiate between alternate, prosodically consistent hypotheses. This occurred as a result of the lack of discrimination provided by the simple binary prosodic classes. Detecting these classes from the speech signal, and subsequently modelling sequences of them, introduces a quantisation error that has 
to be traded off against accuracy of prosodic event recognition. This issue can be

addressed, though, through the use of posterior probabilities [168] instead of hard boundaries.

The cascaded system also requires a second model, separate to the language model, to be developed. While training the prosodic classifier is relatively fast due to the small number of classes, this still represents an additional step in what is already a rather lengthy process. Correct transcriptions are also required for training both models. This is not entirely a disadvantage, though, as the prosodic classification component has significant re-use potential and can be trained independently of the language model.

Finally, as far as the model is concerned, the phonological representation may well be unnecessary. A statistical model is able to directly map features to the desired target classes without the need for an intermediate step. Doing this has the additional advantage of allowing the model to capture relationships which may be lost by the simplification into phonological classes. Therefore, there are benefits to using the prosodic information directly.

\subsubsection{Word clustering in prosody space}

One way in which prosodic features can be used directly in the language model is to incorporate them as per Equation (7.6). This was feasible for the prosodic classifier as each dimension of the prosodic vector introduced only 4 features into the maximum entropy model (1 per target class). Our language model, on the other hand, has approximately 4000 classes, and adding the prosodic features directly, even after dimensionality reduction, greatly increases the parameter count. Because these features are not binary, they are also active for every training sample, resulting in a very expensive parameter estimation stage.

An alternative, less costly, solution is to use a single feature per class representing the closeness of the target given the prosodic context. This can be a probability distribution, such as that used by Sandrini et al. [161], although it is not strictly necessary. We have explained earlier in this chapter that this approach results in some loss of information by not allowing the maximum entropy model to perform feature combination; however, we consider the trade-off of the reduced feature count to be worthwhile in this situation.

Recall that the construction of the similarity matrix $S$ used by the LPP transformation made use of cosine similarities to measure the closeness of points. The cosine similarity therefore provides a natural metric for comparisons in the projected 


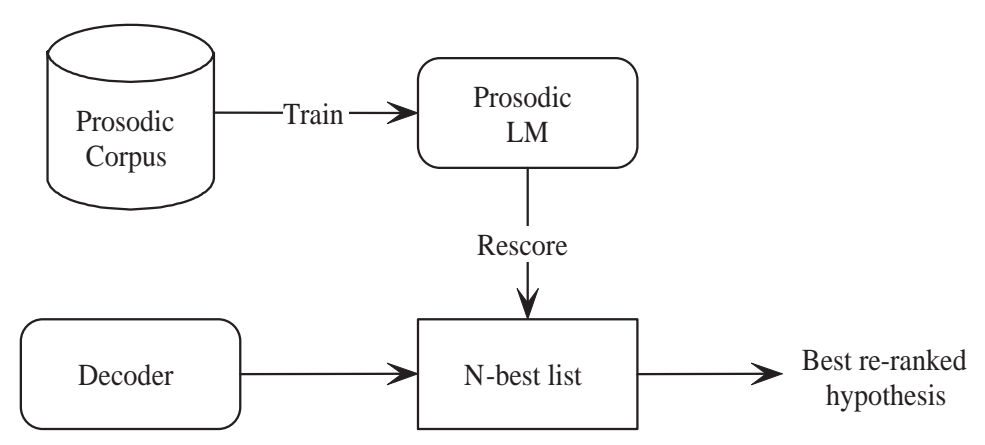

Figure 7.2: A language model rescoring system using direct prosodic features. Model features are based on the extracted prosodic parameters, thus forgoing the need for an intermediate labelling stage.

subspace. We have chosen to use these directly as model features, rather than attempt to derive a secondary probability model. This procedure gives the system architecture illustrated in Figure 7.2. For any word $w_{i}$, the set of prosodic feature vectors for all occurrences of that word in the training set can be considered to form a cluster in the prosody space. The locality of these clusters are preserved by LPP, so centroids in the subspace, $\boldsymbol{\mu}_{i}$, can be obtained for each word by taking the means of the projected vectors. A cosine similarity between a cluster centroid and an instance of a prosody vector can then be used as model features. Formally, the features we use are defined as:

$$
f_{c}(h, w)=\left\{\begin{aligned}
\operatorname{cosim}\left(\mathbf{y}_{i}, \boldsymbol{\mu}_{i}\right) & : \text { if } w=w_{i} \\
0 & : \text { otherwise }
\end{aligned}\right.
$$

where $\mathbf{y}_{i}$ is the feature vector of word $w_{i}$.

It may seem unusual to attempt to represent words in a prosody space since the same word can have multiple prosodic patterns; however, we offer the following justifications for doing so:

- Not all of the prosodic features we have used are entirely independent of word identity. In particular, features such as raw duration and some energy features depend on the underlying phoneme sequence. As a result, variations in the prosodic realisation of any given word is likely to move its position through only a subspace of the entire space spanned by the basis vectors. A similarity metric between a representative prosodic vector and an incorrectly hypothesised word can still yield a meaningful result.

- As an extension to the previous point, LPP optimises a criterion based on local 


\begin{tabular}{cc|cc}
\hline$w_{i}$ & $w_{j}$ & $g\left(w_{i}, w_{i}\right)$ & $g\left(w_{i}, w_{j}\right)$ \\
\hline \hline $\mathrm{A}$ & THE & 0.75 & 0.66 \\
$\mathrm{~A}$ & BUT & 0.75 & 0.40 \\
TO & DO & 0.71 & 0.33 \\
IN & THAN & 0.68 & 0.55 \\
IT & THAT & 0.56 & 0.33 \\
\hline
\end{tabular}

TABLE 7.2: Cosine similarities between words and cluster centroids. These particular words are easily misrecognised, leading to substitution errors.

similarities of points in the feature space, and not prosody explicitly. To the extent that word-specific information is contained in the features, separation between those words will also be improved by the projection.

- Content words, which can take on the greatest amounts of prosodic variations, are well modelled by the acoustic models. Conversely, the low acoustic salience of function words tend to make them more readily misclassified. These are less likely to exhibit high levels of prosodic variation (e.g. they are often unaccented, and rarely occur immediately before a phrase boundary) and therefore exhibit less scatter in the prosody space. As a result, a similarity metric calculated in this space can be quite accurate and is a good complement to the acoustic modelling. This should result in improved recognition rates through fewer substitution errors.

To further emphasise the third point, we examine the original $N$-best list for some of the most frequently misclassified words. These are predominantly function words, and their misclassification typically results in substitution errors in the recogniser. For each occurrence of these words in the training set, we calculate the cosine similarities between its subspace projected vector, $\mathbf{y}_{i}$, and the centroids, $\mathbf{c}$, of both the correct and incorrect word clusters. The average across all $n$ instances of word $w_{i}$ can be used as an indicator of how much separation there is between the various clusters. This is calculated as:

$$
g\left(w_{i}, w_{j}\right)=\frac{1}{n} \sum_{i=1}^{n} \operatorname{cosim}\left(\mathbf{y}_{i}, \mathbf{c}_{j}\right)
$$

The results for a selection of these words are presented in Table 7.2. Inspecting Figure $7.3 \mathrm{~s}$ plot of the cluster centroids, projected onto 3 dimensions, shows a reasonable amount of separation (the words to and $d o$ have been omitted for clarity). This is also conveyed by the average similarity scores, which are higher for the 


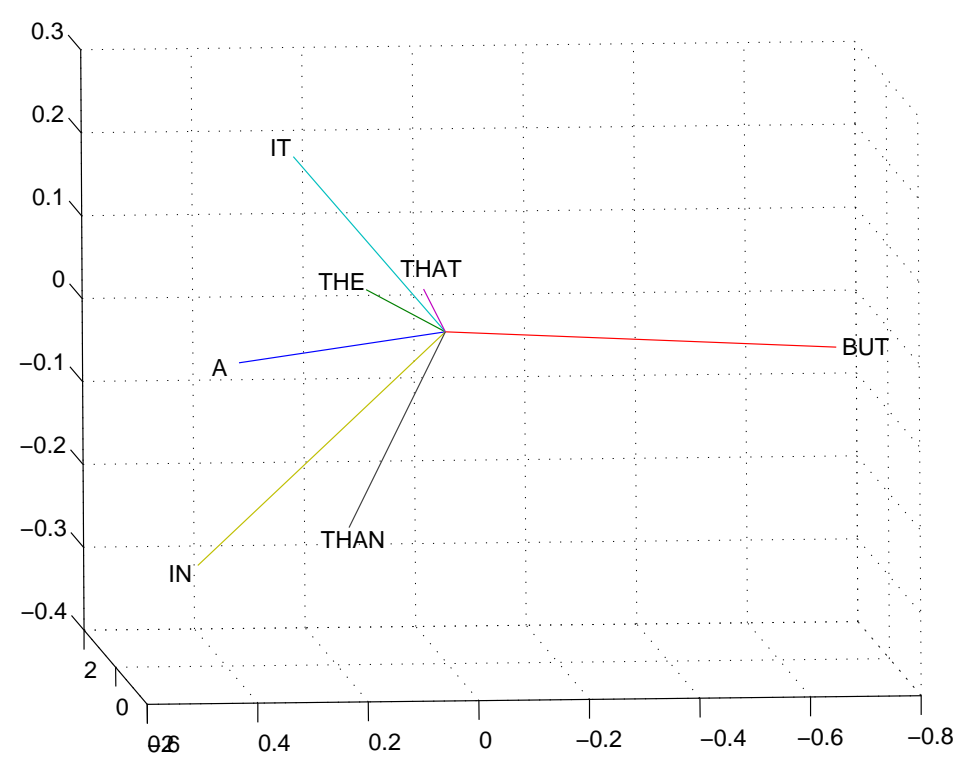

Figure 7.3: Projections of word cluster centroids onto a 3-dimensional LPP space.

within-class case than for the between-class case. All of these words, with the exception of but, lie in the positive half of the first axis. This to be expected, as they share many similarities in their usage. However, there is still sufficient distinction between the words in the remaining dimensions for the cosine similarity measure to be meaningful. Also, the actual similarity values are calculated using more dimensions than the 3 displayed in the figure, so there is in fact slightly more separability than what is depicted.

\subsubsection{Modelling prosodic history}

The cosine similarity features give an indication of how closely the prosodic pattern matches what is expected of the hypothesised word. However, it is based solely on the prosodic information extracted from the region of the word, and does not take into consideration any history of the prosody. Our experiments in Chapter 5 have indicated that modelling the prosodic pattern of at least one prior word can be beneficial, so we devise additional features to incorporate this knowledge.

To capture an aspect of the dynamics of the prosodic patterns, we propose to model the changes in the feature vectors in a way similar to the delta features used in acoustic models. At every position, we calculate the difference between the feature vectors of the current and previous words. This delta vector therefore contains information on how each dimension of the prosodic properties changes from word 
to word. It is then appended onto the original feature vector, and these extended vectors are used to calculate an LPP projection. Given the new transformation, word cluster cosine similarities can again be formulated. The precise steps are detailed below:

- For word $w_{i}$, calculate its prosodic feature vector $\mathbf{y}_{i}$.

- Calculate the difference vector as $\Delta_{i}=\mathbf{y}_{i}-\mathbf{y}_{i-1}$.

- Obtain the full feature vector, $\mathbf{y}_{i}^{\prime}$ by appending $\Delta_{i}$ to $\mathbf{y}_{i}$.

- Calculate the LPP transformation matrix based on the $\left\{\mathbf{y}_{i}^{\prime}\right\}$ of the training samples.

- Using projected word cluster means, $\boldsymbol{\mu}_{i}^{\prime}$, derive the model features as:

$$
f_{d}(h, w)=\left\{\begin{aligned}
\operatorname{cosim}\left(\mathbf{y}_{i}^{\prime}, \boldsymbol{\mu}_{i}^{\prime}\right) & : \text { if } w=w_{i} \\
0 & : \text { otherwise }
\end{aligned}\right.
$$

The resulting features, which can be used in place of those described by Equation (7.7), measure the similarity between a given sample and its expected prosodic realisation based on both its current prosodic parameters as well as its change over time. Incorporating this additional dynamic information should help to capture some important aspects of the prosodic pattern. The increase in computation is also negligible, as no more features are being added to the model. However, it is possible that this could result in a fragmentation of the word clusters in the prosody space due to the additional variation that is being modelled. This, combined with the potential need for an increase in the number of dimensions required to accurately represent the extra information, may lead to a loss of accuracy in the cosine similarity function. Its effects will be tested experimentally in the next section, where we present results for both types of features.

\subsubsection{Results}

Maximum entropy models were trained using the standard $n$-gram features (Equation (5.1)), POS features (Equation (5.4)), and the two types of prosodic features described by Equation (7.7) and Equation (7.9). LM-Cd models used the cosine similarity features based on the original feature vectors, and LM-Dd models used the feature vectors extended with first order information, where $d$ indicates the number of dimensions of the LPP subspace. All of these models also used the $n$ gram and POS features. The LM-C and LM-D models therefore differ from LM-S 


\begin{tabular}{cccc}
\hline Model & Perplexity & Accuracy & $\mathrm{p}$ \\
\hline \hline Baseline & 86.46 & 76.21 & - \\
LM-S & 71.54 & 77.06 & 0.05 \\
LM-ABS & 71.47 & 77.51 & 0.01 \\
\hline LM-C1 & 71.89 & 77.74 & 0.01 \\
LM-C2 & 68.52 & 77.80 & 0.01 \\
LM-C3 & 67.97 & 77.80 & 0.01 \\
LM-C4 & 67.33 & 77.74 & 0.01 \\
LM-C5 & 66.61 & 77.91 & 0.01 \\
LM-C6 & $\mathbf{6 6 . 4 2}$ & 77.85 & 0.01 \\
LM-C7 & 66.82 & 77.85 & 0.01 \\
LM-C8 & 66.72 & 77.85 & 0.01 \\
LM-C9 & 66.79 & 77.85 & 0.01 \\
\hline LM-D1 & 73.66 & 77.63 & 0.01 \\
LM-D2 & 70.45 & 77.80 & 0.01 \\
LM-D3 & 69.17 & 77.68 & 0.01 \\
LM-D4 & 68.49 & 77.97 & 0.001 \\
LM-D5 & 68.43 & 77.97 & 0.001 \\
LM-D6 & 68.38 & 77.91 & 0.01 \\
LM-D7 & $\mathbf{6 8 . 3 7}$ & 78.02 & 0.001 \\
LM-D8 & 68.76 & $\mathbf{7 8 . 1 4}$ & 0.001 \\
LM-D9 & 69.03 & 78.08 & 0.001 \\
\hline
\end{tabular}

TABLE 7.3: Perplexities and word recognition accuracies of models using cosine similarity features.

only by the addition of the raw prosodic features. The number of basis vectors used in the subspace projection was varied from 1 to 9 inclusive, and a cutoff of 3 was used when forming the word clusters to eliminate potentially unreliable centroids. Consequently, no cosine similarity features were defined for rarely occurring words. Inequality constraints were again used for smoothing, using a fixed width of $W=0.2$.

The perplexity and word recognition results on our language modelling task are shown in Table 7.3. For ease of comparison, the baseline, POS-only (LM-S) and best performing ToBI model (LM-ABS) results obtained in Chapter 5 are listed again here.

Both types of features, in conjunction with the POS features, led to improvements in perplexity and word accuracy. LM-D8, the model with the highest accuracy, had a $20.5 \%$ relative perplexity improvement, and a $1.93 \%$ absolute improvement in accuracy over the baseline. The word accuracy gains were significant at the level $p<0.001$. We previously posited that the inclusion of the cosine similarity 
features would aid recognition by reducing occurrences of substitution errors, and this is supported by the experimental results. In fact, the gains of LM-C and LM-D over LM-S are due almost entirely to corrections of this nature, as can be seen in the summary of error types (Table 7.4). Examination of the resulting transcriptions confirms that this approach improves modelling of function words.

Given that these features are tailored to improving the recognition of only a small subset of words, the gains may seem disproportionately large. However, the benefits of this improved modelling extend beyond simply correcting misrecognitions of function words. It is also likely that subsequent corrections will arise as a consequence of the altered state of the language model. An example of this is demonstrated in the following transcription:

$\begin{array}{cccc}\text { reference: } & \text { in three years act up } & \text { has } & \text { grown } \\ \text { trigram: } & \text { and three years act up } & \text { his } & \text { ground } \\ \text { LM-D: } & \text { and three years act up } & \text { has } & \text { grown }\end{array}$

Here, the correction of the word has can be attributed to the improved modelling by the prosodic features. A second correction by LM-D8 in the immediately following word, ground, though, is not necessarily due to the same cause. Consider the state of a bigram language model at the point where his has just been recognised. If has has been misrecognised as his, then the probabilities associated with the two best options for the subsequent word are $\mathrm{p}$ (his ground) and $\mathrm{p}$ (his grown). Neither one of these is explicitly defined in the language model, so no one hypothesis is particularly favoured over the other. Therefore, the language model only provides a minor contribution to their selection. However, when has is correctly recognised, the probabilities for the next word are changed to $\mathrm{p}$ (has ground) and $\mathrm{p}$ (has grown). The sequence has grown is a more reasonable hypothesis based on the observed training data, thus it is assigned a high probability by the bigram model. This, in turn, provides greater weight to this particular hypothesis and allows the superior transcription to be selected. This correction can therefore occur even if we make the assumption that the prosodic information does not directly influence the choice of the second word.

The difference between the two best models of each type, LM-C5 and LM-D8, with respect to the types of types of recognition errors made, is that LM-D8 produced slightly fewer insertions. This indicates that the addition of the dynamic features aids not by improving the word similarity metric, but by better modelling context and transitions between words. The extra information present in the larger feature 


\begin{tabular}{cccc}
\hline Model & Deletions & Substitutions & Insertions \\
\hline \hline baseline & 56 & 308 & 55 \\
LM-S & 52 & 294 & 56 \\
LM-C5 & 50 & 282 & 57 \\
LM-D8 & 50 & 281 & 54 \\
\hline
\end{tabular}

TABLE 7.4: Pitch accent and break index classification accuracies using prosodic features and LPP dimensionality reduction.

vectors does require more detailed modelling, as indicated by the higher optimal dimensionality of the LM-D model. This is not a significant computational burden, however, as it only affects the calculation of the cosine similarity metric.

Interestingly, the LM-C models had generally lower perplexities than the LMD models, yet failed to outperform them in the word recognition tests. While the differences are very small, the discrepancy appears to be quite consistent. We believe the cause of this to be the difference in nature of the two tasks. Perplexity measures the probability assigned to a known word sequence by a given model, while $N$-best rescoring involves a comparison between competing hypotheses. The addition of the delta features used by LM-D leads to an increase in scatter of the word clusters. The loss of precision of the cluster centroids would, in turn, lead to generally lower similarities, even for a correctly hypothesised word. Probabilities given by the LMD models would therefore tend to be lower, resulting in slightly higher perplexities. The lower probabilities apply similarly to all hypotheses, though, and so long as the increase in separation between easily confusable clusters outweighs the loss of precision, LM-D can still perform well if used as a re-ranking model.

\subsection{Noise Robustness}

One drawback of introducing a prosodic component into the language model, which is typically derived solely from textual data, is that the model becomes dependent on the quality of the acoustic signal. In practical tasks, speech acquisition conditions are rarely ideal. If the prosodic contours cannot be reliably estimated from the noisy source, then their inclusion may be of no benefit, or worse, be detrimental to overall performance.

Prosodic information is often considered to be robust to noise but this can sometimes be difficult to test. One reason for this is the lack of well-defined evaluation

criteria. While it is certainly possible to quantitatively measure, for example, a pitch contour's deviation from some reference, this is not necessarily a useful indica- 


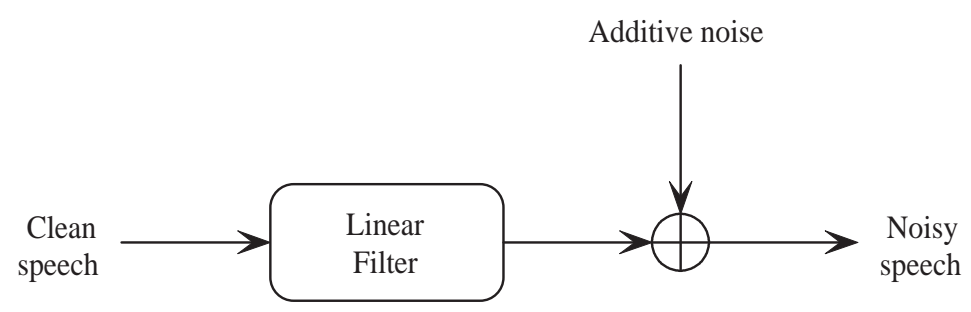

FiguRE 7.4: Modelling of two types of noise sources in the speech signal.

tor of the robustness of features derived from the contour. The wide variety of uses for prosodic information, as well as the numerous different realisations of prosodic features means any evaluation will be dependent on the intended application. In this section, we examine the effects of several types of noise on our specific task and implementation of prosodic features.

\subsubsection{Sources of noise}

There are two main types of effects which can lead to a degradation of the speech signal. One is the presence of other acoustic sources, such as vehicle traffic or speech from other speakers. Noise from these sources are typically considered to be additive and uncorrelated with the speech signal of interest. This type of noise is further classed as being either stationary (time invariant) or non-stationary. White and pink noise are examples of stationary noise. White noise is characterised by a flat power spectrum, while the spectrum of pink noise is inversely proportional to frequency. In practice, though, most noise is of the non-stationary type.

The other source of degradation is channel distortion. This may be the result of the properties of the recording equipment or transmission channel, such as a telephone line. These result in a convolutional distortion of the speech signal, and are modelled as a linear filter. Reverberation effects caused by echoic environments are another example of convolutional noise. There is also some additive noise due to reverberation, as energy from reflected speech is combined with the actual signal. The noise distortion process for the two forms of noise are illustrated in Figure 7.4.

\subsubsection{Results}

For investigating additive noise, the test set samples were mixed with one of white, pink, babble or factory ${ }^{1}$ noise samples obtained from the NOISEX-92 database [169].

\footnotetext{
${ }^{1}$ factory1.wav
} 


\begin{tabular}{c|cccc}
\hline \multirow{2}{*}{ noise } & \multicolumn{4}{|c}{ SNR (dB) } \\
& 20 & 10 & 5 & 0 \\
\hline white & 78.14 & 78.14 & 78.14 & 78.14 \\
pink & 78.14 & 78.14 & 78.14 & 77.97 \\
babble & 78.14 & 77.97 & 77.91 & 77.85 \\
factory & 78.14 & 78.08 & 78.08 & 77.91 \\
\hline
\end{tabular}

TABLE 7.5: Word accuracies of LM-D8 when using prosodic features extracted from data corrupted by additive noise.

This was performed using the Filtering and Noise Adding Tool [170], for SNRs ${ }^{2}$ ranging from $20 \mathrm{~dB}$ down to $0 \mathrm{~dB}$. Because we wish to isolate the prosodic component, MFCC parameters used by the acoustic models were calculated from clean speech in both training and testing. Only the F0 and RMS energy extraction procedures were performed on the corrupted speech in the testing phase. Dimensionality reduction during testing was performed by transforming the noisy prosodic feature vector with a projection matrix calculated from clean training data. Therefore, the following results are not indicative of the performance of the complete system under noisy conditions. All results are reported using the language model LM-D8.

The results in Table 7.5 demonstrate that these features are very robust to additive stationary noise. No degradation in performance was observed for white noise, and there was only a minor decrease in accuracy at 0 and $5 \mathrm{~dB}$ for pink noise. The prosodic features we have used are derived from the automatically extracted pitch contour, so their behaviour in noisy conditions will be closely related. Because both white and pink noise do not have any periodicity, their presence has minimal effect on the pitch tracking algorithm. As a result, the prosodic features derived from this output are similarly unaffected. In the presence of babble and factory noise, the performance degradation was more evident. Babble noise, in particular, can interfere with the pitch extraction at low SNRs. Segments of F0 contours, all extracted from the same region, but for different types of noise, are illustrated in Figure 7.5. Babble and factory noise alter the contour to a greater extent than the two stationary noises; however, all contours exhibited noticeable deviations from the clean contour. The fact that performance under white and pink noise remained high, then, suggests that the following stages of feature processing (i.e. smoothing, subspace projection and clustering) are also robust to this type of noise.

The models retain good performance at fairly low SNRs, but at SNRs below $0 \mathrm{~dB}$, the pitch tracker would often fail completely. Without a pitch contour, no

\footnotetext{
${ }^{2}$ SNRs were calculated after applying an A-weight filter
} 


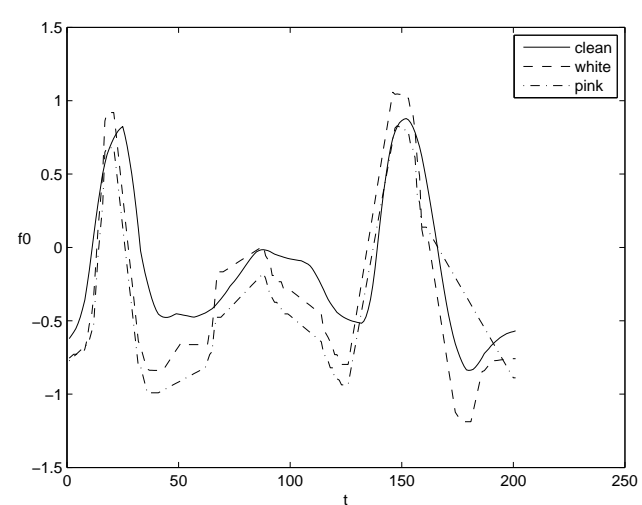

(a) Stationary noise

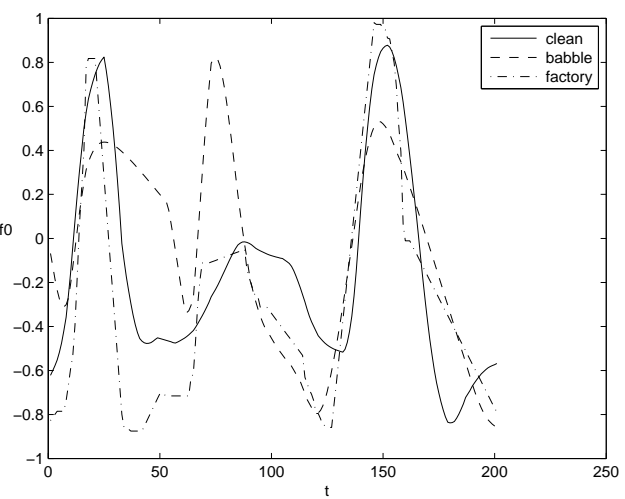

(b) Non-stationary noise

FigURE 7.5: F0 contours extracted from signals corrupted with additive noise at a SNR of $0 \mathrm{~dB}$.

meaningful features can be derived. Therefore, these prosodic features are of no use in extremely noisy conditions, but are highly robust up to this threshold.

Similar tests were conducted for reverberant noise. The Roomsim package [171] was used to simulate the impulse response of an enclosed rectangular room with dimensions $8 \mathrm{~m} \times 8 \mathrm{~m} \times 2.5 \mathrm{~m}$. The receiver was positioned at $2 \mathrm{~m} \times 4 \mathrm{~m} \times 1.1$ $\mathrm{m}$, and the source was located $1 \mathrm{~m}$ away at the same elevation, at $3 \mathrm{~m} \mathrm{x} 4 \mathrm{mx}$ $1.1 \mathrm{~m}$. The level of reverberation can be quantified in terms of reverberation time $\left(\mathrm{RT}_{60}\right)$, which specifies the length of time required for a sound to decay by $60 \mathrm{~dB}$. Five impulse responses were generated by adjusting the surface absorptions of the walls such that their $R_{60} \mathrm{~s}$ ranged from $0.1 \mathrm{~s}$ to $0.5 \mathrm{~s}$, in $0.1 \mathrm{~s}$ increments. The dimensions of the room remained the same in all cases. The results in Table 7.5.2 were obtained by performing prosodic feature extraction on the convolution of the clean speech signal with one of the simulated impulse responses.

Once again, the prosodic features exhibited a high degree of robustness in this type of noise. Examination of the extracted pitch contours (Figure 7.6) shows that F0 is largely invariant to channel distortions. Reverberation results in the presence of an attenuated form of the signal occurring at a slight delay, and causes sampled feature values to be merged across the time domain. While this poses a problem for short-time spectral features, it is not a major issue for the suprasegmental prosodic features, which are calculated over longer time periods. In fact, the effect of reverberation is not entirely dissimilar to the median smoothing applied to the F0 and 


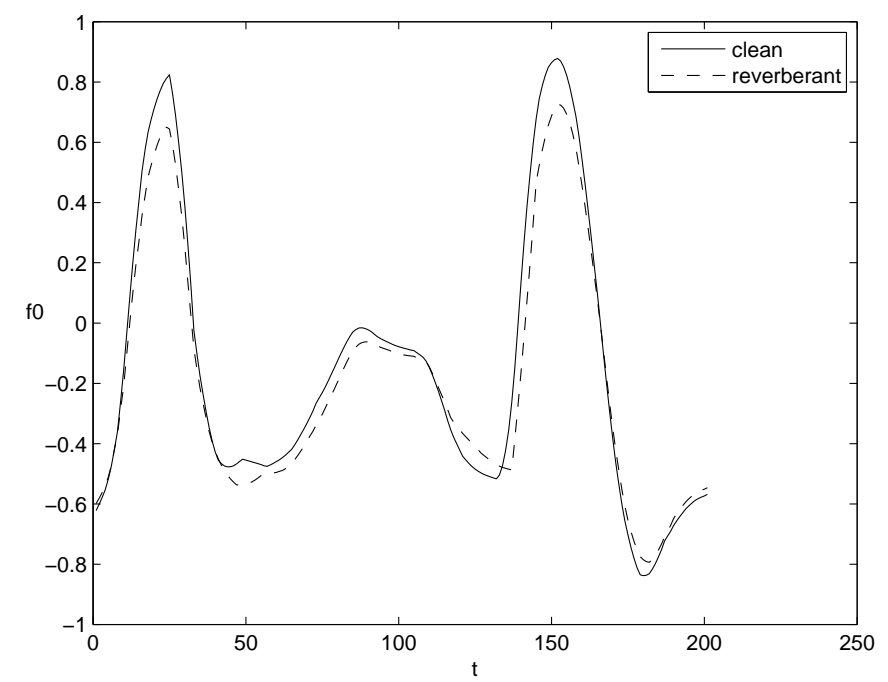

FiguRE 7.6: F0 contour extracted from a signal in the presence of reverberation with a $\mathrm{RT}_{60}$ of $0.5 \mathrm{~s}$.

\begin{tabular}{c|ccccc}
\hline \multirow{2}{*}{ noise } & \multicolumn{5}{|c}{$\mathrm{RT}_{60}$} \\
& 0.1 & 0.2 & 0.3 & 0.4 & 0.5 \\
\hline reverberant & 78.14 & 78.14 & 78.14 & 78.08 & 77.91 \\
\hline
\end{tabular}

TABLE 7.6: Word accuracies of LM-D8 when using prosodic features extracted from data corrupted by reverberant noise.

energy contours in the post-processing stage.

Another explanation for the high level of robustness, particularly against reverberant noise, is that the feature vector contains duration information derived from the original $N$-best list. If this list were to be regenerated under noisy conditions, the differences to the hypothesised words and their time alignments would impact upon the duration features. However, it should also be noted that the transformation applied to the feature vector prevents the resulting model from attributing undue weight to the durational information alone. Therefore, we believe that these results do indicate that F0 and energy features are quite robust to noise.

\subsection{Conclusion}

In this chapter, we have described two methods in which automatically extracted features may be used for language modelling. Irrespective of which approach is taken, it is useful to first perform dimensionality reduction on the original prosodic 
feature vectors. Doing so can greatly reduce the number of candidate features. For a model that is computationally expensive to train, this is of significant benefit. Reductions in computation are not the sole reason, though. Dimensionality reduction can serve to minimise the effects of noise in the data, and techniques such as LPP can emphasis the separation of classes in the lower dimensional space. When modelled in an appropriate manner, the combination of these effects can lead to performance in excess of what can be obtained using the original, high-dimensional feature vectors.

One potential use for the automatically extracted prosodic features is prosody labelling. Our maximum entropy model, trained to classify four combinations of accentuation and boundaries, benefited significantly from the subspace projected features. This model can be used to annotate the output of a speech recogniser, enabling language models augmented with ToBI features to be applied. Experiments conducted with this approach gave an improvement of $1.3 \%$ over the trigram model, which was equal to the gain achieved by the models in Chapter 5 using manually transcribed data.

We also presented a technique for using the prosodic information directly in the language model. Such an approach has the benefit of avoiding the intermediate transcription step. In fact, it does not require any knowledge of phonological classes at all. This is important for statistical modelling, as it opens up the possibility of using much larger, unannotated corpora. The large number of classes in the language model, as well as the weak relationship between prosody and word identity, means care needs to be taken when formulating model features. We proposed an approach based on cosine similarities, which is the same metric used by the subspace projection algorithm. We also derived a compact set of features to represent the short-term prosodic history. Language models trained on these two types of features performed well, and actually exceeded the accuracies achieved by using ToBI features $(1.9 \%$ vs $1.3 \%)$.

Lastly, we conducted some experiments to test the robustness of these prosodic features in various noisy conditions. We find that they are very robust to reverberant noise. Only minimal performance degradations were observed, up to and including an $\mathrm{RT}_{60}$ of $0.5 \mathrm{~s}$. This is due to the accurate pitch tracking in these conditions, which is largely invariant to convolutional distortion. Robustness to stationary additive noise was also high. Performance did not suffer under white noise until the SNR dropped $0 \mathrm{~dB}$, and $5 \mathrm{~dB}$ under pink noise. Although the pitch extraction was influenced by the noise, the subsequent processing stages were able to compensate for the minor deviations. In the presence of babble and factory noise, the prosodic 
features were slightly less robust at low SNRs. The major issue with additive noise is the tendency for the pitch tracking algorithm to fail to identify a meaningful contour. Up until this point, though, the features demonstrated good robustness in general. 


\section{Chapter 8}

\section{Conclusion}

This chapter will briefly summarise the main findings of this research, and highlight its contributions. It will also present some further research directions and extensions to this work.

\subsection{Summary}

Research into the use of prosody for ASR and other speech processing systems has gained momentum in recent years, and many approaches for modelling prosody have been developed. With regard to ASR applications, the focus has primarily been on augmenting phoneme models with prosodic parameters. In our work, we have considered prosody as a higher level phenomenon, and proposed an approach for incorporating this information into a language model.

In Chapter 5, we investigated some possible uses of perceptually transcribed prosodic information for enhancing language models. An analysis of the mutual information between words and their POS and ToBI labels indicated that there were strong correlations between the three knowledge sources. Syntax and prosody are closely related; however, the POS and ToBI labels were not redundant, and in fact provided complementary information to each other. ToBI labels also did not demonstrate a rapid decline in mutual information with word identities as the distance from the word increased.

We modelled sequences of POS, pitch accent and break index labels by formulating them as features for a maximum entropy language model. These models provide an effective framework for combining information from multiple, potentially disparate knowledge sources, and this was demonstrated by the gains obtained through using the additional features. Using the three types of features, in conjunction with word $n$-gram features, led to a $19 \%$ relative improvement in perplexity over the base- 
line trigram model. The maximum entropy model also performed well in $N$-best list reranking tests, increasing the recognition accuracy by a relative $1.7 \%$.

Our experiments also highlighted some limitations to the use of ToBI transcriptions in this manner. Firstly, we found no benefit to modelling contexts longer than 3 words. Both perplexity and recognition accuracies were degraded by modelling longer histories. The primary reason for this, we believe, is that the labelling of the data is not sufficiently consistent to be fully exploited by the model. Attempting to gather statistics from long sequences of these labels exacerbates any inconsistencies, and as a result, the statistics will be unreliable.

The prosodic features aid the language model by penalising hypotheses that are not consistent with the observed prosodic context. However, the features themselves have very coarse granularity. In an $N$-best list, there are many similar hypotheses, and it is very likely that it will contain more than one hypothesis that is consistent with the modelled prosodic information. Therefore, the new models tend to improve the rankings of several hypotheses equally, while being unable to differentiate between individual options within that subset.

Drawing from these findings, we extended our analysis to raw acoustic-prosodic data. In the work documented in Chapter 6, we examined a number of duration, F0 and energy features for correlations to prosodic events. Because sub-word time alignment was not readily available in our task, we concentrated on the use of word level features only, and developed a parameterisation of F0 and energy contours designed specifically to encode information at this level. The analysis of the acoustic-prosodic data has contributed to the understanding of the behaviour of these parameters under differing prosodic conditions, and has also shown that our proposed features are particularly effective at characterising the contour patterns associated with accentuation.

To confirm the validity of the set of prosodic features developed in Chapter 6, we used them to train a prosody classifier in Chapter 7. The results supported our claim that the additional features we proposed were effective at capturing relevant information on accentuation. In this chapter, we also discussed the applicability of linear dimensionality reduction methods for our feature set, and explained why the properties of the LPP technique made it suitable for our task. This was confirmed experimentally in the prosody classification experiment, where the model trained on a reduced set of features outperformed an equivalent model using the full set of features. The best performing model used a feature vector with fewer than half the original number of dimensions, and achieved a $5.4 \%$ relative improvement in classification accuracy over a baseline model using lexical features. 
In Section 7.4 we explored the problem of directly using the raw prosodic parameters in a language model. Creating phonological transcriptions of data is laborious, and some prior work in this area has alleviated the burden by developing techniques for automatically annotating data with prosodic-syntactic information. In our approach, we have taken this idea further and removed the need for the intermediate transcription step altogether. We proposed a novel technique of representing the prosodic information based on the properties of the subspace projection that is both compact and effective at characterising the prosodic context of words. An examination of words commonly misclassified by the original trigram model suggested that these features, when used by a language model, provided a good complement to the acoustic models. Evaluation of models trained using these features showed that they performed well, and actually exceeded the ToBI feature models in both perplexity and recognition accuracy. As with the prosody classification experiments, a moderate dimensionality reduction gave the best results, with the best model demonstrating a $20.5 \%$ relative improvement in perplexity over the baseline, and a $2.5 \%$ relative gain in recognition rate.

Prosody is considered to be robust against noise, but this is not often tested. In the final section of this chapter, we conducted experiments to verify the robustness of our prosodic features under a variety of noisy environments. Including acousticprosodic information into the language model has the drawback of introducing a dependence on speech signal quality, and this could potentially negate benefits of using the features if they degrade rapidly with noise. Our experiments indicated that this was not a major issue, however, as the features exhibited a high degree of robustness to all types of tested conditions. The pitch contour extraction procedure was largely unaffected by the presence of convolutional distortion, and subsequently, so were our derived features. Additive noise did influence the pitch extraction, but the dimensionality reduction and further processing of the features also proved to be quite robust. Overall, only minimal performance degradation was observed, even at the relatively low $\mathrm{SNR}$ of $0 \mathrm{~dB}$.

\subsection{Future Research}

There is still much to learn about the nature of prosody, both in human-to-human communication and as it applies to automatic speech processing. With regard to our current research, though, there are a number of specific issues which are worth drawing attention to. 


\subsubsection{Alternate Corpus}

Firstly, it would be worthwhile to test our methods on a different corpus. The RNC has been purposefully designed for prosody research, and the professionally read speech gives very controlled and prosodically well-formed realisations. It is possible that the statistical analyses and modelling used here may utilise these regularities to the exclusion of other prosodic effects. In particular, spontaneous and conversational speech is significantly different in its prosodic structure, and it is not apparent how our techniques would perform on such data.

\subsubsection{Improved Prosody Modelling}

Throughout this study we have, where possible, attempted to use prosodic information directly as features in the maximum entropy models. This was primarily motivated by the belief that a direct approach can eliminate a potential source of error caused by an intermediate modelling step. The simple features have also been a useful tool in helping to understanding the behaviour and interaction of prosodic information. However, much like $n$-grams, such basic features do not capture the full extent of the relationship between the modelled variables. Our last set of experiments, too, have shown that intermediate representations of prosody can be useful, provided they are sufficiently reliable. More detailed features are certainly not problematic given the very general framework of the maximum entropy model. As an example, encouraging results have been achieved by encoding dependency grammar constraints into a maximum entropy language model [33]. Similarly, explicitly modelling prosodic structure could also lead to further gains. There is a wealth of knowledge available about many aspects of prosody and phonology, though coercing this information into a representation conducive to statistical modelling is not a trivial task.

\subsubsection{System Integration}

Tighter integration between language and acoustic models is another important issue, not only for our work, but for language modelling research in general. While the $N$-best rescoring approach we have used is helpful for developmental purposes, a complete system should make use of the prosodic knowledge at an earlier stage in order to avoid the propagation of errors. Word graphs and lattices are superior to $N$-best lists in terms of coverage, but are still generated using phoneme models and linguistically deficient $n$-grams. Managing the trade-off between early application of 
knowledge sources and computational tractability remains a difficult problem, yet it is one that needs to be addressed in order to fully exploit prosodic and syntactic information.

In this work, we have demonstrated some ways in which suprasegmental prosodic information can aid word recognition. Ultimately, though, prosody is responsible for higher level structures relating to syntax, semantics and dialogue. While these effects are not directly relevant to the automatic word recognition problem, they are an important component of human speech comprehension. Due to the close and complex relationships between the many levels of speech communication, examining each one in isolation is perhaps less meaningful than considering it as a single, integrated unit. Prosody is not necessarily the only aspect of speech which affects so many levels of the process, but the difficulties encountered in quantifying its precise functionality certainly underlines the need for a more holistic approach to developing automatic speech processing systems. 


\section{Appendix A}

\section{Penn Treebank Tag Set}

This is the set of word categories in the Penn Treebank with which the Boston University Radio Speech Corpus has been annotated.

$\begin{array}{cc}\text { CC } & \text { Coordinating conjunction } \\ \text { CD } & \text { Cardinal number } \\ \text { DT } & \text { Determiner } \\ \text { EX } & \text { Existential there } \\ \text { FW } & \text { Foreign word } \\ \text { IN } & \text { Preposition or subordinating conjunction } \\ \text { JJ } & \text { Adjective } \\ \text { JJR } & \text { Adjective, comparative } \\ \text { JJS } & \text { Adjective, superlative } \\ \text { LS } & \text { List item marker } \\ \text { MD } & \text { Modal } \\ \text { NN } & \text { Noun, singular or mass } \\ \text { NNS } & \text { Noun, plural } \\ \text { NP } & \text { Proper noun, singular } \\ \text { NPS } & \text { Proper noun, plural } \\ \text { PDT } & \text { Predeterminer } \\ \text { POS } & \text { Possessive ending } \\ \text { PP } & \text { Personal pronoun } \\ \text { PP\$ } & \text { Possessive pronoun } \\ \text { RB } & \text { Adverb } \\ \text { RBR } & \text { Adverb, comparative } \\ \text { RBS } & \text { Adverb, superlative } \\ \text { RP } & \text { Particle }\end{array}$




$\begin{array}{cc}\text { SYM } & \text { Symbol } \\ \text { TO } & \text { to } \\ \text { UH } & \text { Interjection } \\ \text { VB } & \text { Verb, base form } \\ \text { VBD } & \text { Verb, past tense } \\ \text { VBG } & \text { Verb, gerund or present participle } \\ \text { VBN } & \text { Verb, past participle } \\ \text { VBP } & \text { Verb, non-3rd person singular present } \\ \text { VBZ } & \text { Verb, 3rd person singular present } \\ \text { WDT } & \text { Wh-determiner } \\ \text { WP } & \text { Wh-pronoun } \\ \text { WP\$ } & \text { Possessive wh-pronoun } \\ \text { WRB } & \text { Wh-adverb }\end{array}$




\section{Bibliography}

[1] A. Cutler, D. Dahan, and W. van Donselaar, "Prosody in the comprehension of spoken language: A literature review," Language and Speech, vol. 40, no. 2, pp. 141-201, 1997.

[2] R. Jakobson, C. Gunnar, M. Fant, and M. Hall, Preliminaries to Speech Analysis: The Distinctive Features and their Correlates. Cambridge, USA: MIT Press, 1967.

[3] D. R. Ladd, Intonational Phonology. Cambridge, Great Britain: Cambridge University Press, 1996.

[4] D. L. Bolinger, "Intonation across languages," Universals of Human Language, vol. 2, pp. 471-524, 1978.

[5] F. Grosjean, "How long is the sentence? Prediction and prosody in the on-line processing of language," Linguistics, vol. 21, pp. 501-529, 1983.

[6] I. Lehiste, "The timing of utterances and linguistic boundaries," Journal of the Acoustical Society of America, vol. 51, no. 6B, pp. 2018-2024, 1972.

[7] A. Waibel, Prosody and Speech Recognition. San Mateo, USA: Morgan Kaufmann Publishers, 1988.

[8] R. Collier and J. 't Hart, "The role of intonation in speech perception," in Structure and Process in Speech Perception, A. Cohen and S. G. Nooteboom, Eds. New York, USA: Springer-Verlag, 1975.

[9] I. Bulyko, M. Ostendorf, and P. Price, "On the relative importance of different prosodic factors for improving speech synthesis," in Proc. International Congress of Phonetic Sciences, San Francisco, CA, USA, 1999, pp. 81-84.

[10] G. Lidstone, "Note on the general case of the Bayes-Laplace formula for inductive or a posteriori probabilities," Transactions of the Faculty of Actuaries, vol. 8, pp. 182-192, 1920. 
[11] W. Gale and K. Church, "What is wrong with adding one?" in Corpus-Based Research into Language, N. Oostdijk and P. D. Haan, Eds. Amsterdam, The Netherlands: Rodopi, 1994, pp. 189-198.

[12] I. J. Good, "The population frequencies of species and the estimation of population parameters," Biometrika, vol. 40, no. 3-4, pp. 237-264, 1953.

[13] W. A. Gale, "Good-Turing smoothing without tears," AT\&T Bell Laboratories, Tech. Rep., 2000.

[14] F. Jelinek and R. L. Mercer, "Interpolated estimation of Markov source parameters from sparse data," in Proc. Workshop on Pattern Recognition in Practice, Amsterdam, The Netherlands, 1980, pp. 381-397.

[15] L. R. Bahl, F. Jelinek, and R. L. Mercer, "A maximum likelihood approach to continuous speech recognition," IEEE Transactions on Pattern Analysis and Machine Intelligence, vol. 5, no. 2, pp. 179-190, 1983.

[16] S. M. Katz, "Estimation of probabilities from sparse data for the language model component of a speech recognizer," IEEE Transactions on Acoustics, Speech, and Signal Processing, vol. 35, no. 3, pp. 400-401, 1987.

[17] S. F. Chen and J. Goodman, "An empirical study of smoothing techniques for language modeling," Computer Speech and Language, vol. 10, pp. 359-393, 1999 .

[18] H. Ney, U. Essen, and R. Kneser, "On structuring probabilistic dependencies in stochastic language modelling," Computer Speech and Language, vol. 8, no. 1, pp. 1-38, 1994.

[19] R. Kneser and H. Ney, "Improved backing-off for m-gram language modeling," in Proc. International Conference on Acoustics, Speech, and Signal Processing, 1995, pp. 181-184.

[20] P. F. Brown, V. J. Della Pietra, P. V. deSouza, and J. C. Lai, "Class-based n-gram models of natural language," Computational Linguistics, vol. 18, no. 4, pp. 467-479, 1992.

[21] J. Gao, J. Goodman, and J. Miao, "The use of clustering techniques for Asian language modeling," Computational Linguistics and Chinese Language Processing, vol. 6, no. 1, pp. 27-60, 2001. 
[22] R. Kneser and H. Ney, "Improved clustering techniques for class-based statistical language modeling," in Proc. Eurospeech, 1993, pp. 973-976.

[23] K. Kirchhoff, J. Bilmes, S. Das, N. Duta, M. Egan, G. Ji, F. He, J. Henderson, D. Liu, M. Noamany, P. Schone, R. Schwartz, and D. Vergyri, "Novel approaches to Arabic speech recognition: Report from the 2002 John-Hopkins summer workshop," in Proc. International Conference on Acoustics, Speech, and Signal Processing, Hong Kong, China, 2003, pp. 344-347.

[24] J. Bilmes and K. Kirchhoff, "Factored language models and generalized parallel backoff," in Proc. Conference of the North American Chapter of the Association for Computational Linguistics on Human Language Technology, Edmonton, Canada, 2003, pp. 4-6.

[25] K. Kirchhoff and M. Yang, "Improved language modeling for statistical machine translation," in Proc. ACL Workshop on Building and Using Parallel Texts, 2005.

[26] K. Duh and K. Kirchhoff, "Automatic learning of language model structure," in Proc. International Conference on Computational Linguistics, Geneva, Switzerland, 2004, pp. 148-154.

[27] J. Baker, "Trainable grammars for speech recognition," in Proc. Spring Conference of the Acoustical Society of America, Boston, MA, USA, 1979, pp. 547-550.

[28] E. Charniak, "Immediate-head parsing for language models," in Proc. 39th Annual Meeting of the Association for Computational Linguistics, San Francisco, CA, USA, 2001, pp. 116-123.

[29] A. Stolcke and J. Segal, "Precise $n$-gram probabilities from stochastic contextfree grammars," in Proc. 32nd Annual Meeting of the Association for Computational Linguistics, Las Cruces, NM, USA, 1994, pp. 74-79.

[30] J. H. W. H. Lloyd-Thomas and G. J. F. Jones, "An integrated grammar/bigram language model using path scores," in Proc. International Conference on Acoustics, Speech, and Signal Processing, Detroit, MI, USA, 1995, pp. $173-176$.

[31] C. Chelba and F. Jelinek, "Exploiting syntactic structure for language modeling," in Proc. 36th Annual Meeting of the Association for Computational Linguistics, Montreal, Canada, 1998, pp. 225-231. 
[32] L. Tesniére, Élements de Syntaxe Structurale. Paris, France: Klincksieck, 1959.

[33] C. Chelba, D. Engle, F. Jelinek, V. Jimenez, S. Khudanpur, L. Mangu, H. Printz, E. Ristad, R. Rosenfeld, A. Stolcke, and D. Wu, "Structure and performance of a dependency language model," in Proc. European Conference on Speech Communication and Technology, Rhodes, Greece, 1997, pp. 2775-2778.

[34] J. Gao, J.-Y. Nie, G. Wu, and G. Cao, "Dependence language model for information retrieval," in Proc. 27th Annual International ACM SIGIR Conference on Research and Development in Information Retrieval, Sheffield, United Kingdom, 2004, pp. 170-177.

[35] L. R. Bahl, P. F. Brown, P. V. deSouza, and R. L. Mercer, "A tree-based statistical language model for natural language speech recognition," IEEE Transactions on Acoustics, Speech, and Signal Processing, vol. 37, no. 7, pp. 1001-1008, 1989.

[36] R. Rosenfeld, "A maximum entropy approach to adaptive statistical language modeling," Computer Speech and Language, vol. 10, no. 3, pp. 187-228, 1996.

[37] L. R. Rabiner, "A tutorial on hidden Markov models and selected applications in speech recognition," Proceedings of the IEEE, vol. 77, no. 2, pp. 257-286, 1989.

[38] H. Hermansky, "Perceptual linear predictive (PLP) analysis of speech," Journal of the Acoustical Society of America, vol. 87, no. 4, pp. 1738-1752, 1990.

[39] S. B. Davis and P. Mermelstein, "Comparison of parametric representations for monosyllabic word recognition in continuously spoken sentences," IEEE Transactions on Acoustics, Speech, and Signal Processing, vol. 28, no. 4, pp. 357-366, 1980.

[40] L. R. Rabiner and B. H. Juang, "An introduction to hidden Markov models," IEEE ASSP Magazine, vol. 3, no. 1, pp. 4-16, 1986.

[41] X. D. Huang and M. A. Jack, "Semi-continuous hidden Markov models in isolated word recognition," in Proc. 9th International Conference on Pattern Recognition, Rome, Italy, 1988, pp. 406-408. 
[42] L. E. Baum, T. Petrie, G. Soules, and N. Weiss, "A maximization technique occurring in the statistical analysis of probabilistic functions of Markov chains," Annals of Mathematical Statistics, vol. 41, no. 1, pp. 164-171, 1970.

[43] A. P. Dempster, N. M. Laird, and D. B. Rubin, "Maximum likelihood from incomplete data via the EM algorithm," Journal of the Royal Statistical Society, vol. 39, no. 1, pp. 1-21, 1977.

[44] N. Merhav and Y. Ephraim, "Maximum likelihood hidden Markov modeling using a dominant sequence of states," IEEE Transactions on Signal Processing, vol. 39, no. 9, pp. 2111-2114, 1991.

[45] B. T. Lowerre, "The HARPY speech recognition system," Ph.D. dissertation, Carnegie Mellon University, 1976.

[46] F. Jelinek, "A fast sequential decoding algorithm using a stack," IBM Journal of Research and Development, vol. 13, pp. 675-685, 1969.

[47] L. R. Bahl, P. S. Gopalakrishnam, D. Kanevsky, and D. Nahamoo, "Matrix fast match: A fast method for identifying a short list of candidate words for decoding," in Proc. International Conference on Acoustics, Speech, and Signal Processing, Glasgow, Scotland, 1989, pp. 81-84.

[48] Y. Chow and R. Schwartz, "The N-best algorithm: An efficient procedure for finding top N sentence hypotheses," in Proc. DARPA Workshop on Speech and Natural Language, Cape Cod, MA, USA, 1989, pp. 199-202.

[49] X. Aubert, C. Dugast, H. Ney, and V. Steinbiss, "Large vocabulary continuous speech recognition of Wall Street Journal data," in Proc. International Conference on Acoustics, Speech, and Signal Processing, Adelaide, Australia, 1994, pp. 129-132.

[50] R. A. Wagner and M. J. Fischer, "The string-to-string correction problem," Journal of the Association for Computing Machinery, vol. 21, pp. 168-173, 1974.

[51] L. Gillick and S. Cox, "Some statistical issues in the comparison of speech recognition algorithms," in Proc. International Conference on Acoustics, Speech, and Signal Processing, Glasgow, Scotland, 1989, pp. 523-535.

[52] D. S. Pallett, W. M. Fisher, and J. G. Fiscus, "Tools for the analysis of benchmark speech recognition tests," in Proc. International Conference on 
Acoustics, Speech, and Signal Processing, Albuquerque, NM, USA, 1990, pp. 97-100.

[53] S. Coren, L. M. Ward, and J. T. Enns, Sensation and Perception. New York, USA: Academic Press, 1979.

[54] D. Talkin, "A robust algorithm for pitch tracking," in Speech Coding and Synthesis, W. B. Kleijn and K. K. Paliwal, Eds. Elsevier, 1995.

[55] T. Shimamura and H. Kobayashi, "Weighted autocorrelation for pitch extraction of noisy speech," IEEE Transactions on Speech and Audio Processing, vol. 9, no. 7, pp. 727-730, 2001.

[56] H. Fletcher and W. A. Munson, "Loudness, its definition, measurement and calculation," Journal of the Acoustical Society of America, vol. 5, pp. 82-108, 1933.

[57] R. Kompe, Prosody in Speech Understanding Systems, ser. Lecture Notes in Artificial Intelligence. Berlin, Germany: Springer-Verlag, 1997.

[58] C. W. Wightman, "Automatic detection of prosodic constituents for parsing," Ph.D. dissertation, Boston University, 1992.

[59] J. P. H. van Santen, "Assignment of segmental duration in text-to-speech synthesis," Computer Speech and Language, vol. 8, no. 2, pp. 95-128, 1994.

[60] M. Liberman and A. Prince, "On stress and linguistic rhythm," Linguistic Inquiry, vol. 8, no. 2, pp. 249-336, 1977.

[61] S. Nooteboom, "The prosody of speech: Melody and rhythm," in The Handbook of Phonetic Sciences, W. J. Hardcastle and J. Laver, Eds. Blackwell, 1997, pp. 640-673.

[62] P. Lieberman, "Some acoustic correlates of word stress in American English," The Journal of the Acoustical Society of America, vol. 32, no. 4, pp. 451-454, 1960 .

[63] G. Kochanski, E. Grabe, J. Coleman, and B. Rosner, "Loudness predicts prominence: Fundamental frequency lends little," The Journal of the Acoustical Society of America, vol. 118, no. 2, pp. 1038-1054, 2005.

[64] J. 't Hart, R. Collier, and A. Cohen, A Perceptual Study of Intonation. Cambridge, Great Britain: Cambridge University Press, 1990. 
[65] L. B. Leonard, "The role of intonation in the recall of various linguistic stimuli," Language and Speech, vol. 16, no. 4, pp. 327-335, 1973.

[66] G. M. Robinson, "Rhythmic organization in speech processing," Journal of Experimental Psychology: Human Perception and Performance, vol. 3, pp. 83-91, 1977.

[67] R. K. Bansal, The Intelligibility of Indian English. Central Institute of English and Foreign Languages, 1976.

[68] J. G. Martin, "Temporal word spacing and the perception of ordinary, anomalous, and scrambled strings," Journal of Verbal Learning and Verbal Behavior, vol. 7 , pp. $154-157,1968$.

[69] C. J. Darwin, "On the dynamic use of prosody in speech perception," in Structure and Process in Speech Perception, A. Cohen and S. G. Nooteboom, Eds. New York, USA: Springer-Verlag, 1975.

[70] A. Treisman, "Contextual cues in selective listening," Quarterly Journal of Experimental Psychology, vol. 12, pp. 242-248, 1960.

[71] R. H. Meltzer, J. G. Martin, C. B. Mills, D. L. Imhoff, and D. Zohar, "Reaction time to temporally-displaced phoneme targets in continuous speech," Journal of Experimental Psychology: Human Perception and Performance, vol. 2, no. 2, pp. 277-290, 1976.

[72] F. Ferreira, M. D. Anes, and M. D. Horine, "Exploring the use of prosody during language comprehension using the auditory moving window technique," Journal of Psycholinguistic Research, vol. 25, no. 2, pp. 273-290, 1996.

[73] B. A. Blesser, "Perception of spectrally rotated speech," Ph.D. dissertation, Massachusetts Institute of Technology, 1969.

[74] D. T. Herron, "Context, word-class, and prosody in the recognition of openand closed-class words," Ph.D. dissertation, University of California, 1998.

[75] A. Cutler and D. Norris, "The role of strong syllables in segmentation for lexical access," Journal of Experimental Psychology: Human Perception and Performance, vol. 14, pp. 113-121, 1988.

[76] D. H. Klatt, "Linguistic uses of segmental duration in English: Acoustic and perceptual evidence," Journal of the Acoustical Society of America, vol. 59, no. 5, pp. 1208-1221, 1976. 
[77] P. Price, M. Ostendorf, S. Shattuck-Hufnagel, and C. Fong, "The use of prosody in syntactic disambiguation," Journal of the Acoustical Society of America, vol. 90, no. 6, pp. 2956-2965, 1991.

[78] A. Wingfield, "The intonation-syntax interaction: Prosodic features in perceptual processing of sentences," in Structure and Process in Speech Perception, A. Cohen and S. G. Nooteboom, Eds. New York, USA: Springer-Verlag, 1975.

[79] C. M. Beach, "The interpretation of prosodic patterns at points of syntactic structure ambiguity: Evidence for cue trading relations," Journal of Memory and Language, vol. 30, no. 6, pp. 644-663, 1991.

[80] F. Grosjean and C. Hirt, "Using prosody to predict the end of sentences in English and French: Normal and brian-damaged subjects," Language and Cognitive Processes, vol. 11, no. 1/2, pp. 107-134, 1996.

[81] A. Cutler, "Phoneme-monitoring reaction time as a function of preceding intonation contour," Perception and Psychophysics, vol. 20, no. 1, pp. 55-60, 1976.

[82] A. Cutler, "Components of prosodic effects in speech recognition," in Proc. Eleventh International Congress of Phonetic Sciences, Tallinn, Estonia, 1987, pp. 84-87.

[83] J. Terken and J. Hirschberg, "Deaccentuation of words representing "given" information: Effects of persistence of grammatical function and surface position," Language and Speech, vol. 37, pp. 125-145, 1994.

[84] W. van Donselaar and J. Lentz, "The function of sentence accents and given/new information in speech processing: Different strategies for normalhearing and hearing-impaired listeners?" Language and Speech, vol. 37, no. 4, pp. 375-391, 1994.

[85] G. Brown, K. L. Currie, and J. Kenworthy, Questions of Intonation. London, Great Britain: Croom Helm, 1980.

[86] J. Hirschberg and B. Grosz, "Intonational features of local and global discourse structure," in Proc. Workshop on Speech and Natural Language, San Mateo, CA, USA, 1992, pp. 441-446.

[87] R. Carlson, K. Gustafson, and E. Strangert, "Modelling hesitation for synthesis of spontaneous speech," in Proc. Speech Prosody, Dresden, Germany, 2006 . 
[88] C. Nakatani and J. Hirschberg, "A speech-first model for repair detection and correction," in Proc. 31st Annual Meeting of the Association for Computational Linguistics, Columbus, OH, USA, 1993, pp. 46-53.

[89] J. Bear, J. Dowding, and E. Shriberg, "Integrating multiple knowledge sources for detection and correction of repairs in human-computer dialog," in Proc. 30th Annual Meeting of the Association for Computational Linguistics, Newark, NJ, USA, 1992, pp. 56-63.

[90] A. Tickle, "English and Japanese speakers' emotion vocalisation and recognition: A comparison highlighting vowel quality," in ISCA Tutorial and Research Workshop on Speech and Emotion, Newcastle, Northern Ireland, 2000, pp. 104-109.

[91] T. Otake, G. Hatano, and K. Yoneyama, "Speech segmentation by Japanese listeners," in Phonological Structure and Language Processing: CrossLinguistic Studies, T. Otake and A. Cutler, Eds. Berlin, Germany: Mouton de Gruyter, 1996.

[92] T. Otake, G. Hatano, A. Cutler, and J. Mehler, "Mora or syllable? Speech segmentation in Japanese," Journal of Memory and Language, vol. 32, pp. 358-378, 1993.

[93] S. Peppé, J. Maxim, and B. Wells, "Prosodic variation in southern British English," Language and Speech, vol. 43, no. 3, pp. 309-334, 2000.

[94] J. B. Pierrehumbert, "The phonology and phonetics of english intonation," Ph.D. dissertation, Massachusetts Institute of Technology, 1980.

[95] M. Liberman, "The intonational system of english," Ph.D. dissertation, Massachusetts Institute of Technology, 1975.

[96] J. B. Pierrehumbert, "Automatic recognition of intonation patterns," in Proc. 21st Annual Meeting of the Association for Computational Linguistics, Cambridge, MA, USA, 1983, pp. 85-90.

[97] P. A. Taylor, "A phonetic model of english intonation," Ph.D. dissertation, University of Edinburgh, 1992.

[98] C. W. Wightman and M. Ostendorf, "Automatic labeling of prosodic patterns," IEEE Transactions on Speech and Audio Processing, vol. 2, no. 4, pp. 469-481, 1994. 
[99] K. Ross and M. Ostendorf, "Prediction of abstract prosodic labels for speech synthesis," Computer Speech and Language, vol. 10, no. 3, pp. 155-185, 1996.

[100] N. Braunschweiler, "Automatic detection of prosodic cues," Ph.D. dissertation, Universität Konstanz, 2003.

[101] K. Silverman, M. Beckman, J. Pitrelli, M. Ostendorf, C. Wightman, P. Price, J. Pierrehumbert, and J. Hirschberg, "TOBI: A standard for labeling English prosody," in Proc. International Conference on Spoken Language Processing, Banff, Canada, 1992.

[102] M. E. Beckman and G. A. Elam, "Guidelines for ToBI labelling," Ohio State University, Tech. Rep., 1997.

[103] M. Beckman and J. Pierrehumbert, "Intonation structure in Japanese and English," Phonology Yearbook, vol. 3, pp. 255-309, 1986.

[104] J. Hirschberg and M. E. Beckman, "The ToBI annotation conventions," Ohio State University, Tech. Rep., 1994.

[105] C. W. Wightman, "ToBI or not ToBI," in Proc. International Conference on Speech Prosody, Aixen-Provence, France, 2002.

[106] J. Pitrelli, M. Beckman, and J. Hirschberg, "Evaluation of prosodic transcription labeling reliability in the ToBI framework," in Proc. International Conference on Spoken Language Processing, Yokohama, Japan, 1994, pp. 123-126.

[107] A. K. Syrdal and J. McGory, "Inter-transcriber reliability of ToBI prosodic labeling," in Proc. International Conference on Spoken Language Processing, Beijing, China, 2000, pp. 235-238.

[108] A. K. Syrdal, J. Hirschberg, J. McGory, and M. Beckman, "Automatic ToBI prediction and alignment to speed manual labeling of prosody," Speech Communication, vol. 33, no. 1-2, pp. 135-151, 2001.

[109] C. W. Wightman, A. K. Syrdal, G. Stemmer, A. Conkie, and M. Beutnagel, "Perceptually based automatic prosody labeling and prosodically enriched unit selection improve concatenative text-to-speech synthesis," in Proc. International Conference on Spoken Language Processing, Beijing, China, 2000, pp. 71-74. 
[110] A. Batliner, B. Möbius, G. Möhler, A. Schweitzer, and E. Nöth, "Prosodic models, automatic speech understanding, and speech synthesis: Towards the common ground," in Proc. European Conference on Speech Communication and Technology, vol. 4, Aalborg, Denmark, 2001, pp. 2285-2288.

[111] E. Shriberg and A. Stolcke, "Prosody modeling for automatic speech recognition and understanding," in Mathematical Foundations of Speech and Language Processing, ser. The IMA Volumes in Mathematics and its Applications, M. Johnson, S. P. Khudanpur, M. Ostendorf, and R. Rosenfeld, Eds. New York, USA: Springer-Verlag, 2004, vol. 138, pp. 105-114.

[112] W. A. Lea, "Prosodic aids to speech recognition," in Trends in Speech Recognition, W. A. Lea, Ed. Prentice-Hall, Inc., 1980, pp. 166-205.

[113] K. Bishop, "Modeling sentential stress in the context of a large vocabulary continuous speech recognizer," in Proc. International Conference on Spoken Language Processing, Banff, Canada, 1992, pp. 437-440.

[114] K. Chen and M. Hasegawa-Johnson, "How prosody improves word recognition," in Proc. International Conference on Speech Prosody, Nara, Japan, 2004 .

[115] T. Cho, "Effects of prosody on articulation in english," Ph.D. dissertation, University of California at Los Angeles, 2001.

[116] S. E. Levinson, "Continuously variable duration hidden Markov models for speech analysis," in Proc. International Conference on Acoustics, Speech, and Signal Processing, Tokyo, Japan, 1986, pp. 1241-1244.

[117] M. J. Russell and A. E. Cook, "Experimental evaluation of duration modelling techniques for automatic speech recognition," in Proc. International Conference on Acoustics, Speech, and Signal Processing, Dallas, TX, USA, 1987, pp. 2376-2379.

[118] J. Bilmes, "What HMMs can't do: A graphical model perspective"," in Proc. ATR Workshop Beyond HMMs, Kyoto, Japan, 2004.

[119] M. Ostendorf, I. Shafran, and R. Bates, "Prosody models for conversational speech recognition," in Proc. of the 2nd Plenary Meeting and Symposium on Prosody and Speech Processing, 2003, pp. 147-154. 
[120] J. Hirschberg, D. Litman, and M. Swerts, "Prosodic cues to recognition errors," in Proc. of the Automatic Speech Recognition and Understanding Workshop, Keystone, CO, USA, 1999.

[121] N. M. Veilleux and M. Ostendorf, "Prosody/parse scoring and its applications in ATIS," in Proc. ARPA HLT Workshop, Princeton, NJ, USA, 1993, pp. $335-340$.

[122] A. Batliner and E. Nöth, "Prosody and automatic speech recognition - why not yet a success story and where to go from here," in Proc. 2nd Plenary Meeting and Symposium on Prosody and Speech Processing, Tokyo, Japan, 2003, pp. 357-364.

[123] A. Batliner, J. Buckow, R. Huber, V. Warnke, E. Nöth, and H. Niemann, "Prosodic feature evaluation: Brute force or well designed?" in Proc. 14th Int. Congress of Phonetic Sciences, San Francisco, CA, USA, 1999, pp. 23152318 .

[124] V. R. R. Gadde, "Modeling word durations," in Proc. International Conference on Spoken Language Processing, Beijing, China, 2000, pp. 601-604.

[125] A. Stolcke, E. Shriberg, D. Hakkani-Tür, and G. Tür, "Modeling the prosody of hidden events for improved word recognition," in Proc. 6th European Conference on Speech Communication and Technology, Budapest, Hungary, 1999, pp. $307-310$.

[126] K. Chen and M. Hasegawa-Johnson, "Improving the robustness of prosody dependent language modeling based on prosody syntax dependence," in IEEE Workshop on Automatic Speech Recognition and Understanding, St. Thomas, U.S. Virgin Islands, 2003, pp. 435-440.

[127] E. T. Jaynes, "Information theory and statistical mechanics," Physics Review, vol. 106, no. 4, pp. 620-630, 1957.

[128] H. W. Kuhn and A. W. Tucker, "Nonlinear programming," in Proc. Second Berkeley Symposium on Mathematical Statistics and Probability. Berkeley, CA, USA: University of California Press, 1951, pp. 481-490.

[129] S. Kullback, Information Theory and Statistics. New York, USA: Wiley, 1959.

[130] F. Jelinek, Statistical Methods for Speech Recognition. Cambridge, USA: MIT Press, 1998. 
[131] J. Darroch and D. Ratcliff, "Generalized iterative scaling for log-linear models," Annals of Mathematical Statistics, vol. 43, no. 5, pp. 1470-1480, 1972.

[132] S. Della Pietra, V. Della Pietra, and J. Lafferty, "Inducing features of random fields," IEEE Transactions on Pattern Analysis and Machine Intelligence, vol. 19, no. 4, pp. 380-393, 1997.

[133] R. Jin, R. Yan, J. Zhang, and A. Hauptmann, "A faster iterative scaling algorithm for conditional exponential model," in Proc. International Conference on Machine Learning, Washington DC, USA, 2003.

[134] R. Fletcher and C. M. Reeves, "Function minimization by conjugate gradients," The Computer Journal, vol. 7, no. 2, pp. 149-154, 1964.

[135] J. Nocedal, "Updating quasi-newton matrices with limited storage," Mathematics of Computation, vol. 35, no. 151, pp. 773-782, 1980.

[136] R. Malouf, "A comparison of algorithms for maximum entropy parameter estimation," in Proc. Sixth Conference on Natural Language Learning, 2002, pp. $49-55$.

[137] T. P. Minka, "A comparison of numerical optimizers for logistic regression," http://research.microsoft.com/ minka/papers/logreg/minka-logreg.pdf, 2003.

[138] A. Ratnaparkhi, "Maximum entropy models for natural language ambiguity resolution," Ph.D. dissertation, University of Pennsylvania, 1998.

[139] A. L. Berger, S. A. Della Pietra, and V. J. Della Pietra, "A maximum entropy approach to natural language processing," Computational Linguistics, vol. 22, no. 1, pp. 39-72, 1996.

[140] S. F. Chen and R. Rosenfeld, "A Gaussian prior for smoothing maximum entropy models," Carnegie Mellon University, Tech. Rep., 1999.

[141] J. Kazama and J. Tsujii, "Evaluation and extension of maximum entropy models with inequality constraints," in Proc. Conference on Empirical Methods in Natural Language Processing, Sapporo, Japan, 2003, pp. 137-144.

[142] J. Goodman, "Exponential priors for maximum entropy models," in Proc. Annual Meeting of the Association for Computational Linguistics, 2004. 
[143] S. F. Chen and J. T. Goodman, "An empirical study of smoothing techniques for language modeling," in Proc. 34th Meeting of the Association for Computational Linguistics, Santa Cruz, CA, USA, 1996, pp. 310-318.

[144] R. Rosenfeld, "Adaptive statistical language modeling: A maximum entropy approach," Ph.D. dissertation, Carnegie Mellon University, 1994.

[145] M. Ostendorf, P. Price, and S. Shattuck-Hufnagel, "The Boston University Radio News Corpus," Boston University, Tech. Rep., 1995.

[146] M. Marcus and B. Santorini, "Building a very large natural language corpora: The Penn Treebank," Computational Linguistics, vol. 19, no. 2, pp. 313-330, 1993.

[147] M. Meteer, R. Schwartz, and R. Weischedel, "POST: Using probabilities in language processing," in Proc. International Joint Conference on Artifical Intelligence, Sydney, Australia, 1991, pp. 960-965.

[148] M. Hasegawa-Johnson, K. Chen, J. Cole, S. Borys, S. Kim, A. Cohen, T. Zhang, J. Choi, H. Kim, T. Yoon, and S. Chavarria, "Simultaneous recognition of words and prosody in the Boston University radio speech corpus," Speech Communication, vol. 46, no. 3-4, pp. 418-439, 2005.

[149] A. Stolcke, "SRILM - an extensible language modeling toolkit," in Proc. International Conference on Spoken Language Processing, Denver, CO, USA, 2002, pp. 901-904.

[150] S. J. Benson and J. J. Moré, "A limited memory variable metric for bound constrained minimization," Mathematics and Computer Science Division, Argonne National Laboratory, Tech. Rep., 2001.

[151] S. J. Young, "The HTK hidden Markov model toolkit: Design and philosophy," Cambridge University Engineering Department, Tech. Rep., 1994.

[152] F. J. Och, N. Ueffing, and H. Ney, "An efficient A* search algorithm for statistical machine translation," in Proc. Workshop on Data-Driven Methods in Machine Translation, Toulouse, France, 2001, pp. 1-8.

[153] L. Nguyen, R. Schwartz, Y. Zhao, and G. Zavaliagkos, "Is N-best dead?" in Proc. Workshop on Human Language Technology, Plainsboro, NJ, USA, 1994, pp. $411-414$. 
[154] P. Taylor, A. W. Black, and R. Caley, "The architecture of the Festival speech synthesis system," in Proc. Third ESCA Workshop in Speech Synthesis, Jenolan Caves, Australia, 1998, pp. 147-151.

[155] D. H. Milone and A. J. Rubio, "Prosodic and accentual information for automatic speech recognition," IEEE Transactions on Speech and Audio Processing, vol. 11, no. 4, pp. 321-333, 2003.

[156] A. Batliner, E. Nöth, J. Buckow, R. Huber, V. Warnke, and H. Niemann, "Duration features in prosodic classification: Why normalization comes second, and what they really encode," in Proc. ISCA Tutorial and Research Workshop on Speech recognition and Understanding, Red Bank, NJ, USA, 2001, pp. 23-28.

[157] C. W. Wightman, S. Shattuck-Hufnagel, M. Ostendorf, and P. J. Price, "Segmental durations in the vicinity of prosodic phrase boundaries," Journal of the Acoustical Society of America, vol. 91, no. 3, pp. 1707-1717, 1992.

[158] P. Taylor, "Analysis and synthesis of intonation using the Tilt model," Journal of the Acoustical Society of America, vol. 107, no. 3, pp. 1697-1714, 2000.

[159] R. Herman, M. Beckman, and K. Honda, "Subglottal pressure and final lowering in English," in Proc. International Conference on Spoken Language Processing, Philadelphia, PA, USA, 1996.

[160] D. Klakow, "Log-linear interpolation of language models," in Proc. International Conference on Spoken Language Processing, Sydney, Australia, 1998, pp. 1695-1698.

[161] V. Sandrini, M. Federico, and M. Cettolo, "Maximum entropy tagging with binary and real-valued features," in Proc. Workshop on Learning Structured Information in Natural Language Applications, 11th Conference of the European Chapter of the Association for Computational Linguistics, Trento, Italy, 2006, pp. 1-8.

[162] C. White, J. Droppo, A. Acero, and J. Odell, "Maximum entropy confidence estimation for speech recognition," in Proc. International Conference on Acoustics, Speech, and Signal Processing, Honolulu, Hawaii, USA, 2007, pp. 809-812.

[163] L. J. P. van der Maaten, E. O. Postma, and H. J. van den Henrik, "Dimensionality reduction: A comparative review," Maastricht University, Tech. 
Rep., 2007, submitted to IEEE Transactions on Pattern Analysis and Machine Intelligence.

[164] X. He and P. Niyogi, "Locality preserving projections," in Advances in Neural Information Processing Systems 16, S. Thrun, L. Saul, and B. Schölkopf, Eds. Cambridge, USA: MIT Press, 2003.

[165] F. R. K. Chung, Spectral Graph Theory. American Mathematical Society, 1997.

[166] M. Belkin and P. Niyogi, "Laplacian eigenmaps and spectral techniques for embedding and clustering," Advances in Neural Information Processing Systems, vol. 14, pp. 585-591, 2002.

[167] D. Cai, X. He, and J. Han, "Document clustering using locality preserving indexing," IEEE Transactions on Knowledge and Data Engineering, vol. 17, no. 12, pp. 1624-1637, 2005.

[168] J. G. Kahn, M. Lease, E. Charniak, M. Johnson, and M. Ostendorf, "Effective use of prosody in parsing conversational speech," in Proc. Conference on Empirical Methods in Natural Language Processing, Vancouver, Canada, 2005, pp. 233-240.

[169] A. Varga, H. J. M. Steeneken, M. Tomlinson, and D. Jones, "The NOISEX-92 study on the effect of additive noise on automatic speech recognition," DRA Speech Research Unit, Tech. Rep., 1992.

[170] H. Hirsch, "Filtering and noise adding tool," http://dnt.kr.hsniederrhein.de/download.html.

[171] D. R. Campbell, K. J. Palomäki, and G. J. Brown, "Roomsim, a MATLAB simulation of "shoebox" room acoustics for use in teaching and research," Computing and Information Systems, vol. 9, no. 3, pp. 48-51, 2005. 